\title{
Theoretical investigation of energy levels and transition data for S II, Cl III, Ar IV $\star$
}

\author{
P. Rynkun ${ }^{1}$, G. Gaigalas ${ }^{1}$, and P. Jönsson ${ }^{2}$ \\ ${ }^{1}$ Institute of Theoretical Physics and Astronomy, Vilnius University, Sauletekio av. 3, 10222 Vilnius, Lithuania \\ e-mail: pavel.rynkun@tfai.vu.lt \\ ${ }^{2}$ Group for Materials Science and Applied Mathematics, Malmö University, 20506 Malmö, Sweden
}

Received 20 December 2018 / Accepted 28 January 2019

\begin{abstract}
Aims. The aim of this work is to present accurate and extensive results of energy spectra and transition data for the S II, Cl III, and Ar IV ions. These data are useful for understanding and probing physical processes and conditions in various types of astrophysical plasmas.

Methods. The multiconfiguration Dirac-Hartree-Fock (MCDHF) and relativistic configuration interaction (RCI) methods, which are implemented in the general-purpose relativistic atomic structure package GRASP2K, are used in the present work. In the RCI calculations the transverse-photon (Breit) interaction, the vacuum polarization, and the self-energy corrections are included.

Results. Energy spectra are presented comprising the 134, 87, and 103 lowest states in S II, Cl III, and Ar IV, respectively. Energy levels are in very good agreement with NIST database recommended values and associated with smaller uncertainties than energies from other theoretical computations. Electric dipole (E1), magnetic dipole (M1), and electric quadrupole (E2) transition data are computed between the above states together with the corresponding lifetimes. Based on internal validation, transition rates for the majority of the stronger transitions are estimated to have uncertainties of less than $3 \%$.
\end{abstract}

Key words. atomic data

\section{Introduction}

Atomic data are of importance in the understanding of the physical processes and conditions in various types of astrophysical plasmas, for example in the determination of chemical abundances of the elements and in the estimation of radiative transfer through stellar plasmas. The quality of atomic data have a large impact on the accuracy of chemical abundances (Juan de Dios \& Rodríguez 2017; Kisielius et al. 2014). Juan de Dios \& Rodríguez (2017) computed the ionic abundances of O II, O III, N II, Cl III, Ar III, Ar IV, Ne III, S II, and $\mathrm{S}$ III, and the total abundances of the corresponding elements. They observed that data for some of the studied ions need to be improved in order to derive more reliable values of chemical abundances in high-density nebulae.

The studied elements (S II, Cl III, Ar IV) are in the astrophysically important group of low-ionization ions which belongs to the phosphorous isoelectronic sequence. Fritzsche et al. (1999) used the multiconfiguration Dirac-Fock method to study forbidden transitions in the $3 \mathrm{~s}^{2} 3 \mathrm{p}^{3}$ configuration for phosphorus-like ions with low nuclear charge $Z$. Fischer et al. (2006) computed energy levels, lifetimes, and transition data for the sodium-like to argon-like sequences using the multiconfiguration Hartree-Fock (MCHF) and multiconfiguration Dirac-Hartree-Fock (MCDHF) methods.

\footnotetext{
* Tables 5-7 are only available at the CDS via anonymous ftp to cdsarc.u-strasbg.fr $(130.79 .128 .5)$ or via http://cdsarc. u-strasbg.fr/viz-bin/qcat?J/A+A/623/A155
}

Tayal \& Zatsarinny (2010) used the B-spline Breit-Pauli Rmatrix method to compute energy levels of the $3 s^{2} 3 p^{3}, 3 s 3 p^{4}$, $3 s^{2} 3 p^{2} 3 d, 3 s^{2} 3 p^{2} 4 s$, and $3 s^{2} 3 p^{2} 4 p$ configurations and transitions in S II. Kisielius et al. (2014) presented the energy levels and transition data for S II using HF and quasirelativistic (QR) methods with transformed radial orbitals.

Sossah \& Tayal (2012) used the MCHF and B-spline BreitPauli R-matrix method to calculate transition probabilities and effective collision strengths for Cl III. Schectman et al. (2005) measured lifetimes and branching fractions with beam-foil techniques and derived oscillator strengths for transitions in $\mathrm{Cl}$ II and III.

Bredice et al. (1995) observed the spectra of Ar IV in the 280-5000 A wavelength range and reanalyzed the $3 \mathrm{~s}^{2} 3 \mathrm{p}^{3}, 3 \mathrm{~s} 3 \mathrm{p}^{4}$, $3 s^{2} 3 p^{2}(3 d+4 s)$ configurations. Djenize \& Bukvić (2001) and Burger et al. (2012) studied transition probabilities in Ar III and Ar IV ions. Raineri et al. (2018) used a pulsed discharge light source to study the spectrum of Ar III and Ar IV in the 480$6218 \AA$ region and predicted new levels using the Cowan code (Cowan 1981).

In this work energy spectrum calculations were performed for the 134 (72 even and 62 odd), 87 (52 even and 35 odd), and 103 (44 even and 59 odd) lowest states in the S II, Cl III, and Ar IV ions, respectively. Electric dipole, magnetic dipole, and electric quadrupole transition data were computed along with the corresponding lifetimes of these states. The calculations were done using the general-purpose relativistic atomic structure package GRASP2K (Jönsson et al. 2013) with the modifications that are included in the newest version of the GRASP2018 package (Fischer et al. 2019). 
Table 1. Summary of active space construction.

\begin{tabular}{|c|c|c|c|c|}
\hline \multicolumn{2}{|c|}{ MR set } & \multirow[t]{2}{*}{ Active space } & \multicolumn{2}{|c|}{$N_{\mathrm{CSFs}}$} \\
\hline Even & Odd & & Even & Odd \\
\hline & & $\begin{array}{l}\text { S II } \\
\text { RCI }\end{array}$ & & \\
\hline $\begin{array}{c}3 \mathrm{~s} 3 \mathrm{p}^{4}, 3 \mathrm{~s}^{2} 3 \mathrm{p}^{2} 3 \mathrm{~d} \\
3 \mathrm{~s}^{2} 3 \mathrm{p}^{2} 4 \mathrm{~s}, 3 \mathrm{~s}^{2} 3 \mathrm{p}^{2} 4 \mathrm{~d} \\
3 \mathrm{~s}^{2} 3 \mathrm{p}^{2} 5 \mathrm{~s}\end{array}$ & $\begin{array}{c}3 \mathrm{~s}^{2} 3 \mathrm{p}^{3}, 3 \mathrm{~s}^{2} 3 \mathrm{p}^{2} 4 \mathrm{p} \\
3 \mathrm{~s} 3 \mathrm{p}^{3} 3 \mathrm{~d}, 3 \mathrm{~s}^{2} 3 \mathrm{p}^{2} 4 \mathrm{f} \\
3 \mathrm{~s}^{2} 3 \mathrm{p}^{2} 5 \mathrm{p} \\
\text { RCI }(\mathrm{CV}) \text { additi }\end{array}$ & $\begin{array}{l}\{9 \mathrm{~s}, 9 \mathrm{p}, 9 \mathrm{~d}, 8 \mathrm{f}, 8 \mathrm{~g}, 8 \mathrm{~h}, 8 \mathrm{i}\} \\
\text { ally included configurations }\end{array}$ & 478170 & 873674 \\
\hline $\begin{array}{c}3 \mathrm{~s}^{2} 3 \mathrm{p}^{2} 5 \mathrm{~d}, 3 \mathrm{p}^{4} 3 \mathrm{~d} \\
3 \mathrm{~s} 3 \mathrm{p}^{2} 3 \mathrm{~d}^{2}, 3 \mathrm{p}^{4} 4 \mathrm{~s} \\
3 \mathrm{p}^{4} 4 \mathrm{~d}, 3 \mathrm{~s} 3 \mathrm{p}^{2} 3 \mathrm{~d} 4 \mathrm{~d} \\
3 \mathrm{~s}^{2} \mathrm{p}^{2} 3 \mathrm{~d} 4 \mathrm{~s}, 3 \mathrm{p}^{4} 5 \mathrm{~s} \\
3 \mathrm{~s} 3 \mathrm{p}^{2} 3 \mathrm{~d} 5 \mathrm{~s}\end{array}$ & $\begin{array}{c}3 p^{5}, 3 s 3 p^{2} 3 d 4 p \\
3 s 3 p^{2} 3 d 5 p, 3 p^{4} 4 p \\
3 p^{4} 5 p, 3 p^{4} 4 f \\
3 s 3 p^{2} 3 d 4 f, 3 s 3 p^{3} 5 d \\
3 s 3 p^{3} 4 d, 3 s 3 p^{3} 4 s\end{array}$ & $\begin{array}{c}\{9 \mathrm{~s}, 9 \mathrm{p}, 9 \mathrm{~d}, 8 \mathrm{f}, 8 \mathrm{~g}, 8 \mathrm{~h}, 8 \mathrm{i}\} \\
\{6 \mathrm{~s}, 6 \mathrm{p}, 5 \mathrm{~d}, 5 \mathrm{f}\} \text { for } S \text { from } 2 \mathrm{p}\end{array}$ & 6220422 & 9540812 \\
\hline & & $\begin{array}{l}\text { CI III } \\
\text { RCI }\end{array}$ & & \\
\hline $\begin{array}{c}3 \mathrm{~s} 3 \mathrm{p}^{4}, 3 \mathrm{~s}^{2} 3 \mathrm{p}^{2} 3 \mathrm{~d} \\
3 \mathrm{~s}^{2} 3 \mathrm{p}^{2} 4 \mathrm{~s}, 3 \mathrm{~s}^{2} 3 \mathrm{p}^{2} 4 \mathrm{~d}\end{array}$ & $\begin{array}{c}3 s^{2} 3 p^{3}, 3 s^{2} 3 p^{2} 4 p \\
3 s 3 p^{3} 3 d, 3 p^{5} \\
\text { RCI }(C V) \text { additic }\end{array}$ & $\begin{array}{l}\qquad 9 \mathrm{~s}, 9 \mathrm{p}, 9 \mathrm{~d}, 8 \mathrm{f}, 8 \mathrm{~g}, 8 \mathrm{~h}, 8 \mathrm{i}\} \\
\text { ally included configurations }\end{array}$ & 492140 & 583117 \\
\hline $\begin{array}{c}3 p^{4} 3 d, 3 s 3 p^{2} 3 d^{2} \\
3 s 3 p^{2} 3 d 4 s, 3 s 3 p^{2} 3 d 4 d\end{array}$ & $\begin{array}{l}3 s^{2} 3 p 3 d^{2}, 3 s 3 p^{3} 4 s \\
3 p^{4} 4 p, 3 s 3 p^{2} 3 d 4 p\end{array}$ & $\begin{array}{c}\{9 \mathrm{~s}, 9 \mathrm{p}, 9 \mathrm{~d}, 8 \mathrm{f}, 8 \mathrm{~g}, 8 \mathrm{~h}, 8 \mathrm{i}\} \\
\{6 \mathrm{~s}, 6 \mathrm{p}, 6 \mathrm{~d}, 5 \mathrm{f}\} \text { for } S \text { from } 2 \mathrm{p}\end{array}$ & 6466816 & 4111005 \\
\hline & & $\begin{array}{l}\text { Ar IV } \\
\text { RCI }\end{array}$ & & \\
\hline $\begin{array}{c}3 \mathrm{~s} 3 \mathrm{p}^{4}, 3 \mathrm{~s}^{2} 3 \mathrm{p}^{2} 3 \mathrm{~d} \\
3 \mathrm{~s}^{2} 3 \mathrm{p}^{2} 4 \mathrm{~s}\end{array}$ & $\begin{array}{c}3 s^{2} 3 p^{3}, 3 s^{2} 3 p^{2} 4 p \\
3 s 3 p^{3} 3 d, 3 p^{5} \\
\text { RCI }(C V) \text { additic }\end{array}$ & $\begin{array}{l}\qquad\{\mathrm{s}, 8 \mathrm{p}, 8 \mathrm{~d}, 8 \mathrm{f}, 8 \mathrm{~g}, 7 \mathrm{~h}, 7 \mathrm{i}\} \\
\text { ally included configurations }\end{array}$ & 224651 & 431584 \\
\hline $\begin{array}{c}3 s^{2} 3 p^{2} 4 d, 3 p^{4} 3 d \\
3 s 3 p^{2} 3 d^{2}, 3 s 3 p^{2} 3 d 4 s \\
3 s 3 d^{2} 4 s, 3 s^{2} 3 d^{3}\end{array}$ & $\begin{array}{l}3 s^{2} 3 p 3 d^{2}, 3 s 3 p^{3} 4 s \\
3 p^{4} 4 p, 3 s 3 p^{2} 3 d 4 p \\
3 p^{3} 3 d^{2}, 3 s^{2} 3 p^{2} 4 f\end{array}$ & $\begin{array}{c}\{9 \mathrm{~s}, 8 \mathrm{p}, 8 \mathrm{~d}, 8 \mathrm{f}, 8 \mathrm{~g}, 7 \mathrm{~h}, 7 \mathrm{i}\} \\
\{7 \mathrm{~s}, 6 \mathrm{p}, 6 \mathrm{~d}, 6 \mathrm{f}, 5 \mathrm{~g}\} \text { for } \mathrm{S} \text { from } 2 \mathrm{p}\end{array}$ & 4946496 & 7329546 \\
\hline
\end{tabular}

Notes. RCI denotes CSF expansions from SD substitutions from the valence electrons of the configurations in the MR. RCI(CV) denotes CSF expansions from SD substitutions from the valence electrons of the configurations in the extended MR along with CSFs obtained by allowing at most one substitution (S) from the $2 p$ shell to a smaller active orbital space. $N_{\mathrm{CSFs}}$ is the number of CSFs.

\section{Method}

\subsection{Computational procedure}

The GRASP2K package used for the computations is based on the MCDHF and relativistic configuration interaction (RCI) methods. More details about these approaches can be found in Fischer et al. (2016) and Grant (2007).

In the MCDHF approximation atomic state functions (ASFs) are given as linear combinations of symmetry adapted configuration state functions (CSFs)

$\Psi(\gamma P J M)=\sum_{i=1}^{N_{\mathrm{CSFs}}} c_{i} \Phi\left(\gamma_{i} P J M\right)$,

where $J$ and $M$ are the angular quantum numbers and $P$ is parity. The CSFs $\Phi\left(\gamma_{i} P J M\right)$ are built from products of one-electron Dirac orbitals. In the relativistic self-consistent field procedure both the radial parts of the Dirac orbitals and the expansion coefficients were optimized to self-consistency.

In RCI computations the wave function is expanded in CSFs and only the expansion coefficients are determined by diagonalizing the Hamiltonian matrix. The RCI method was used to include the transverse-photon (Breit) interaction and quantum electrodynamic (QED) corrections: the vacuum polarization and the self-energy.

In this work ASFs were obtained as expansions over $j j$ coupled CSFs. To transform these ASFs into an $L S J$-coupled
CSF basis the method provided by Gaigalas et al. (2003, 2017) was used.

\subsection{Computational scheme}

For all three ions (S II, Cl III, Ar IV) similar computational schemes were used. As a starting point, MCDHF calculations were performed in the extended optimal level (EOL) scheme (Dyall et al. 1989) for the weighted average of the even and odd parity states. For the construction of the ASFs the multireference-single-double (MR-SD) method (Fischer et al. 2016) was used. In this approach the CSF expansions were obtained by allowing SD substitutions from the configurations in the MR to active orbital sets. Only CSFs that have non-zero matrix elements with the CSFs belonging to the configurations in the MR were retained. No substitutions were allowed from the $1 \mathrm{~s}, 2 \mathrm{~s}, 2 \mathrm{p}$ shells, which define an inactive closed core. The MR and the active orbital sets for each of the ions are presented in Table 1. The MCDHF calculations were followed by RCI calculations, including the Breit interaction and leading QED effects. The RCI calculations were done separately for even and odd states.

At the last step, referred to as the $\mathrm{RCI}(\mathrm{CV})$ step in the tables, the MR was extended to include additional important configurations, and core-valence $(\mathrm{CV})$ correlation effects were accounted for by allowing at most one substitution also from the $2 p$ shell. The substitutions from the $2 p$ shell increase the 
number of CSFs dramatically and for this reason these substitutions were restricted to a smaller orbital set.

The large-scale calculations were performed with the MPI version of the GRASP code.

\section{Results}

The accuracy of the wave functions from the present calculation and some previous calculations was evaluated by comparing calculated energy levels with data from the NIST database (Kramida et al. 2018). In Table 2 a summary of this evaluation is presented: the number of computed energy levels (No. of levels in Ref.) and the average percentage difference between NIST and the different methods for the states covered by these methods (Av. difference).

The inclusion of the CV electron correlations and the extension of the MR set in the calculations improve the results. As is seen from Table 2 the averaged difference of the computed energy spectra (final $\mathrm{RCI}(\mathrm{CV})$ results) relative to the energies from the NIST database is $0.22 \%, 0.18 \%$, and $0.21 \%$, respectively, for the S II, Cl III, and Ar IV ions. Comparing the present results with results from other theoretical computations we obtain a better agreement with values given in the NIST database, except for the S II ion. The averaged uncertainties of energies presented in Tayal \& Zatsarinny (2010) is only $0.06 \%$, but they cover fewer energy levels. For the first time, levels of the $3 \mathrm{~s} 3 \mathrm{p}^{3} 3 d$ configuration are presented for the S II, Cl III, and Ar IV ions.

The mean contribution of the Breit and QED corrections to the final results is $0.05 \%$ for the studied ions. For the separate state the contribution of these effects can reach $0.1 \%$.

The uncertainty of electric transition data was evaluated based on the quantity $\mathrm{d} T$ (Ekman et al. 2014), which is defined as

$\mathrm{d} T=\frac{\left|A_{l}-A_{v}\right|}{\max \left(A_{l}, A_{v}\right)}$.

Here, $A_{l}$ and $A_{v}$ are transition rates in length and velocity forms. The mean $\mathrm{d} T$ for all presented E1 transitions is $12.00 \%, 5.95 \%$, and $6.47 \%$, respectively for the S II, Cl III, and Ar IV ions. The results for the different ions is discussed in more detail below.

\section{1. $S$ II}

In Table A.1 energy spectra and wave function composition in $L S$-coupling for 72 even states of the $3 \mathrm{~s}^{3} \mathrm{p}^{4}, 3 \mathrm{~s}^{2} 3 \mathrm{p}^{2} 3 \mathrm{~d}, 3 \mathrm{~s}^{2} 3 \mathrm{p}^{2} 4 \mathrm{~d}$, $3 s^{2} 3 p^{2} 4 s, 3 s^{2} 3 p^{2} 5 s$ configurations and for 62 odd states of the $3 s^{2} 3 p^{3}, 3 s^{2} 3 p^{2} 4 p, 3 s 3 p^{3} 3 d, 3 s^{2} 3 p^{2} 4 f, 3 s^{2} 3 p^{2} 5 p$ configurations for S II are given. The states are given with unique labels (Gaigalas et al. 2017). The contribution was marked in bold for the states in which the labels were not assigned with largest contribution to the composition. In Table A.1 lifetimes in length and velocity gauges are also presented.

In Fig. 1 energy levels computed in this work and other theoretical calculations are compared with data from NIST (Kramida et al. 2018). From the figure we see that the relative uncertainties of energy levels obtained in this work in most cases are about $0.2 \%$. Only for levels of the ground configuration the disagreements are larger, about $1.8 \%$.

Transition data such as wavelengths; weighted oscillator strengths; transition rates of E1, M1, and E2 transitions; and the accuracy indicator $\mathrm{d} T$ are given in Table 5, and are available at the CDS. Generally, the uncertainty of transition data is small for
Table 2. Comparison of computed energy levels in the present work and other theoretical results with data from the NIST database for the S II, $\mathrm{Cl}$ III, and Ar IV ions.

\begin{tabular}{|c|c|c|}
\hline Av. difference (in \%) & No. of levels in Ref. & Ref. \\
\hline \multicolumn{3}{|c|}{ S II } \\
\hline 0.34 & $134(126)$ & RCI \\
\hline 0.22 & $134(126)$ & $\mathrm{RCI}(\mathrm{CV})$ \\
\hline 0.48 & $67(67)$ & 1 \\
\hline 0.06 & $70(70)$ & 2 \\
\hline 1.12 & $49(49)$ & $3 a$ \\
\hline 1.18 & $49(49)$ & $3 \mathrm{~b}$ \\
\hline \multicolumn{3}{|c|}{ Cl III } \\
\hline 0.38 & $87(63)$ & $\mathrm{RCI}$ \\
\hline 0.18 & $87(63)$ & $\mathrm{RCI}(\mathrm{CV})$ \\
\hline 0.55 & $67(54)$ & 1 \\
\hline 1.01 & $68(51)$ & $4 \mathrm{a}$ \\
\hline 1.81 & $68(51)$ & $4 \mathrm{~b}$ \\
\hline \multicolumn{3}{|c|}{ Ar IV } \\
\hline 0.47 & $103(56)$ & $\mathrm{RCI}$ \\
\hline 0.21 & $103(56)$ & $\mathrm{RCI}(\mathrm{CV})$ \\
\hline 0.60 & $62(51)$ & 1 \\
\hline
\end{tabular}

Notes. The numbers in parentheses show the number of levels compared with the NIST database.

References. (1) Fischer et al. (2006); (2) Tayal \& Zatsarinny (2010); (3) Kisielius et al. (2014) (a - HF data, b - quasirelativistic data); (4) Sossah \& Tayal (2012) (a - MCHF calculations with 2893 configurations, $\mathrm{b}$ - MCHF calculations with 436 configurations).

the stronger transitions. To display this a scatterplot of $\mathrm{d} T$ versus the transition rate $A$ for computed $\mathrm{E} 1$ transitions is given in Fig. 2. For most of the transitions, $\mathrm{d} T$ is well below $10 \%$, and for the strongest ones $\mathrm{d} T$ is well below $3 \%$. The weak transitions are either intercombination transitions, where in relativistic calculations the low rates result from strong cancellation of several large contributions to the transition moment (Ynnerman \& Fischer 1995), or two-electron one-photon (TEOP) transitions, where the rate is identically zero in the simplest approximation of the wave function and where the transition results from inclusion of correlation effects ( $\mathrm{Li}$ et al. 2010). These types of transitions are still extremely challenging for theory and improved methodology is needed to further decrease the uncertainties.

\section{2. $\mathrm{Cl}$ III}

In Table A. 2 energy spectra, lifetimes, and wave function composition in $L S$-coupling are presented for 52 even states of the $3 \mathrm{~s}^{4} \mathrm{p}^{4}, 3 \mathrm{~s}^{2} 3 \mathrm{p}^{2} 3 \mathrm{~d}, 3 \mathrm{~s}^{2} 3 \mathrm{p}^{2} 4 \mathrm{~s}, 3 \mathrm{~s}^{2} 3 \mathrm{p}^{2} 4 \mathrm{~d}$ configurations and for 35 odd states of the $3 s^{2} 3 p^{3}, 3 s^{2} 3 p^{2} 4 p, 3 s 3 p^{3} 3 d, 3 p^{5}$ configurations in the $\mathrm{Cl}$ III ion. Energy levels are compared with results from NIST (Kramida et al. 2018). In the NIST database some levels of the $3 s^{2} 3 p^{2} 4 p, 3 s^{2} 3 p^{2} 4 d$, and $3 p^{5}$ configurations are flagged with question marks. Comparing these levels with present calculations and other computations there is a good agreement, except for states of the $3 \mathrm{p}^{5}$ configuration. Our energies for states of the $3 \mathrm{p}^{5}$ configuration are lower by $60000 \mathrm{~cm}^{-1}$. These levels in NIST are marked as levels that were determined by interpolation or extrapolation of known experimental values or by semiempirical calculations.

The uncertainty of the computed energy levels comparing with NIST data is less than $0.5 \%$, and in most cases about $0.1 \%$. Only for the first excited levels is the disagreement more than $1 \%$. The averaged uncertainty of computed energy spectra 



Fig. 1. Comparison of computed energy levels and other theoretical calculations with data from the NIST database for S II. The solid lines indicate $0.5 \%$ and the dashed lines $0.2 \%$ deviation from the NIST data. (Kisielius et al. 2014) a - HF data. (Kisielius et al. 2014) b - quasirelativistic data.
Fig. 2. Scatterplot of $\mathrm{d} T$ vs. the transition rate $A$ of E1 transitions for S II. The solid lines indicate the $4 \%$ and $10 \%$ deviations. comparing with NIST data is $0.18 \%$ (Table 2). The present energies are in better agreement with NIST than energies from previous theoretical computations. In addition, in this work more energy levels were studied, and for the first time levels of the $3 \mathrm{~s} 3 \mathrm{p}^{3} 3 \mathrm{~d}$ configuration are presented.

Transition data for E1, M1, and E2 transitions are given in Table 6, and are available at the CDS. In Fig. 3 the scat- terplot of $\mathrm{d} T$ versus the transition rate $A$ is displayed for all presented E1 transitions. The mean $\mathrm{d} T$ for the transitions is $5.95 \%$. For most of the strongest transitions, $\mathrm{d} T$ is well below $2 \%$. Table 3 gives the comparison of the theoretical and experimental results of wavelengths and oscillator strengths for the $3 \mathrm{~s}^{2} 3 \mathrm{p}^{3}\left({ }_{3}^{4} \mathrm{~S}\right){ }^{4} \mathrm{~S}_{3 / 2}^{o} \rightarrow 3 \mathrm{~s} 3 \mathrm{p}^{4}\left({ }_{2}^{3} \mathrm{P}\right){ }^{4} \mathrm{P}_{5 / 2,3 / 2,1 / 2}$ transitions in $\mathrm{Cl}$ III. From the table we see a very good agreement of 


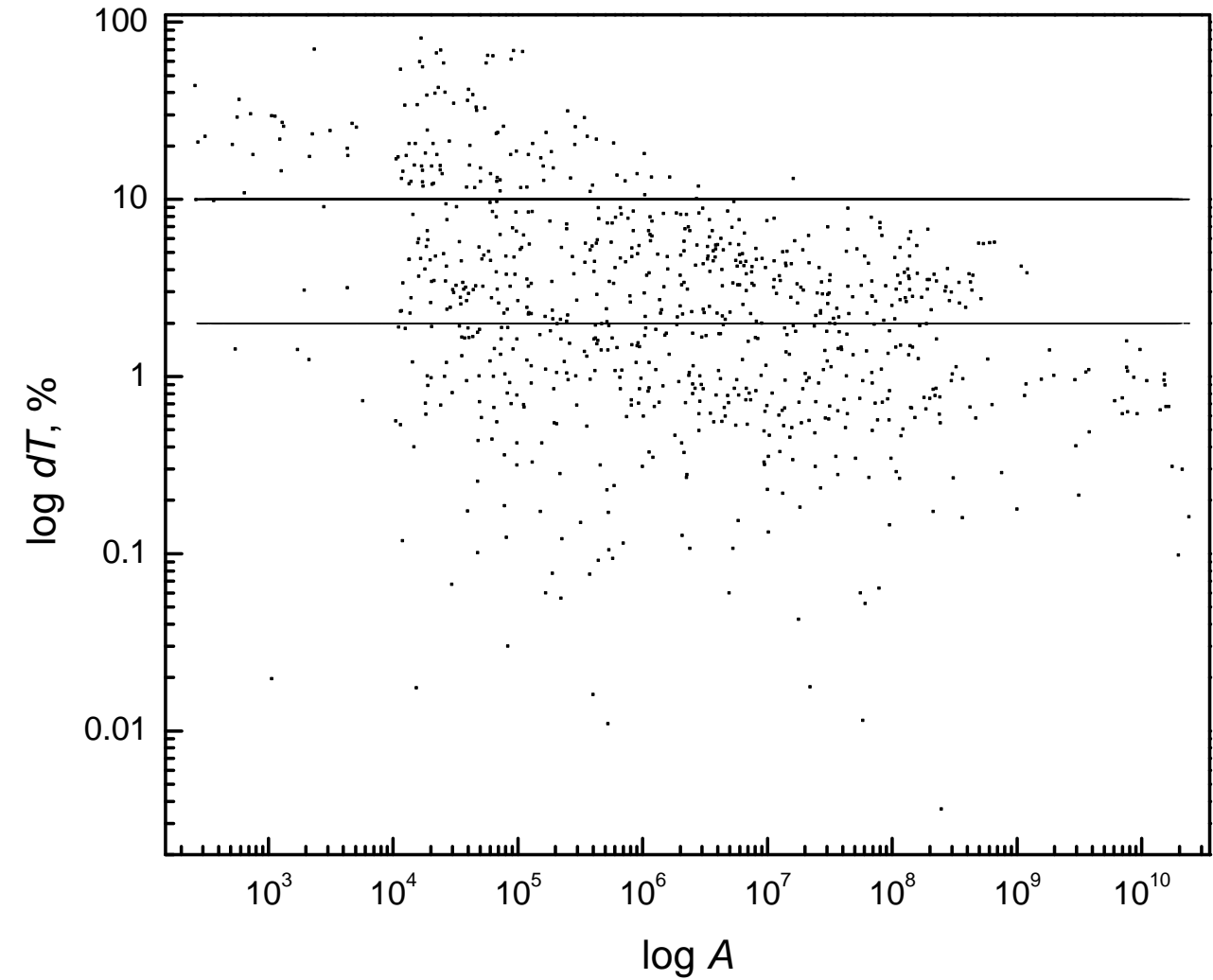

Fig. 3. Scatterplot of $\mathrm{d} T$ vs. the transition rate $A$ of $\mathrm{E} 1$ transitions for $\mathrm{Cl}$ III. The solid lines indicate the $2 \%$ and $10 \%$ deviations.
Table 3. Comparison of wavelengths and oscillator strengths for the $3 \mathrm{~s}^{2} 3 \mathrm{p}^{3}\left({ }_{3}^{4} \mathrm{~S}\right){ }^{4} \mathrm{~S}_{3 / 2}^{o} \rightarrow 3 \mathrm{~s} 3 \mathrm{p}^{4}\left({ }_{2}^{3} \mathrm{P}\right){ }^{4} \mathrm{P}_{5 / 2,3 / 2,1 / 2}$ transitions in $\mathrm{Cl}$ III.

\begin{tabular}{lccc}
\hline \hline \multicolumn{3}{c}{$J_{i} \rightarrow J_{f}$} & Ref. \\
\cline { 1 - 2 } $3 / 2 \rightarrow 5 / 2$ & $3 / 2 \rightarrow 3 / 2$ & $3 / 2 \rightarrow 1 / 2$ & \\
\cline { 1 - 2 } & $\lambda($ in $\AA$ ) \\
\hline 1015.02 & 1008.78 & 1005.28 & 1 (Exp.) \\
1019.22 & 1013.05 & 1009.21 & RCI (This work) \\
1015.14 & 1008.94 & 1005.41 & RCI(CV) (This work) \\
1023.21 & 1017.96 & 1015.04 & 2 \\
1024.48 & 1018.74 & 1015.46 & 3 \\
\hline \multicolumn{3}{c}{$f \times 10^{-2}$} & \\
\hline $2.85 \pm 0.11$ & $1.93 \pm 0.08$ & $0.96 \pm 0.05$ & 4 (Exp.) \\
$3.14(2.6)$ & $2.10(2.9)$ & $1.04(3.8)$ & RCI (This work) \\
$3.10(4.0)$ & $2.07(3.7)$ & $1.04(3.6)$ & RCI(CV) (This work) \\
3.23 & 2.15 & 1.07 & 2 \\
3.21 & 2.14 & 1.07 & 3 \\
\hline
\end{tabular}

Notes. The oscillator strengths are given in the length gauge. The estimated uncertainty in percentage of the oscillator strengths are given in parentheses.

References. (1) Kramida et al. (2018); (2) Fischer et al. (2006); (3) Sossah \& Tayal (2012); (4) Schectman et al. (2005).

wavelengths with the experimental values. Oscillator strengths are a little too large compared with experiment (Schectman et al. 2005).

\section{3. $\operatorname{ArIV}$}

Table A.3 displays energy spectra, lifetimes, and wave function composition in $L S$-coupling for 44 even states of the $3 \mathrm{~s} 3 \mathrm{p}^{4}$,
$3 \mathrm{~s}^{2} 3 \mathrm{p}^{2} 3 \mathrm{~d}, 3 \mathrm{~s}^{2} 3 \mathrm{p}^{2} 4 \mathrm{~s}$ configurations and for 59 odd states of the $3 s^{2} 3 p^{3}, 3 s^{2} 3 p^{2} 4 p, 3 s 3 p^{3} 3 d, 3 p^{5}$ configurations in Ar IV. The averaged uncertainty of energy levels obtained in this work compared with the NIST data is $0.21 \%$. The largest disagreement (about 1\%) is just for the first few excited levels.

Raineri et al. (2018) presented ten new energy levels of the $3 s^{2} 3 p^{2} 3 d$ and $3 s^{2} 3 p^{2} 4 p$ configurations for Ar IV. In Table $4 a$ comparison of these levels with this work and theoretical results by Fischer et al. (2006) is made. There is very good agreement between the new energy levels and the present calculations, except for the $3 \mathrm{~s}^{2} 3 \mathrm{p}^{2}\left({ }_{0}^{1} \mathrm{~S}\right){ }^{1} \mathrm{~S} 3 \mathrm{~d}{ }^{2} \mathrm{D}_{5 / 2,7 / 2}$ states for which the relative difference is about $14 \%$. Such a large difference suggests that there is a misidentification and that further experimental analysis is needed.

Transition data for E1, M1, and E2 transitions are given in Table 7, and are available at the CDS. In Fig. 4 a scatterplot of $\mathrm{d} T$ versus the transition rate $A$ is displayed for all presented E1 transitions. The mean $\mathrm{d} T$ for the transitions is $6.47 \%$. Again, for most of the strongest transitions, $\mathrm{d} T$ is well below $2 \%$.

\section{Conclusions}

Energy spectra and transition data of E1, M1, and E2 transitions are presented for S II, Cl III, and Ar IV using MCDHF and RCI methods. The accuracy of the results is evaluated by comparing energy levels with data from NIST database and by the agreement of transition rates between length and velocity gauges. For the first time levels of the $3 \mathrm{~s} 3 \mathrm{p}^{3} 3 \mathrm{~d}$ configuration are presented for the studied elements. The averaged uncertainty of computed energy levels compared with NIST data is $0.22 \%, 0.18 \%$, and $0.21 \%$, respectively for S II, Cl III, and Ar IV ions. The mean $\mathrm{d} T$ for all presented E1 transitions is $12.00 \%, 5.95 \%$, and $6.47 \%$, respectively, for the $\mathrm{S}$ II, $\mathrm{Cl}$ III, and Ar IV ions. 
Table 4. Comparison of new levels by Raineri et al. (2018) with theoretical computations for Ar IV.

\begin{tabular}{|c|c|c|c|c|c|c|}
\hline \multirow[b]{2}{*}{ Label } & \multicolumn{2}{|c|}{ Raineri et al. (2018) } & \multicolumn{2}{|c|}{ This work } & \multicolumn{2}{|c|}{ Fischer et al. (2006) } \\
\hline & Exp. & Cal. & $\mathrm{RCI}(\mathrm{CV})$ & Diff. \% & Calc. & Diff. \% \\
\hline $3 \mathrm{~s}^{2} 3 \mathrm{p}^{2}\left({ }_{2}^{1} \mathrm{D}\right){ }^{1} \mathrm{D} 3 \mathrm{~d}^{2} \mathrm{~F}_{5 / 2}$ & 185795.6 & 185870 & 187234 & 0.77 & 188521.63 & 1.47 \\
\hline $3 \mathrm{~s}^{2} 3 \mathrm{p}^{2}\left({ }_{2}^{1} \mathrm{D}\right){ }^{1} \mathrm{D} 3 \mathrm{~d}^{2} \mathrm{~F}_{7 / 2}$ & 186451.8 & 186591 & 189057 & 1.40 & 189467.32 & 1.62 \\
\hline $3 \mathrm{~s}^{2} 3 \mathrm{p}^{2}\left({ }_{2}^{1} \mathrm{D}\right){ }^{1} \mathrm{D} 3 \mathrm{~d}^{2} \mathrm{P}_{1 / 2}$ & 245175.2 & 245287 & 246701 & 0.62 & 248938.85 & 1.54 \\
\hline $3 \mathrm{~s}^{2} 3 \mathrm{p}^{2}\left({ }_{0}^{1} \mathrm{~S}\right){ }^{1} \mathrm{~S} 3 \mathrm{~d}^{2} \mathrm{D}_{5 / 2}$ & 261761.1 & 261946 & 226733 & -13.38 & 227731.72 & -13.00 \\
\hline $3 \mathrm{~s}^{2} 3 \mathrm{p}^{2}\left({ }_{0}^{1} \mathrm{~S}\right){ }^{1} \mathrm{~S} 3 \mathrm{~d}^{2} \mathrm{D}_{3 / 2}$ & 262626.1 & 262735 & 225349 & -14.19 & 226550.48 & -13.74 \\
\hline $3 \mathrm{~s}^{2} 3 \mathrm{p}^{2}\left({ }_{2}^{3} \mathrm{P}\right){ }^{3} \mathrm{P} 4 \mathrm{p}^{2} \mathrm{~S}_{1 / 2}^{\circ}$ & 282726.0 & 282596 & 280888 & -0.65 & 280500.33 & -0.79 \\
\hline $3 \mathrm{~s}^{2} 3 \mathrm{p}^{2}\left({ }_{2}^{1} \mathrm{D}\right){ }^{1} \mathrm{D} 4 \mathrm{p}^{2} \mathrm{P}_{1 / 2}^{\circ}$ & 311018.3 & 310973 & 312019 & 0.32 & & \\
\hline $3 \mathrm{~s}^{2} 3 \mathrm{p}^{2}\left({ }_{2}^{1} \mathrm{D}\right){ }^{1} \mathrm{D} 4 \mathrm{p}^{2} \mathrm{P}_{3 / 2}^{\circ}$ & 311276.3 & 311201 & 312497 & 0.39 & & \\
\hline $3 \mathrm{~s}^{2} 3 \mathrm{p}^{2}\left({ }_{0}^{1} \mathrm{~S}\right){ }^{1} \mathrm{~S} 4 \mathrm{p}^{2} \mathrm{P}_{1 / 2}^{\circ}$ & 327113.4 & 327126 & 327235 & 0.04 & & \\
\hline $3 \mathrm{~s}^{2} 3 \mathrm{p}^{2}\left({ }_{0}^{1} \mathrm{~S}\right){ }^{1} \mathrm{~S} 4 \mathrm{p}^{2} \mathrm{P}_{3 / 2}^{\circ}$ & 327388.9 & 327333 & 327351 & -0.01 & & \\
\hline
\end{tabular}

Notes. The Diff. columns give the relative difference of the theoretical results compared with the experimental ones.

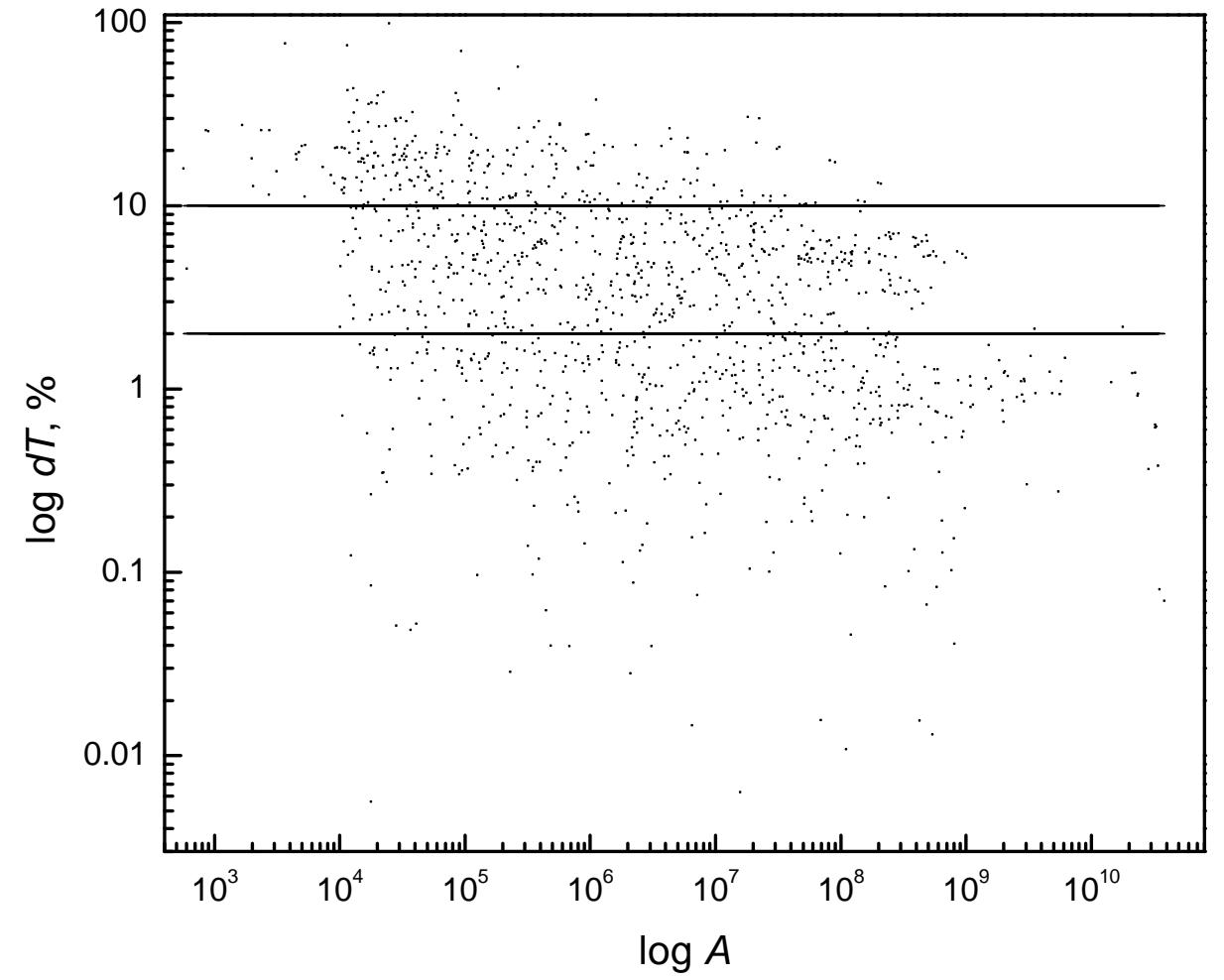

Fig. 4. Scatterplot of $\mathrm{d} T$ vs. the transition rate $A$ of E1 transitions for Ar IV. The solid lines indicate the $2 \%$ and $10 \%$ deviations.
Acknowledgements. This research is funded by the European Social Fund under the No 09.3.3-LMT-K-712 "Development of Competences of Scientists, other Researchers and Students through Practical Research Activities" measure.

\section{References}

Bredice, F., Gallardo, M., Almandos, J. G. R., Trigueiros, A. G., \& Pagan, C. J. B. 1995, Phys. Scr., 51, 446

Burger, M., Skočić, M., Gavrilov, M., Bukvić, S., \& Djeniže, S. 2012, J. Quant. Spectr. Rad. Transf., 113, 1662

Cowan, R. D. 1981, The Theory of Atomic Structure and Spectra (Berkeley, CA, USA: University of California Press)

Djenize, S., \& Bukvić, S. 2001, A\&A, 365, 252

Dyall, K., Grant, I., Johnson, C., Parpia, F., \& Plummer, E. 1989, Comput. Phys. Commun., 55, 425

Ekman, J., Godefroid, M., \& Hartman, H. 2014, Atoms, 2, 215

Fischer, C. F., Tachiev, G., \& Irimia, A. 2006, At. Data Nucl. Data Tables, 92, 607

Fischer, C. F., Godefroid, M., Brage, T., Jönsson, P., \& Gaigalas, G. 2016, J. Phys. B: At. Mol. Opt. Phys., 49, 182004

Fischer, C. F., Gaigalas, G., Jönsson, P., \& Bieroń, J. 2019, Comput. Phys. Commun., 237, 184
Fritzsche, S., Fricke, B., Geschke, D., Heitmann, A., \& Sienkiewicz, J. E. 1999, ApJ, 518, 994

Gaigalas, G., Žalandauskas, T., \& Rudzikas, Z. 2003, At. Data Nucl. Data Tables, 84,99

Gaigalas, G., Fischer, C., Rynkun, P., \& Jönsson, P. 2017, Atoms, 5, 6

Grant, I. P. 2007, Relativistic Quantum Theory of Atoms and Molecules (New York: Springer)

Jönsson, P., Gaigalas, G., Bieroń, J., Fischer, C. F., \& Grant, I. 2013, Comput. Phys. Commun., 184, 2197

Juan de Dios, L., \& Rodríguez, M. 2017, MNRAS, 469, 1036

Kisielius, R., Kulkarni, V. P., Ferland, G. J., Bogdanovich, P., \& Lykins, M. L. 2014, ApJ, 780, 76

Kramida, A., Ralchenko, Yu., Reader, J., \& NIST ASD Team 2018, NIST Atomic Spectra Database (ver. 5.5.6). Available: https://physics.nist. gov/asd, National Institute of Standards and Technology, Gaithersburg, MD

Li, J., Jönsson, P., Dong, C., \& Gaigalas, G. 2010, J. Phys. B: At. Mol. Opt. Phys., 43, 035005

Raineri, M., Mejia Castellanos, R., Gallardo, M., \& Reyna Almandos, J. 2018, Atoms, 6, 47

Schectman, R. M., Federman, S. R., Brown, M., et al. 2005, ApJ, 621, 1159

Sossah, A. M., \& Tayal, S. S. 2012, ApJS, 202, 12

Tayal, S. S., \& Zatsarinny, O. 2010, ApJS, 188, 32

Ynnerman, A., \& Fischer, C. F. 1995, Phys. Rev. A, 51, 2020 


\section{Appendix A: Wave function composition in LS-coupling, energy levels, and lifetimes for the S II, CI III, and Ar IV ions}

Table A.1. Wave function composition (up to three $L S$ components with a contribution $>0.02$ of the total wave function) in $L S$-coupling and energy levels (in $\mathrm{cm}^{-1}$ ) for S II.

\begin{tabular}{|c|c|c|c|c|c|c|}
\hline No. & State & $L S$-composition & $E_{\mathrm{RCI}(\mathrm{CV})}$ & $E_{\mathrm{NIST}}$ & $\tau_{1}$ & $\tau_{\mathrm{v}}$ \\
\hline 1 & $3 s^{2} 3 p^{3}\left({ }_{3}^{4} S\right){ }^{4} S_{3 / 2}^{\circ}$ & $0.93+0.023 \mathrm{~s}^{2} \mathrm{~S} 3 \mathrm{p}^{3}\left({ }_{3}^{2} \mathrm{D}\right){ }^{3} \mathrm{D} 3 \mathrm{~d}^{4} \mathrm{~S}^{\circ}$ & 0 & 0 & & \\
\hline 2 & $3 \mathrm{~s}^{2} 3 \mathrm{p}^{3}\left({ }_{3}^{2} \mathrm{D}\right){ }^{2} \mathrm{D}_{3 / 2}^{\circ}$ & 0.92 & 15122 & 14852.94 & $1.06 \mathrm{e}+03$ & $1.06 \mathrm{e}+03$ \\
\hline 3 & $3 s^{2} 3 p^{3}\left({ }_{3}^{2} \mathrm{D}\right){ }^{2} \mathrm{D}_{5 / 2}^{\mathrm{o} / 2}$ & 0.92 & 15152 & 14884.73 & $3.52 \mathrm{e}+03$ & $3.78 \mathrm{e}+03$ \\
\hline 4 & $3 \mathrm{~s}^{2} 3 \mathrm{p}^{3}\left({ }_{1}^{2} \mathrm{P}\right){ }^{2} \mathrm{P}_{1 / 2}^{\mathrm{o} / 2}$ & $0.90+0.023 \mathrm{p}^{5}{ }^{2} \mathrm{P}^{\circ}$ & 24978 & 24524.83 & $3.01 \mathrm{e}+00$ & $3.10 \mathrm{e}+00$ \\
\hline 5 & $3 \mathrm{~s}^{2} 3 \mathrm{p}^{3}\left({ }_{1}^{2} \mathrm{P}\right){ }^{2} \mathrm{P}_{3 / 2}^{\mathrm{o} / 2}$ & $0.90+0.023 \mathrm{p}^{5}{ }^{2} \mathrm{P}^{\circ}$ & 25016 & 24571.54 & $1.83 \mathrm{e}+00$ & $1.86 \mathrm{e}+00$ \\
\hline 6 & $3 \mathrm{~s}^{2} \mathrm{~S} 3 \mathrm{p}^{4}\left({ }_{2}^{3} \mathrm{P}\right){ }^{4} \mathrm{P}_{5 / 2}^{3 / 2}$ & $0.79+0.143 \mathrm{~s}^{2} 3 \mathrm{p}^{2}\left({ }_{2}^{3} \mathrm{P}\right){ }^{3} \mathrm{P} 3 \mathrm{~d}^{4} \mathrm{P}$ & 78971 & 79395.39 & $2.22 \mathrm{e}-08$ & $2.12 \mathrm{e}-08$ \\
\hline 7 & $3 \mathrm{~s}^{2} \mathrm{~S} 3 \mathrm{p}^{4}\left({ }_{2}^{3} \mathrm{P}\right){ }^{4} \mathrm{P}_{3 / 2}$ & $0.79+0.143 \mathrm{~s}^{2} 3 \mathrm{p}^{2}\left({ }_{2}^{3} \mathrm{P}\right){ }^{3} \mathrm{P} 3 \mathrm{~d}^{4} \mathrm{P}$ & 79326 & 79756.83 & $2.21 \mathrm{e}-08$ & $2.11 \mathrm{e}-08$ \\
\hline 8 & $3 \mathrm{~s}^{2} \mathrm{~S} 3 \mathrm{p}^{4}\left({ }_{2}^{3} \mathrm{P}\right){ }^{4} \mathrm{P}_{1 / 2}$ & $0.79+0.143 \mathrm{~s}^{2} 3 \mathrm{p}^{2}\left({ }_{2}^{3} \mathrm{P}\right){ }^{3} \mathrm{P} 3 \mathrm{~d}^{4} \mathrm{P}$ & 79539 & 79962.61 & $2.20 \mathrm{e}-08$ & $2.11 \mathrm{e}-08$ \\
\hline 9 & $3 \mathrm{~s}^{2} \mathrm{~S} 3 \mathrm{p}^{4}\left({ }_{2}^{1} \mathrm{D}\right){ }^{2} \mathrm{D}_{3 / 2}$ & $0.60+0.253 \mathrm{~s}^{2} 3 \mathrm{p}^{2}\left({ }_{2}^{1} \mathrm{D}\right){ }^{1} \mathrm{D} 3 \mathrm{~d}^{2} \mathrm{D}+0.033 \mathrm{~s}^{2} 3 \mathrm{p}^{2}\left({ }_{0}^{1} \mathrm{~S}\right){ }^{1} \mathrm{~S} 3 \mathrm{~d}^{2} \mathrm{D}$ & 97765 & 97890.74 & $1.84 \mathrm{e}-08$ & $1.70 \mathrm{e}-08$ \\
\hline 10 & $3 \mathrm{~s}^{2} \mathrm{~S} 3 \mathrm{p}^{4}\left({ }_{2}^{1} \mathrm{D}\right){ }^{2} \mathrm{D}_{5 / 2}$ & $0.60+0.253 \mathrm{~s}^{2} 3 \mathrm{p}^{2}\left({ }_{2}^{1} \mathrm{D}\right){ }^{1} \mathrm{D} 3 \mathrm{~d}^{2} \mathrm{D}+0.033 \mathrm{~s}^{2} 3 \mathrm{p}^{2}\left({ }_{0}^{1} \mathrm{~S}\right){ }^{1} \mathrm{~S} 3 \mathrm{~d}^{2} \mathrm{D}$ & 97796 & 97918.86 & $1.91 \mathrm{e}-08$ & $1.75 \mathrm{e}-08$ \\
\hline 11 & $3 \mathrm{~s}^{2} 3 \mathrm{p}^{2}\left({ }_{2}^{3} \mathrm{P}\right){ }^{3} \mathrm{P} 3 \mathrm{~d}^{2} \mathrm{P}_{3 / 2}$ & $0.66+0.163 \mathrm{~s}^{2} \mathrm{~S} 3 \mathrm{p}^{4}\left({ }_{2}^{3} \mathrm{P}\right){ }^{2} \mathrm{P}+0.083 \mathrm{~s}^{2} 3 \mathrm{p}^{2}\left({ }_{2}^{1} \mathrm{D}\right){ }^{1} \mathrm{D} 3 \mathrm{~d}^{2} \mathrm{P}$ & 105506 & 105599.06 & $2.05 \mathrm{e}-08$ & $2.04 \mathrm{e}-08$ \\
\hline 12 & $3 \mathrm{~s}^{2} 3 \mathrm{p}^{2}\left({ }_{2}^{3} \mathrm{P}\right){ }^{3} \mathrm{P} 3 \mathrm{~d}^{2} \mathrm{P}_{1 / 2}$ & $0.66+0.173 \mathrm{~s}^{2} \mathrm{~S} 3 \mathrm{p}^{4}\left({ }_{2}^{3} \mathrm{P}\right){ }^{2} \mathrm{P}+0.073 \mathrm{~s}^{2} 3 \mathrm{p}^{2}\left({ }_{2}^{\mathrm{T}} \mathrm{D}\right){ }^{1} \mathrm{D} 3 \mathrm{~d}^{2} \mathrm{P}$ & 105961 & 106044.24 & $1.98 \mathrm{e}-08$ & $1.97 \mathrm{e}-08$ \\
\hline 13 & $3 \mathrm{~s}^{2} 3 \mathrm{p}^{2}\left({ }_{2}^{3} \mathrm{P}\right){ }^{3} \mathrm{P} 4 \mathrm{~s}^{4} \mathrm{P}_{1 / 2}$ & $0.92 \quad-2$ & 109364 & 109560.69 & $9.64 \mathrm{e}-10$ & $9.62 \mathrm{e}-10$ \\
\hline 14 & $3 \mathrm{~s}^{2} 3 \mathrm{p}^{2}\left({ }_{2}^{2} \mathrm{P}\right){ }^{3} \mathrm{P} 4 \mathrm{~s}^{4} \mathrm{P}_{3 / 2}$ & 0.92 & 109627 & 109831.59 & $9.53 \mathrm{e}-10$ & $9.50 \mathrm{e}-10$ \\
\hline 15 & $3 \mathrm{~s}^{2} 3 \mathrm{p}^{2}\left({ }_{2}^{3} \mathrm{P}\right){ }^{3} \mathrm{P} 3 \mathrm{~d}^{4} \mathrm{~F}_{3 / 2}$ & 0.91 & 110025 & 110177.02 & $3.73 \mathrm{e}-06$ & $3.62 \mathrm{e}-06$ \\
\hline 16 & $3 \mathrm{~s}^{2} 3 \mathrm{p}^{2}\left({ }_{2}^{3} \mathrm{P}\right){ }^{3} \mathrm{P} 4 \mathrm{~s}^{4} \mathrm{P}_{5 / 2}$ & 0.92 & 110059 & 110268.6 & $9.38 \mathrm{e}-10$ & $9.35 \mathrm{e}-10$ \\
\hline 17 & $3 \mathrm{~s}^{2} 3 \mathrm{p}^{2}\left({ }_{2}^{3} \mathrm{P}\right){ }^{3} \mathrm{P} 3 \mathrm{~d}^{4} \mathrm{~F}_{5 / 2}$ & 0.91 & 110161 & 110313.4 & $2.52 \mathrm{e}-06$ & $2.55 \mathrm{e}-06$ \\
\hline 18 & $3 \mathrm{~s}^{2} 3 \mathrm{p}^{2}\left({ }_{2}^{2} \mathrm{P}\right){ }^{3} \mathrm{P} 3 \mathrm{~d}^{4} \mathrm{~F}_{7 / 2}$ & 0.91 & 110362 & 110508.71 & $3.47 \mathrm{e}-06$ & $3.62 \mathrm{e}-06$ \\
\hline 19 & $3 \mathrm{~s}^{2} 3 \mathrm{p}^{2}\left({ }_{2}^{3} \mathrm{P}\right){ }^{3} \mathrm{P} 3 \mathrm{~d}^{4} \mathrm{~F}_{9 / 2}$ & 0.91 & 110636 & 110766.56 & $1.41 \mathrm{e}+00$ & $1.34 \mathrm{e}+00$ \\
\hline 20 & $3 \mathrm{~s}^{2} 3 \mathrm{p}^{2}\left({ }_{2}^{3} \mathrm{P}\right){ }^{3} \mathrm{P} 4 \mathrm{~s}^{2} \mathrm{P}_{1 / 2}$ & 0.91 & 112815 & 112937.57 & $4.75 \mathrm{e}-10$ & $4.72 \mathrm{e}-10$ \\
\hline 21 & $3 \mathrm{~s}^{2} 3 \mathrm{p}^{2}\left({ }_{2}^{3} \mathrm{P}\right){ }^{3} \mathrm{P} 4 \mathrm{~s}^{2} \mathrm{P}_{3 / 2}$ & 0.90 & 113327 & 113461.54 & $4.75 \mathrm{e}-10$ & $4.72 \mathrm{e}-10$ \\
\hline 22 & $3 \mathrm{~s}^{2} 3 \mathrm{p}^{2}\left({ }_{2}^{3} \mathrm{P}\right){ }^{3} \mathrm{P} 3 \mathrm{~d}^{4} \mathrm{D}_{1 / 2}$ & 0.91 & 114002 & 114162.3 & $1.48 \mathrm{e}-06$ & $1.49 \mathrm{e}-06$ \\
\hline 23 & $3 \mathrm{~s}^{2} 3 \mathrm{p}^{2}\left({ }_{2}^{3} \mathrm{P}\right){ }^{3} \mathrm{P} 3 \mathrm{~d}^{4} \mathrm{D}_{3 / 2}$ & 0.91 & 114034 & 114200.54 & $7.91 \mathrm{e}-07$ & $7.98 \mathrm{e}-07$ \\
\hline 24 & $3 s^{2} 3 p^{2}\left({ }_{2}^{3} \mathrm{P}\right){ }^{3} \mathrm{P} 3 \mathrm{~d}^{4} \mathrm{D}_{5 / 2}$ & $0.87+0.023 \mathrm{~s}^{2} 3 \mathrm{p}^{2}\left({ }_{2}^{1} \mathrm{D}\right){ }^{1} \mathrm{D} 3 \mathrm{~d}^{2} \mathrm{~F}+0.023 \mathrm{~s}^{2} 3 \mathrm{p}^{2}\left({ }_{2}^{3} \mathrm{P}\right){ }^{3} \mathrm{P} 3 \mathrm{~d}^{2} \mathrm{~F}$ & 114069 & 114231.04 & $3.30 \mathrm{e}-07$ & $3.37 \mathrm{e}-07$ \\
\hline 25 & $3 s^{2} 3 p^{2}\left({ }_{2}^{3} \mathrm{P}\right){ }^{3} \mathrm{P} 3 \mathrm{~d}^{4} \mathrm{D}_{7 / 2}$ & $0.84+0.043 \mathrm{~s}^{2} 3 \mathrm{p}^{2}\left({ }_{2}^{1} \mathrm{D}\right){ }^{1} \mathrm{D} 3 \mathrm{~d}^{2} \mathrm{~F}+0.033 \mathrm{~s}^{2} 3 \mathrm{p}^{2}\left({ }_{2}^{3} \mathrm{P}\right){ }^{3} \mathrm{P} 3 \mathrm{~d}^{2} \mathrm{~F}$ & 114126 & 114279.33 & $5.86 \mathrm{e}-07$ & $5.97 \mathrm{e}-07$ \\
\hline 26 & $3 \mathrm{~s}^{2} 3 \mathrm{p}^{2}\left({ }_{2}^{1} \mathrm{D}\right){ }^{1} \mathrm{D} 3 \mathrm{~d}^{2} \mathrm{~F}_{5 / 2}$ & $0.44+0.423 \mathrm{~s}^{2} 3 \mathrm{p}^{2}\left({ }_{2}^{3} \mathrm{P}\right){ }^{3} \mathrm{P} 3 \mathrm{~d}^{2} \mathrm{~F}+0.053 \mathrm{~s}^{2} 3 \mathrm{p}^{2}\left({ }_{2}^{3} \mathrm{P}\right){ }^{3} \mathrm{P} 3 \mathrm{~d}^{4} \mathrm{D}$ & 114721 & 114804.37 & $2.42 \mathrm{e}-08$ & $2.48 \mathrm{e}-08$ \\
\hline 27 & $3 \mathrm{~s}^{2} 3 \mathrm{p}^{2}\left({ }_{2}^{1} \mathrm{D}\right){ }^{1} \mathrm{D} 3 \mathrm{~d}^{2} \mathrm{~F}_{7 / 2}$ & $0.43+0.403 \mathrm{~s}^{2} 3 \mathrm{p}^{2}\left({ }_{2}^{3} \mathrm{P}\right){ }^{3} \mathrm{P} 3 \mathrm{~d}^{2} \mathrm{~F}+0.073 \mathrm{~s}^{2} 3 \mathrm{p}^{2}\left({ }_{2}^{3} \mathrm{P}\right){ }^{3} \mathrm{P} 3 \mathrm{~d}^{4} \mathrm{D}$ & 115202 & 115285.61 & $2.97 \mathrm{e}-08$ & $3.03 \mathrm{e}-08$ \\
\hline 28 & $3 \mathrm{~s}^{2} \mathrm{~S} 3 \mathrm{p}^{4}\left({ }_{0}^{1} \mathrm{~S}\right){ }^{2} \mathrm{~S}_{1 / 2}$ & $0.54+0.383 \mathrm{~s}^{2} 3 \mathrm{p}^{2}\left({ }_{2}^{1} \mathrm{D}\right){ }^{1} \mathrm{D} 3 \mathrm{~d}^{2} \mathrm{~S}$ & 119994 & 119783.77 & $6.89 \mathrm{e}-09$ & $6.32 \mathrm{e}-09$ \\
\hline 29 & $3 s^{2} 3 p^{2}\left({ }_{2}^{1} \mathrm{D}\right){ }^{1} \mathrm{D} 4 \mathrm{~s}^{2} \mathrm{D}_{3 / 2}$ & $0.90^{2}$ & 121508 & 121528.72 & $7.15 \mathrm{e}-10$ & $7.13 \mathrm{e}-10$ \\
\hline 30 & $3 \mathrm{~s}^{2} 3 \mathrm{p}^{2}\left({ }_{2}^{1} \mathrm{D}\right){ }^{1} \mathrm{D} 4 \mathrm{~s}^{2} \mathrm{D}_{5 / 2}$ & 0.90 & 121511 & 121530.02 & $7.04 \mathrm{e}-10$ & $7.02 \mathrm{e}-10$ \\
\hline 31 & $3 \mathrm{~s}^{2} 3 \mathrm{p}^{2}\left({ }_{2}^{3} \mathrm{P}\right){ }^{3} \mathrm{P} 4 \mathrm{p}^{2} \mathrm{~S}_{1 / 2}^{\circ}$ & 0.92 & 125286 & 125485.29 & $1.54 \mathrm{e}-08$ & $1.57 \mathrm{e}-08$ \\
\hline 32 & $3 s^{2} 3 p^{2}\left({ }_{2}^{1} D\right){ }^{1} D 3 d^{2} G_{7 / 2}$ & 0.92 & 127207 & 127127.1 & $4.73 \mathrm{e}-07$ & $4.79 \mathrm{e}-07$ \\
\hline 33 & $3 s^{2} 3 p^{2}\left({ }_{2}^{1} D\right){ }^{1} D 3 d^{2} G_{9 / 2}$ & 0.92 & 127243 & 127128.35 & $4.78 \mathrm{e}-01$ & $4.87 \mathrm{e}-01$ \\
\hline 34 & $3 \mathrm{~s}^{2} 3 \mathrm{p}^{2}\left({ }_{2}^{3} \mathrm{P}\right){ }^{3} \mathrm{P} 4 \mathrm{p}^{4} \mathrm{D}_{1 / 2}^{\circ}$ & 0.92 & 127613 & 127825.08 & $8.82 \mathrm{e}-09$ & $8.99 \mathrm{e}-09$ \\
\hline 35 & $3 \mathrm{~s}^{2} 3 \mathrm{p}^{2}\left({ }_{2}^{3} \mathrm{P}\right){ }^{3} \mathrm{P} 4 \mathrm{p}^{4} \mathrm{D}_{3 / 2}^{\circ}$ & 0.92 & 127757 & 127976.34 & $8.78 \mathrm{e}-09$ & $8.94 \mathrm{e}-09$ \\
\hline 36 & $3 s^{2} 3 p^{2}\left({ }_{2}^{3} \mathrm{P}\right){ }^{3} \mathrm{P} 4 \mathrm{p}^{4} \mathrm{D}_{5 / 2}^{\mathrm{o} / 2}$ & 0.92 & 128009 & 128233.2 & $8.73 \mathrm{e}-09$ & $8.89 \mathrm{e}-09$ \\
\hline 37 & $3 s^{2} 3 p^{2}\left({ }_{2}^{3} \mathrm{P}\right){ }^{3} \mathrm{P} 4 \mathrm{p}^{4} \mathrm{D}_{7 / 2}^{\circ}$ & 0.92 & 128374 & 128599.16 & $8.69 \mathrm{e}-09$ & $8.85 \mathrm{e}-09$ \\
\hline 38 & $3 \mathrm{~s}^{2} 3 \mathrm{p}^{2}\left({ }_{2}^{3} \mathrm{P}\right){ }^{3} \mathrm{P} 4 \mathrm{p}^{4} \mathrm{P}_{1 / 2}^{\circ}$ & 0.92 & 129555 & 129787.83 & $7.14 \mathrm{e}-09$ & $7.25 \mathrm{e}-09$ \\
\hline 39 & $3 \mathrm{~s}^{2} 3 \mathrm{p}^{2}\left({ }_{2}^{3} \mathrm{P}\right){ }^{3} \mathrm{P} 4 \mathrm{p}^{4} \mathrm{P}_{3 / 2}^{\mathrm{o}}$ & $0.86+0.043 \mathrm{~s}^{2} 3 \mathrm{p}^{2}\left({ }_{2}^{3} \mathrm{P}\right){ }^{3} \mathrm{P} 4 \mathrm{p}^{4} \mathrm{~S}^{\circ}$ & 129617 & 129858.18 & $7.01 \mathrm{e}-09$ & $7.11 \mathrm{e}-09$ \\
\hline 40 & $3 \mathrm{~s}^{2} 3 \mathrm{p}^{2}\left({ }_{2}^{3} \mathrm{P}\right){ }^{3} \mathrm{P} 4 \mathrm{p}^{4} \mathrm{P}_{5 / 2}^{\circ}$ & $0.88+0.033 \mathrm{~s}^{2} 3 \mathrm{p}^{2}\left({ }_{2}^{3} \mathrm{P}\right){ }^{3} \mathrm{P} 4 \mathrm{p}^{2} \mathrm{D}^{\circ}$ & 129892 & 130134.16 & $7.23 \mathrm{e}-09$ & $7.33 \mathrm{e}-09$ \\
\hline 41 & $3 \mathrm{~s}^{2} 3 \mathrm{p}^{2}\left({ }_{2}^{3} \mathrm{P}\right){ }^{3} \mathrm{P} 4 \mathrm{p}^{2} \mathrm{D}_{3 / 2}^{\circ}$ & $0.81+0.093 \mathrm{~s}^{2} 3 \mathrm{p}^{2}\left({ }_{2}^{1} \mathrm{D}\right){ }^{1} \mathrm{D} 4 \mathrm{p}^{2} \mathrm{D}^{\circ}$ & 130468 & 130641.11 & $1.13 \mathrm{e}-08$ & $1.13 \mathrm{e}-08$ \\
\hline 42 & $3 \mathrm{~s}^{2} 3 \mathrm{p}^{2}\left({ }_{2}^{3} \mathrm{P}\right){ }^{3} \mathrm{P} 3 \mathrm{~d}^{4} \mathrm{P}_{5 / 2}^{3 / 2}$ & $0.76+0.103 \mathrm{~s}^{2} \mathrm{~S} 3 \mathrm{p}^{4}\left({ }_{2}^{3} \mathrm{P}\right){ }^{4} \mathrm{P}+0.033 \mathrm{~s}^{2} 3 \mathrm{p}^{2}\left({ }_{2}^{3} \mathrm{P}\right){ }^{3} \mathrm{P} 4 \mathrm{~d}^{4} \mathrm{P}$ & 130728 & 130602.21 & $1.10 \mathrm{e}-10$ & $1.11 \mathrm{e}-10$ \\
\hline 43 & $3 \mathrm{~s}^{2} 3 \mathrm{p}^{2}\left({ }_{2}^{3} \mathrm{P}\right){ }^{3} \mathrm{P} 4 \mathrm{p}^{4} \mathrm{~S}_{3 / 2}^{\circ}$ & $0.86+0.053 \mathrm{~s}^{2} 3 \mathrm{p}^{2}\left({ }_{2}^{3} \mathrm{P}\right){ }^{3} \mathrm{P} 4 \mathrm{p}^{4} \mathrm{P}^{\circ}$ & 130749 & 131028.85 & $5.04 \mathrm{e}-09$ & $5.16 \mathrm{e}-09$ \\
\hline 44 & $3 \mathrm{~s}^{2} 3 \mathrm{p}^{2}\left({ }_{2}^{3} \mathrm{P}\right){ }^{3} \mathrm{P} 3 \mathrm{~d}^{4} \mathrm{P}_{3 / 2}$ & $0.76+0.113 \mathrm{~s}^{2} \mathrm{~S} 3 \mathrm{p}^{4}\left({ }_{2}^{3} \mathrm{P}\right){ }^{4} \mathrm{P}+0.033 \mathrm{~s}^{2} 3 \mathrm{p}^{2}\left({ }_{2}^{3} \mathrm{P}\right){ }^{3} \mathrm{P} 4 \mathrm{~d}^{4} \mathrm{P}$ & 130942 & 130818.85 & $1.10 \mathrm{e}-10$ & $1.10 \mathrm{e}-10$ \\
\hline 45 & $3 \mathrm{~s}^{2} 3 \mathrm{p}^{2}\left({ }_{2}^{3} \mathrm{P}\right){ }^{3} \mathrm{P} 4 \mathrm{p}^{2} \mathrm{D}_{5 / 2}^{\circ}$ & $0.79+0.083 \mathrm{~s}^{2} 3 \mathrm{p}^{2}\left({ }_{2}^{1} \mathrm{D}\right){ }^{1} \mathrm{D} 4 \mathrm{p}^{2} \mathrm{D}^{\circ}+0.043 \mathrm{~s}^{2} 3 \mathrm{p}^{2}\left({ }_{2}^{3} \mathrm{P}\right){ }^{3} \mathrm{P} 4 \mathrm{p}^{4} \mathrm{P}^{\circ}$ & 131005 & 131187.19 & $1.11 \mathrm{e}-08$ & $1.11 \mathrm{e}-08$ \\
\hline 46 & $3 \mathrm{~s}^{2} 3 \mathrm{p}^{2}\left({ }_{2}^{3} \mathrm{P}\right){ }^{3} \mathrm{P} 3 \mathrm{~d}^{4} \mathrm{P}_{1 / 2}^{5 / 2}$ & $0.76+0.113 \mathrm{~s}^{2} \mathrm{~S} 3 \mathrm{p}^{4}\left({ }_{2}^{3} \mathrm{P}\right){ }^{4} \mathrm{P}+0.033 \mathrm{~s}^{2} 3 \mathrm{p}^{2}\left({ }_{2}^{3} \mathrm{P}\right){ }^{3} \mathrm{P} 4 \mathrm{~d}^{4} \mathrm{P}$ & 131092 & 130948.94 & $1.09 \mathrm{e}-10$ & $1.10 \mathrm{e}-10$ \\
\hline 47 & $3 \mathrm{~s}^{2} 3 \mathrm{p}^{2}\left({ }_{2}^{3} \mathrm{P}\right){ }^{3} \mathrm{P} 4 \mathrm{p}^{2} \mathrm{P}_{1 / 2}^{\circ}$ & $0.83+0.063 \mathrm{~s}^{2} 3 \mathrm{p}^{2}\left({ }_{2}^{1} \mathrm{D}\right){ }^{1} \mathrm{D} 4 \mathrm{p}^{2} \mathrm{P}^{\mathrm{o}}$ & 133125 & 133268.68 & $8.01 \mathrm{e}-09$ & $8.04 \mathrm{e}-09$ \\
\hline 48 & $3 \mathrm{~s}^{2} 3 \mathrm{p}^{2}\left({ }_{2}^{3} \mathrm{P}\right){ }^{3} \mathrm{P} 4 \mathrm{p}^{2} \mathrm{P}_{3 / 2}^{\mathrm{o}}$ & $0.82+0.083 \mathrm{~s}^{2} 3 \mathrm{p}^{2}\left({ }_{2}^{1} \mathrm{D}\right){ }^{1} \mathrm{D} 4 \mathrm{p}^{2} \mathrm{P}^{\circ}$ & 133245 & 133399.97 & $8.00 \mathrm{e}-09$ & $8.02 \mathrm{e}-09$ \\
\hline 49 & $3 \mathrm{~s}^{2} 3 \mathrm{p}^{2}\left({ }_{2}^{3} \mathrm{P}\right){ }^{3} \mathrm{P} 3 \mathrm{~d}^{2} \mathrm{D}_{3 / 2}$ & $0.72+0.113 \mathrm{~s}^{2} 3 \mathrm{p}^{2}\left({ }_{0}^{1} \mathrm{~S}\right){ }^{1} \mathrm{~S} 3 \mathrm{~d}^{2} \mathrm{D}+0.043 \mathrm{~s}^{2} 3 \mathrm{p}^{2}\left({ }_{2}^{3} \mathrm{P}\right){ }^{3} \mathrm{P} 4 \mathrm{~d}^{2} \mathrm{D}$ & 133387 & 133360.86 & $4.00 \mathrm{e}-10$ & $4.05 \mathrm{e}-10$ \\
\hline 50 & $3 \mathrm{~s}^{2} 3 \mathrm{p}^{2}\left({ }_{2}^{3} \mathrm{P}\right){ }^{3} \mathrm{P} 3 \mathrm{~d}^{2} \mathrm{D}_{5 / 2}$ & $0.74+0.103 \mathrm{~s}^{2} 3 \mathrm{p}^{2}\left({ }_{0}^{1} \mathrm{~S}\right){ }^{1} \mathrm{~S} 3 \mathrm{~d}^{2} \mathrm{D}+0.043 \mathrm{~s}^{2} 3 \mathrm{p}^{2}\left({ }_{2}^{3} \mathrm{P}\right){ }^{3} \mathrm{P} 4 \mathrm{~d}^{2} \mathrm{D}$ & 133832 & 133814.84 & $3.72 \mathrm{e}-10$ & $3.76 \mathrm{e}-10$ \\
\hline 51 & $3 s^{2} 3 p^{2}\left({ }^{1} S\right){ }^{1} S 4 s^{2} S_{1 / 2}$ & $0.84+0.043 \mathrm{~s}^{2} 3 \mathrm{p}^{2}\left({ }_{2}^{1} \mathrm{D}\right){ }^{1} \mathrm{D} 3 \mathrm{~d}^{2} \mathrm{~S}+0.043 \mathrm{p}^{4}\left({ }_{0}^{1} \mathrm{~S}\right){ }^{1} \mathrm{~S} 4 \mathrm{~s}^{2} \mathrm{~S}$ & 136386 & 136328.79 & $3.33 \mathrm{e}-10$ & $3.33 \mathrm{e}-10$ \\
\hline 52 & $3 \mathrm{~s}^{2} 3 \mathrm{p}^{2}\left({ }_{2}^{3} \mathrm{P}\right){ }^{3} \mathrm{P} 3 \mathrm{~d}^{2} \mathrm{~F}_{5 / 2}$ & $0.41+0.373 \mathrm{~s}^{2} 3 \mathrm{p}^{2}\left({ }_{2}^{1} \mathrm{D}\right){ }^{1} \mathrm{D} 3 \mathrm{~d}^{2} \mathrm{~F}+0.113 \mathrm{~s}^{2} 3 \mathrm{p}^{2}\left({ }_{2}^{3} \mathrm{P}\right){ }^{3} \mathrm{P} 4 \mathrm{~d}^{2} \mathrm{~F}$ & 138586 & 138509.17 & $1.31 \mathrm{e}-10$ & $1.32 \mathrm{e}-10$ \\
\hline 53 & $3 \mathrm{~s}^{2} 3 \mathrm{p}^{2}\left({ }_{2}^{2} \mathrm{P}\right){ }^{3} \mathrm{P} 3 \mathrm{~d}^{2} \mathrm{~F}_{7 / 2}$ & $0.42+0.373 \mathrm{~s}^{2} 3 \mathrm{p}^{2}\left({ }_{2}^{1} \mathrm{D}\right){ }^{1} \mathrm{D} 3 \mathrm{~d}^{2} \mathrm{~F}+0.103 \mathrm{~s}^{2} 3 \mathrm{p}^{2}\left({ }_{2}^{2} \mathrm{P}\right){ }^{3} \mathrm{P} 4 \mathrm{~d}^{2} \mathrm{~F}$ & 138609 & 138527.98 & $1.29 \mathrm{e}-10$ & $1.31 \mathrm{e}-10$ \\
\hline 54 & $3 \mathrm{~s}^{2} 3 \mathrm{p}^{2}\left({ }_{2}^{1} \mathrm{D}\right){ }^{1} \mathrm{D} 3 \mathrm{~d}^{2} \mathrm{P}_{1 / 2}$ & $0.79+0.073 \mathrm{~s}^{2} 3 \mathrm{p}^{2}\left({ }_{2}^{3} \mathrm{P}\right){ }^{3} \mathrm{P} 3 \mathrm{~d}^{2} \mathrm{P}+0.033 \mathrm{~s}^{2} 3 \mathrm{p}^{2}\left({ }_{2}^{3} \mathrm{P}\right){ }^{3} \mathrm{P} 4 \mathrm{~d}^{2} \mathrm{P}$ & 140134 & 139844.99 & $2.24 \mathrm{e}-10$ & $2.31 \mathrm{e}-10$ \\
\hline 55 & $3 \mathrm{~s}^{2} 3 \mathrm{p}^{2}\left({ }_{2}^{1} \mathrm{D}\right){ }^{1} \mathrm{D} 4 \mathrm{p}^{2} \mathrm{~F}_{5 / 2}^{\circ}$ & 0.90 & 140197 & 140230.1 & $9.05 \mathrm{e}-09$ & $9.11 \mathrm{e}-09$ \\
\hline 56 & $3 \mathrm{~s}^{2} 3 \mathrm{p}^{2}\left({ }_{2}^{1} \mathrm{D}\right){ }^{1} \mathrm{D} 4 \mathrm{p}^{2} \mathrm{~F}_{7 / 2}^{\mathrm{o} / 2}$ & 0.92 & 140289 & 140319.23 & $9.01 \mathrm{e}-09$ & $9.05 \mathrm{e}-09$ \\
\hline
\end{tabular}

Notes. Energy levels are given relative to the ground state. The states 65, 102, and 107 are indicated in bold. The labels for these levels were not assigned with largest contribution to the composition. 
Table A.1. continued.

\begin{tabular}{|c|c|c|c|c|c|c|}
\hline 57 & $3 s^{2} 3 p^{2}\left({ }_{2}^{1} \mathrm{D}\right){ }^{1} \mathrm{D} 3 \mathrm{~d}^{2} \mathrm{P}_{3 / 2}$ & $0.79+0.063 \mathrm{~s}^{2} 3 \mathrm{p}^{2}\left({ }_{2}^{3} \mathrm{P}\right){ }^{3} \mathrm{P} 3 \mathrm{~d}^{2} \mathrm{P}+0.023 \mathrm{~s}^{2} 3 \mathrm{p}^{2}\left({ }_{2}^{3} \mathrm{P}\right){ }^{3} \mathrm{P} 4 \mathrm{~d}^{2} \mathrm{P}$ & 140298 & 140016.77 & $2.22 \mathrm{e}-10$ & $2.29 \mathrm{e}-10$ \\
\hline 58 & $3 \mathrm{~s}^{2} 3 \mathrm{p}^{2}\left({ }_{2}^{1} \mathrm{D}\right){ }^{1} \mathrm{D} 4 \mathrm{p}^{2} \mathrm{D}_{5 / 2}^{\circ}$ & $0.80+0.083 \mathrm{~s}^{2} 3 \mathrm{p}^{2}\left({ }_{2}^{3} \mathrm{P}\right){ }^{3} \mathrm{P} 4 \mathrm{p}^{2} \mathrm{D}^{\circ}$ & 140642 & 140708.89 & $6.76 \mathrm{e}-09$ & $6.90 \mathrm{e}-09$ \\
\hline 59 & $3 \mathrm{~s}^{2} 3 \mathrm{p}^{2}\left({ }_{2}^{1} \mathrm{D}\right){ }^{1} \mathrm{D} 4 \mathrm{p}^{2} \mathrm{D}_{3 / 2}^{\circ}$ & $0.81+0.083 \mathrm{~s}^{2} 3 \mathrm{p}^{2}\left({ }_{2}^{3} \mathrm{P}\right){ }^{3} \mathrm{P} 4 \mathrm{p}^{2} \mathrm{D}^{\circ}$ & 140688 & 140750.34 & $6.73 \mathrm{e}-09$ & $6.88 \mathrm{e}-09$ \\
\hline 60 & $3 \mathrm{~s}^{2} 3 \mathrm{p}^{2}\left({ }_{2}^{1} \mathrm{D}\right){ }^{1} \mathrm{D} 4 \mathrm{p}^{2} \mathrm{P}_{1 / 2}^{\circ}$ & $0.83+0.053 \mathrm{~s}^{2} 3 \mathrm{p}^{2}\left({ }_{2}^{3} \mathrm{P}\right){ }^{3} \mathrm{P} 4 \mathrm{p}^{2} \mathrm{P}^{\circ}+0.023 \mathrm{~s}^{2} 3 \mathrm{p}^{2}\left({ }_{2}^{3} \mathrm{P}\right){ }^{3} \mathrm{P} 5 \mathrm{p}^{2} \mathrm{P}^{\circ}$ & 143493 & 143488.95 & $5.79 \mathrm{e}-09$ & $5.86 \mathrm{e}-09$ \\
\hline 61 & $3 \mathrm{~s}^{2} 3 \mathrm{p}^{2}\left({ }_{2}^{1} \mathrm{D}\right){ }^{1} \mathrm{D} 4 \mathrm{p}^{2} \mathrm{P}_{3 / 2}^{\circ}$ & $0.81+0.073 \mathrm{~s}^{2} 3 \mathrm{p}^{2}\left({ }_{2}^{3} \mathrm{P}\right){ }^{3} \mathrm{P} 4 \mathrm{p}^{2} \mathrm{P}^{\circ}+0.023 \mathrm{~s}^{2} 3 \mathrm{p}^{2}\left({ }_{2}^{3} \mathrm{P}\right){ }^{3} \mathrm{P} 5 \mathrm{p}^{2} \mathrm{P}^{\circ}$ & 143613 & 143623.56 & $5.79 \mathrm{e}-09$ & $5.87 \mathrm{e}-09$ \\
\hline 62 & $3 \mathrm{~s}^{2} 3 \mathrm{p}^{2}\left({ }_{2}^{1} \mathrm{D}\right){ }^{1} \mathrm{D} 3 \mathrm{~d}^{2} \mathrm{D}_{5 / 2}$ & $0.51+0.193 \mathrm{~s}^{2} 3 \mathrm{p}^{2}\left({ }_{0}^{1} \mathrm{~S}\right){ }^{1} \mathrm{~S} 3 \mathrm{~d}^{2} \mathrm{D}+0.103 \mathrm{~s}^{2} \mathrm{~S} 3 \mathrm{p}^{4}\left({ }_{2}^{1} \mathrm{D}\right){ }^{2} \mathrm{D}$ & 144308 & 144009.42 & $2.02 \mathrm{e}-10$ & $2.04 \mathrm{e}-10$ \\
\hline 63 & $3 \mathrm{~s}^{2} 3 \mathrm{p}^{2}\left({ }_{2}^{1} \mathrm{D}\right){ }^{1} \mathrm{D} 3 \mathrm{~d}^{2} \mathrm{D}_{3 / 2}$ & $0.52+0.163 \mathrm{~s}^{2} 3 \mathrm{p}^{2}\left({ }_{0}^{1} \mathrm{~S}\right){ }^{1} \mathrm{~S} 3 \mathrm{~d}^{2} \mathrm{D}+0.113 \mathrm{~s}^{2} \mathrm{~S} 3 \mathrm{p}^{4}\left({ }_{2}^{1} \mathrm{D}\right){ }^{2} \mathrm{D}$ & 144459 & 144142.16 & $1.89 \mathrm{e}-10$ & $1.90 \mathrm{e}-10$ \\
\hline 64 & $3 \mathrm{~s}^{2} 3 \mathrm{p}^{2}\left({ }_{2}^{3} \mathrm{P}\right){ }^{3} \mathrm{P} 4 \mathrm{~d}^{2} \mathrm{P}_{3 / 2}$ & $0.36+0.333 \mathrm{~s}^{2} \mathrm{~S} 3 \mathrm{p}^{4}\left({ }_{3}^{3} \mathrm{P}\right){ }^{2} \mathrm{P}+0.133 \mathrm{~s}^{2} 3 \mathrm{p}^{2}\left({ }^{3} \mathrm{P}\right){ }^{3} \mathrm{P} 3 \mathrm{~d}^{2} \mathrm{P}$ & 146144 & 145505.74 & $1.71 \mathrm{e}-10$ & $1.70 \mathrm{e}-10$ \\
\hline 65 & $3 \mathrm{~s}{ }^{2} \mathrm{~S} 3 \mathrm{p}^{4}\left({ }_{2}^{3} \mathrm{P}\right){ }^{2} \mathrm{P}_{1 / 2}$ & $\mathbf{0 . 3 3}+0.353 \mathrm{~s}^{2} 3 \mathrm{p}^{2}\left({ }_{2}^{3} \mathrm{P}\right){ }^{3} \mathrm{P} 4 \mathrm{~d}^{2} \mathrm{P}+0.133 \mathrm{~s}^{2} 3 \mathrm{p}^{2}\left({ }_{2}^{3} \mathrm{P}\right){ }^{3} \mathrm{P} 3 \mathrm{~d}^{2} \mathrm{P}$ & 146549 & 145877.66 & $1.69 \mathrm{e}-10$ & $1.69 \mathrm{e}-10$ \\
\hline 66 & $3 s^{2} 3 p^{2}\left({ }_{0}^{1} S\right){ }^{2} S 3 d^{2} D_{3 / 2}$ & $0.48+0.153 \mathrm{~s}^{2} 3 \mathrm{p}^{2}\left({ }_{2}^{3} \mathrm{P}\right){ }^{3} \mathrm{P} 4 \mathrm{~d}^{2} \mathrm{D}+0.103 \mathrm{~s}^{2} 3 \mathrm{p}^{2}\left({ }_{2}^{2} \mathrm{D}\right){ }^{1} \mathrm{D} 3 \mathrm{~d}^{2} \mathrm{D}$ & 149188 & 148900.91 & $1.68 \mathrm{e}-10$ & $1.69 \mathrm{e}-10$ \\
\hline 67 & $3 \mathrm{~s}^{2} 3 \mathrm{p}^{2}\left({ }_{0}^{1} \mathrm{~S}\right){ }^{1} \mathrm{~S} 3 \mathrm{~d}^{2} \mathrm{D}_{5 / 2}$ & $0.48+0.133 \mathrm{~s}^{2} 3 \mathrm{p}^{2}\left({ }_{2}^{3} \mathrm{P}\right){ }^{3} \mathrm{P} 4 \mathrm{~d}^{2} \mathrm{D}+0.113 \mathrm{~s}^{2} 3 \mathrm{p}^{2}\left({ }_{2}^{1} \mathrm{D}\right){ }^{1} \mathrm{D} 3 \mathrm{~d}^{2} \mathrm{D}$ & 149194 & 148886.57 & $1.61 \mathrm{e}-10$ & $1.62 \mathrm{e}-10$ \\
\hline 68 & $3 s^{2} 3 p^{2}\left({ }_{2}^{3} \mathrm{P}\right){ }^{3} \mathrm{P} 5 \mathrm{~s}^{4} \mathrm{P}_{1 / 2}$ & 0.91 & 149987 & 150258.51 & $2.36 \mathrm{e}-09$ & $2.38 \mathrm{e}-09$ \\
\hline 69 & $3 \mathrm{~s}^{2} 3 \mathrm{p}^{2}\left({ }_{2}^{2} \mathrm{P}\right){ }^{3} \mathrm{P} 5 \mathrm{~s}^{4} \mathrm{P}_{3 / 2}$ & 0.91 & 150251 & 150531.31 & $2.29 \mathrm{e}-09$ & $2.32 \mathrm{e}-09$ \\
\hline 70 & $3 \mathrm{~s}^{2} 3 \mathrm{p}^{2}\left({ }_{2}^{3} \mathrm{P}\right){ }^{3} \mathrm{P} 5 \mathrm{~s}^{4} \mathrm{P}_{5 / 2}$ & 0.92 & 150712 & 150996.41 & $2.24 \mathrm{e}-09$ & $2.26 \mathrm{e}-09$ \\
\hline 71 & $3 \mathrm{~s}^{2} 3 \mathrm{p}^{2}\left({ }_{2}^{3} \mathrm{P}\right){ }^{3} \mathrm{P} 5 \mathrm{~s}^{2} \mathrm{P}_{1 / 2}$ & $0.87+0.033 \mathrm{~s}^{2} 3 \mathrm{p}^{2}\left({ }_{2}^{3} \mathrm{P}\right){ }^{3} \mathrm{P} 4 \mathrm{~d}^{2} \mathrm{P}$ & 151111 & 151383.81 & $1.16 \mathrm{e}-09$ & $1.16 \mathrm{e}-09$ \\
\hline 72 & $3 \mathrm{~s}^{2} 3 \mathrm{p}^{2}\left({ }_{2}^{3} \mathrm{P}\right){ }^{3} \mathrm{P} 5 \mathrm{~s}^{2} \mathrm{P}_{3 / 2}$ & $0.87+0.033 \mathrm{~s}^{2} 3 \mathrm{p}^{2}\left({ }_{2}^{3} \mathrm{P}\right){ }^{3} \mathrm{P} 4 \mathrm{~d}^{2} \mathrm{P}$ & 151623 & 151910.83 & $1.13 \mathrm{e}-09$ & $1.13 \mathrm{e}-09$ \\
\hline 73 & $3 s^{2} 3 p^{2}\left({ }_{2}^{5} \mathrm{P}\right){ }^{3} \mathrm{P} 4 d^{4} \mathrm{~F}_{3 / 2}$ & 0.89 & 151784 & 151959.69 & $4.28 \mathrm{e}-09$ & $4.38 \mathrm{e}-09$ \\
\hline 74 & $3 \mathrm{~s}^{2} 3 \mathrm{p}^{2}\left({ }_{2}^{2} \mathrm{P}\right){ }^{3} \mathrm{P} 4 \mathrm{~d}^{4} \mathrm{~F}_{5 / 2}$ & $87+0.033 \mathrm{~s}^{2} 3 \mathrm{p}^{2}\left({ }_{2}^{3} \mathrm{P}\right){ }^{3} \mathrm{P} 4 \mathrm{~d}^{4} \mathrm{D}$ & 151917 & 152094.64 & $4.27 \mathrm{e}-09$ & $4.37 \mathrm{e}-09$ \\
\hline 75 & $3 \mathrm{~s}^{2} 3 \mathrm{p}^{2}\left({ }_{2}^{3} \mathrm{P}\right){ }^{3} \mathrm{P} 4 \mathrm{~d}^{4} \mathrm{~F}_{7 / 2}$ & $0.87+0.043 \mathrm{~s}^{2} 3 \mathrm{p}^{2}\left({ }_{2}^{3} \mathrm{P}\right){ }^{3} \mathrm{P} 4 \mathrm{~d}^{4} \mathrm{D}$ & 152129 & 152305 & $4.28 \mathrm{e}-09$ & $4.38 \mathrm{e}-09$ \\
\hline 76 & $3 s^{2} 3 p^{2}\left({ }_{2}^{1} D\right){ }^{1} \mathrm{D} 3 d^{2} S_{1 / 2}$ & $0.44+0.253 \mathrm{~s}^{2} \mathrm{~S} 3 \mathrm{p}^{4}\left({ }_{0}^{1} \mathrm{~S}\right){ }^{2} \mathrm{~S}+0.133 \mathrm{~s}^{2} 3 \mathrm{p}^{2}\left({ }_{2}^{1} \mathrm{D}\right){ }^{1} \mathrm{D} 4 \mathrm{~d}^{2} \mathrm{~S}$ & 152302 & 151651.72 & $2.11 \mathrm{e}-10$ & $2.12 \mathrm{e}-10$ \\
\hline 77 & $3 \mathrm{~s}^{2} 3 \mathrm{p}^{2}\left({ }_{2}^{3} \mathrm{P}\right){ }^{3} \mathrm{P} 4 \mathrm{~d}^{4} \mathrm{~F}_{9 / 2}$ & 0.91 & 152446 & 152615.46 & $4.36 \mathrm{e}-09$ & $4.46 \mathrm{e}-09$ \\
\hline 78 & $3 \mathrm{~s}^{2} 3 \mathrm{p}^{2}\left({ }_{2}^{3} \mathrm{P}\right){ }^{3} \mathrm{P} 4 \mathrm{~d}^{4} \mathrm{D}_{1 / 2}$ & 0.91 & & 53.9 & -09 & $4.27 \mathrm{e}-09$ \\
\hline 79 & $3 \mathrm{~s}^{2} 3 \mathrm{p}^{2}\left({ }_{2}^{2} \mathrm{P}\right){ }^{3} \mathrm{P} 4 \mathrm{~d}^{4} \mathrm{D}_{3 / 2}$ & 0.89 & 74 & 95 & -09 & e-09 \\
\hline 80 & $3 \mathrm{~s}^{2} 3 \mathrm{p}^{2}\left({ }_{2}^{3} \mathrm{P}\right){ }^{3} \mathrm{P} 4 \mathrm{~d}^{4} \mathrm{D}_{5 / 2}$ & $0.87+0.033 \mathrm{~s}^{2} 3 \mathrm{p}^{2}\left({ }_{2}^{3} \mathrm{P}\right){ }^{3} \mathrm{P} 4 \mathrm{~d}^{4} \mathrm{~F}$ & 153054 & 153283.07 & $4.01 \mathrm{e}-09$ & $4.06 \mathrm{e}-09$ \\
\hline 81 & $3 \mathrm{~s}^{2} 3 \mathrm{p}^{2}\left({ }_{2}^{3} \mathrm{P}\right){ }^{3} \mathrm{P} 4 \mathrm{~d}^{4} \mathrm{D}_{7 / 2}$ & $0.87+0.043 \mathrm{~s}^{2} 3 \mathrm{p}^{2}\left({ }_{2}^{3} \mathrm{P}\right){ }^{3} \mathrm{P} 4 \mathrm{~d}^{4} \mathrm{~F}$ & 153185 & 153413.74 & $4.53 \mathrm{e}-09$ & $4.59 \mathrm{e}-09$ \\
\hline 82 & $3 \mathrm{~s}^{2} 3 \mathrm{p}^{2}\left({ }_{2}^{3} \mathrm{P}\right){ }^{3} \mathrm{P} 4 \mathrm{~d}^{4} \mathrm{P}_{5 / 2}$ & 0.88 & 155684 & 155818.71 & $3.41 \mathrm{e}-10$ & $3.41 \mathrm{e}-10$ \\
\hline 83 & $3 \mathrm{~s}^{2} 3 \mathrm{p}^{2}\left({ }_{2}^{3} \mathrm{P}\right){ }^{3} \mathrm{P} 4 \mathrm{~d}^{4} \mathrm{P}_{3 / 2}$ & 0.88 & 155893 & 156029.54 & $3.37 \mathrm{e}-10$ & $3.37 \mathrm{e}-10$ \\
\hline 84 & $3 \mathrm{~s}^{2} 3 \mathrm{p}^{2}\left({ }_{2}^{3} \mathrm{P}\right){ }^{3} \mathrm{P} 4 \mathrm{~d}^{2} \mathrm{~F}_{5 / 2}$ & $0.72+0.123 \mathrm{~s}^{2} 3 \mathrm{p}^{2}\left({ }_{2}^{1} \mathrm{D}\right){ }^{1} \mathrm{D} 4 \mathrm{~d}^{2} \mathrm{~F}+0$ & 156019 & 156121.7 & $4.68 \mathrm{e}-10$ & $4.73 e-10$ \\
\hline 85 & $3 \mathrm{~s}^{2} 3 \mathrm{p}^{2}\left({ }_{2}^{3} \mathrm{P}\right){ }^{3} \mathrm{P} 4 \mathrm{~d}^{4} \mathrm{P}_{1 / 2}$ & 0.89 & 156022 & 156148.48 & $3.33 \mathrm{e}-10$ & $3.33 \mathrm{e}-10$ \\
\hline 86 & $3 \mathrm{~s}^{2} 3 \mathrm{p}^{2}\left({ }_{2}^{2} \mathrm{P}\right){ }^{3} \mathrm{P} 5 \mathrm{p}^{2} \mathrm{~S}_{1 / 2}^{\circ}$ & $0.78+0.083 \mathrm{~s}^{2} 3 \mathrm{p}^{2}\left({ }_{0}^{1} \mathrm{~S}\right){ }^{1} \mathrm{~S} 4 \mathrm{p}^{2} \mathrm{P}^{\circ}+0.033 \mathrm{~s}^{2} 3 \mathrm{p}^{2}\left({ }_{2}^{3} \mathrm{P}\right)$ & 156104 & & $1.50 \mathrm{e}-08$ & $1.89 \mathrm{e}-08$ \\
\hline 87 & $3 \mathrm{~s}^{2} 3 \mathrm{p}^{2}\left({ }_{0}^{1} \mathrm{~S}\right){ }^{1} \mathrm{~S} 4 \mathrm{p}^{2} \mathrm{P}_{1 / 2}^{\mathrm{o}}$ & $0.69+0.113 \mathrm{~s}^{2} 3 \mathrm{p}^{2}\left({ }_{2}^{3} \mathrm{P}\right){ }^{3} \mathrm{P} 5 \mathrm{p}^{2} \mathrm{~S}^{\circ}+0.073 \mathrm{~s}^{2} 3 \mathrm{p}^{2}\left({ }_{2}^{3} \mathrm{P}\right){ }^{3} \mathrm{P} 5 \mathrm{p}^{2} \mathrm{P}^{\circ}$ & 156355 & 156167.04 & $8.90 \mathrm{e}-09$ & $9.27 \mathrm{e}-09$ \\
\hline 88 & $3 \mathrm{~s}^{2} 3 \mathrm{p}^{2}\left({ }_{0}^{1} \mathrm{~S}\right){ }^{1} \mathrm{~S} 4 \mathrm{p}^{2} \mathrm{P}_{3 / 2}^{\mathrm{o}}$ & $0.80+0.083 \mathrm{~s}^{2} 3 \mathrm{p}^{2}\left({ }_{2}^{3} \mathrm{P}\right){ }^{3} \mathrm{P} 5 \mathrm{p}^{2} \mathrm{P}^{\circ}+0.043 \mathrm{p}^{4}\left({ }_{0}^{1} \mathrm{~S}\right){ }^{1} \mathrm{~S} 4 \mathrm{p}^{2} \mathrm{P}^{\circ}$ & 156391 & 156276.83 & $8.29 \mathrm{e}-09$ & $8.42 \mathrm{e}-09$ \\
\hline 89 & $3 \mathrm{~s}^{2} 3 \mathrm{p}^{2}\left({ }_{2}^{3} \mathrm{P}\right){ }^{3} \mathrm{P} 4 \mathrm{~d}^{2} \mathrm{~F}_{7 / 2}$ & $0.72+0.113 \mathrm{~s}^{2} 3 \mathrm{p}^{2}\left({ }_{2}^{1} \mathrm{D}\right){ }^{1} \mathrm{D} 4 \mathrm{~d}^{2} \mathrm{~F}+0.063 \mathrm{~s}^{2} 3 \mathrm{p}^{2}\left({ }_{2}^{1} \mathrm{D}\right){ }^{1} \mathrm{D} 3 \mathrm{~d}^{2} \mathrm{~F}$ & 15 & 17 & 10 & -10 \\
\hline 90 & $3 \mathrm{~s}^{2} 3 \mathrm{p}^{2}\left({ }_{2}^{2} \mathrm{P}\right){ }^{3} \mathrm{P} 5 \mathrm{p}^{4} \mathrm{D}_{1 / 2}^{\circ}$ & 0.90 & 156588 & 156829.75 & $2.43 \mathrm{e}-08$ & $3.21 \mathrm{e}-08$ \\
\hline 91 & $3 \mathrm{~s}^{2} 3 \mathrm{p}^{2}\left({ }_{2}^{3} \mathrm{P}\right){ }^{3} \mathrm{P} 5 \mathrm{p}^{4} \mathrm{D}_{3 / 2}^{\circ}$ & $0.86+0.063 \mathrm{~s}^{2} 3 \mathrm{p}^{2}\left({ }_{2}^{3} \mathrm{P}\right){ }^{3} \mathrm{P} 5 \mathrm{p}^{4} \mathrm{P}^{\circ}$ & 156687 & 156939.5 & $2.41 \mathrm{e}-08$ & $3.23 \mathrm{e}-08$ \\
\hline 92 & $3 s^{2} 3 p^{2}\left({ }_{2}^{3} \mathrm{P}\right){ }^{3} \mathrm{P} 5 \mathrm{p}^{4} \mathrm{D}_{5 / 2}^{\circ / 2}$ & $0.86+0.063 \mathrm{~s}^{2} 3 \mathrm{p}^{2}\left({ }_{2}^{3} \mathrm{P}\right){ }^{3} \mathrm{P} 5 \mathrm{p}^{4} \mathrm{P}^{\circ}$ & 156917 & 157173.69 & $2.42 \mathrm{e}-08$ & $3.27 \mathrm{e}-08$ \\
\hline 93 & $3 \mathrm{~s}^{2} 3 \mathrm{p}^{2}\left({ }_{2}^{3} \mathrm{P}\right){ }^{3} \mathrm{P} 5 \mathrm{p}^{4} \mathrm{P}_{3 / 2}^{\circ}$ & $0.47+0.393 \mathrm{~s}^{2} 3 \mathrm{p}^{2}\left({ }_{2}^{3} \mathrm{P}\right){ }^{3} \mathrm{P} 5 \mathrm{p}^{4} \mathrm{~S}^{\circ}+0.053 \mathrm{~s}^{2} 3 \mathrm{p}^{2}\left({ }_{2}^{3} \mathrm{P}\right){ }^{3} \mathrm{P} 5 \mathrm{p}^{4} \mathrm{D}^{\circ}$ & 157274 & 157579.68 & $1.11 \mathrm{e}-08$ & $1.40 \mathrm{e}-08$ \\
\hline 94 & $3 \mathrm{~s}^{2} 3 \mathrm{p}^{2}\left({ }_{2}^{3} \mathrm{P}\right){ }^{3} \mathrm{P} 5 \mathrm{p}^{4} \mathrm{D}_{7 / 2}^{\circ}$ & 0.93 & 157303 & 157558.77 & $2.46 \mathrm{e}-08$ & $3.33 \mathrm{e}-08$ \\
\hline 95 & $3 \mathrm{~s}^{2} 3 \mathrm{p}^{2}\left({ }_{2}^{3} \mathrm{P}\right){ }^{3} \mathrm{P} 5 \mathrm{p}^{4} \mathrm{P}_{1 / 2}^{\circ}$ & $0.89+0.023 \mathrm{~s}^{2} 3 \mathrm{p}^{2}\left({ }_{2}^{3} \mathrm{P}\right){ }^{3} \mathrm{P} 5 \mathrm{p}^{2} \mathrm{~S}^{\circ}$ & 157388 & 157677.32 & $1.85 \mathrm{e}-08$ & $2.46 \mathrm{e}-08$ \\
\hline 96 & $3 \mathrm{~s}^{2} 3 \mathrm{p}^{2}\left({ }_{2}^{3} \mathrm{P}\right){ }^{3} \mathrm{P} 5 \mathrm{p}^{4} \mathrm{P}_{5 / 2}^{\mathrm{o}}$ & $0.84+0.073 \mathrm{~s}^{2} 3 \mathrm{p}^{2}\left({ }_{2}^{3} \mathrm{P}\right){ }^{3} \mathrm{P} 5 \mathrm{p}^{4} \mathrm{D}^{\circ}+0.023 \mathrm{~s}^{2} 3 \mathrm{p}^{2}\left({ }_{2}^{3} \mathrm{P}\right){ }^{3} \mathrm{P} 5 \mathrm{p}^{2} \mathrm{D}^{\circ}$ & 157738 & 158038.6 & $1.89 \mathrm{e}-08$ & $2.52 \mathrm{e}-08$ \\
\hline 97 & $3 \mathrm{~s}^{2} 3 \mathrm{p}^{2}\left({ }_{2}^{3} \mathrm{P}\right){ }^{3} \mathrm{P} 5 \mathrm{p}^{4} \mathrm{~S}_{3 / 2}^{\mathrm{o}}$ & $0.50+0.373 \mathrm{~s}^{2} 3 \mathrm{p}^{2}\left({ }_{2}^{3} \mathrm{P}\right){ }^{3} \mathrm{P} 5 \mathrm{p}^{4} \mathrm{P}^{\circ}+0.033 \mathrm{~s}^{2} 3 \mathrm{p}^{2}\left({ }_{2}^{3} \mathrm{P}\right){ }^{3} \mathrm{P} 5 \mathrm{p}^{2} \mathrm{D}^{\circ}$ & 157801 & 158118.75 & $9.94 \mathrm{e}-09$ & $1.23 \mathrm{e}-08$ \\
\hline 98 & $3 \mathrm{~s}^{2} 3 \mathrm{p}^{2}\left({ }_{2}^{3} \mathrm{P}\right){ }^{3} \mathrm{P} 5 \mathrm{p}^{2} \mathrm{D}_{3 / 2}^{\circ}$ & $0.85+0.043 \mathrm{~s}^{2} 3 \mathrm{p}^{2}\left({ }_{2}^{3} \mathrm{P}\right){ }^{3} \mathrm{P} 5 \mathrm{p}^{4} \mathrm{P}^{\circ}$ & 157947 & 158215.59 & $1.91 \mathrm{e}-08$ & $2.60 \mathrm{e}-08$ \\
\hline 99 & $3 \mathrm{~s}^{2} 3 \mathrm{p}^{2}\left({ }_{2}^{3} \mathrm{P}\right){ }^{3} \mathrm{P} 5 \mathrm{p}^{2} \mathrm{D}_{5 / 2}^{\circ / 2}$ & $0.87+0.033 \mathrm{~s}^{2} 3 \mathrm{p}^{2}\left({ }_{2}^{3} \mathrm{P}\right){ }^{3} \mathrm{P} 5 \mathrm{p}^{4} \mathrm{P}^{\circ}$ & 158442 & 158715.46 & $1.89 \mathrm{e}-08$ & $2.56 \mathrm{e}-08$ \\
\hline 100 & $3 \mathrm{~s}^{2} 3 \mathrm{p}^{2}\left({ }_{2}^{3} \mathrm{P}\right){ }^{3} \mathrm{P} 5 \mathrm{p}^{2} \mathrm{P}_{1 / 2}^{\mathrm{o} / 2}$ & $0.79+0.093 \mathrm{~s}^{2} 3 \mathrm{p}^{2}\left({ }_{0}^{1} \mathrm{~S}\right){ }^{1} \mathrm{~S} 4 \mathrm{p}^{2} \mathrm{P}^{\circ}+0.023 \mathrm{~s}^{2} 3 \mathrm{p}^{2}\left({ }_{2}^{3} \mathrm{P}\right){ }^{3} \mathrm{P} 4 \mathrm{p}^{2} \mathrm{P}^{\circ}$ & 159333 & & $1.15 \mathrm{e}-08$ & $1.51 \mathrm{e}-08$ \\
\hline 101 & $3 \mathrm{~s}^{2} 3 \mathrm{p}^{2}\left({ }_{2}^{3} \mathrm{P}\right){ }^{3} \mathrm{P} 5 \mathrm{p}^{2} \mathrm{P}_{3 / 2}^{\mathrm{o}}$ & $0.80+0.083 \mathrm{~s}^{2} 3 \mathrm{p}^{2}\left({ }_{0}^{1} \mathrm{~S}\right){ }^{1} \mathrm{~S} 4 \mathrm{p}^{2} \mathrm{P}^{\circ}+0.023 \mathrm{~s}^{2} 3 \mathrm{p}^{2}\left({ }_{2}^{3} \mathrm{P}\right){ }^{3} \mathrm{P} 4 \mathrm{p}^{2} \mathrm{P}^{\circ}$ & 159504 & & $1.18 \mathrm{e}-08$ & $1.56 \mathrm{e}-08$ \\
\hline 102 & $3 \mathrm{~s}^{2} \mathrm{~S} 3 \mathrm{p}^{4}\left({ }_{2}^{3} \mathrm{P}\right){ }^{2} \mathrm{P}_{3 / 2}$ & $\mathbf{0 . 1 8}+0.313 \mathrm{~s}^{2} 3 \mathrm{p}^{2}\left({ }_{2}^{3} \mathrm{P}\right){ }^{3} \mathrm{P} 4 \mathrm{~d}^{2} \mathrm{P}+0.233 \mathrm{~s}^{2} 3 \mathrm{p}^{2}\left({ }_{2}^{3} \mathrm{P}\right){ }^{3} \mathrm{P} 4 \mathrm{~d}^{2} \mathrm{D}$ & 159564 & 158828.31 & $1.56 \mathrm{e}-10$ & $1.56 \mathrm{e}-10$ \\
\hline 103 & $3 \mathrm{~s}^{2} 3 \mathrm{p}^{2}\left({ }_{2}^{3} \mathrm{P}\right){ }^{3} \mathrm{P} 4 \mathrm{f}^{4} \mathrm{G}_{5 / 2}^{\circ}$ & $0.49+0.263 \mathrm{~s}^{2} 3 \mathrm{p}^{2}\left({ }_{2}^{3} \mathrm{P}\right){ }^{3} \mathrm{P} 4 \mathrm{f}^{2} \mathrm{D}^{\circ}+0.083 \mathrm{~s}^{2} 3 \mathrm{p}^{2}\left({ }_{2}^{3} \mathrm{P}\right){ }^{3} \mathrm{P} 4 \mathrm{f}^{4} \mathrm{D}^{\circ}$ & 159793 & 160104.11 & $3.24 \mathrm{e}-09$ & $3.25 \mathrm{e}-09$ \\
\hline 104 & $3 \mathrm{~s}^{2} 3 \mathrm{p}^{2}\left({ }_{2}^{3} \mathrm{P}\right){ }^{3} \mathrm{P} 4 \mathrm{f}^{4} \mathrm{D}_{7 / 2}^{\circ}$ & $0.50+0.193 \mathrm{~s}^{2} 3 \mathrm{p}^{2}\left({ }_{2}^{3} \mathrm{P}\right){ }^{3} \mathrm{P} 4 \mathrm{f}^{4} \mathrm{G}^{\circ}+0.133 \mathrm{~s}^{2} 3 \mathrm{p}^{2}\left({ }_{2}^{3} \mathrm{P}\right){ }^{3} \mathrm{P} 4 \mathrm{f}^{2} \mathrm{G}^{\circ}$ & 159818 & 160128.9 & $3.29 \mathrm{e}-09$ & $3.34 \mathrm{e}-09$ \\
\hline 105 & $3 \mathrm{~s}^{2} 3 \mathrm{p}^{2}\left({ }_{2}^{3} \mathrm{P}\right){ }^{3} \mathrm{P} 4 \mathrm{~d}^{2} \mathrm{D}_{5 / 2}$ & $0.71+0.083 \mathrm{~s}^{2} 3 \mathrm{p}^{2}\left({ }_{0}^{1} \mathrm{~S}\right){ }^{1} \mathrm{~S} 3 \mathrm{~d}^{2} \mathrm{D}+0.073 \mathrm{~s}^{2} 3 \mathrm{p}^{2}\left({ }_{2}^{3} \mathrm{P}\right){ }^{3} \mathrm{P} 3 \mathrm{~d}^{2} \mathrm{D}$ & 159843 & 159492.83 & $2.90 \mathrm{e}-10$ & $2.96 \mathrm{e}-10$ \\
\hline 106 & $3 \mathrm{~s}^{2} 3 \mathrm{p}^{2}\left({ }_{2}^{3} \mathrm{P}\right){ }^{3} \mathrm{P} 4 \mathrm{~d}^{2} \mathrm{D}_{3 / 2}$ & $0.47+0.163 \mathrm{~s}^{2} 3 \mathrm{p}^{2}\left({ }_{2}^{3} \mathrm{P}\right){ }^{3} \mathrm{P} 4 \mathrm{~d}^{2} \mathrm{P}+0.093 \mathrm{~s}^{2} \mathrm{~S} 3 \mathrm{p}^{4}\left({ }_{2}^{3} \mathrm{P}\right){ }^{2} \mathrm{P}$ & 159935 & 159495.94 & $2.11 \mathrm{e}-10$ & $2.12 \mathrm{e}-10$ \\
\hline 107 & $3 \mathrm{~s}^{2} 3 \mathrm{p}^{2}\left({ }_{2}^{3} \mathrm{P}\right){ }^{3} \mathrm{P} 4 \mathrm{f}^{2} \mathrm{D}_{5 / 2}^{\circ}$ & $\mathbf{0 . 4 0}+0.413 \mathrm{~s}^{2} 3 \mathrm{p}^{2}\left({ }_{2}^{3} \mathrm{P}\right){ }^{3} \mathrm{P} 4 \mathrm{f}^{4} \mathrm{G}^{\circ}+0.123 \mathrm{~s}^{2} 3 \mathrm{p}^{2}\left({ }_{2}^{3} \mathrm{P}\right){ }^{3} \mathrm{P} 4 \mathrm{f}^{4} \mathrm{D}^{\circ}$ & 159946 & 160258.03 & $3.24 \mathrm{e}-09$ & $3.26 \mathrm{e}-09$ \\
\hline 108 & $3 \mathrm{~s}^{2} 3 \mathrm{p}^{2}\left({ }_{2}^{3} \mathrm{P}\right){ }^{3} \mathrm{P} 4 \mathrm{f}^{4} \mathrm{G}_{7 / 2}^{\circ}$ & $0.40+0.363 \mathrm{~s}^{2} 3 \mathrm{p}^{2}\left({ }_{2}^{3} \mathrm{P}\right){ }^{3} \mathrm{P} 4 \mathrm{f}^{4} \mathrm{D}^{\circ}+0.173 \mathrm{~s}^{2} 3 \mathrm{p}^{2}\left({ }_{2}^{3} \mathrm{P}\right){ }^{3} \mathrm{P} 4 \mathrm{f}^{2} \mathrm{G}^{\circ}$ & 159975 & 160284.24 & $3.26 \mathrm{e}-09$ & $3.30 \mathrm{e}-09$ \\
\hline 109 & $3 \mathrm{~s}^{2} 3 \mathrm{p}^{2}\left({ }_{2}^{2} \mathrm{P}\right){ }^{3} \mathrm{P} 4 \mathrm{~d}^{2} \mathrm{P}_{1 / 2}$ & $0.48+0.273 \mathrm{~s}^{2} \mathrm{~S} 3 \mathrm{p}^{4}\left({ }_{2}^{3} \mathrm{P}\right){ }^{2} \mathrm{P}+0.053 \mathrm{~s}^{2} 3 \mathrm{p}^{2}\left({ }_{2}^{3} \mathrm{P}\right){ }^{3} \mathrm{P} 3 \mathrm{~d}^{2} \mathrm{P}$ & 160132 & 159283.66 & $1.29 \mathrm{e}-10$ & $1.28 \mathrm{e}-10$ \\
\hline 110 & $3 \mathrm{~s}^{2} 3 \mathrm{p}^{2}\left({ }_{2}^{3} \mathrm{P}\right){ }^{3} \mathrm{P} 4 \mathrm{f}^{4} \mathrm{G}_{9 / 2}^{\circ}$ & $0.70+0.153 \mathrm{~s}^{2} 3 \mathrm{p}^{2}\left({ }_{2}^{3} \mathrm{P}\right){ }^{3} \mathrm{P} 4 \mathrm{f}^{2} \mathrm{G}^{\circ}+0.083 \mathrm{~s}^{2} 3 \mathrm{p}^{2}\left({ }_{2}^{3} \mathrm{P}\right){ }^{3} \mathrm{P} 4 \mathrm{f}^{4} \mathrm{~F}^{\circ}$ & 160134 & 160442.67 & $3.23 \mathrm{e}-09$ & $3.21 \mathrm{e}-09$ \\
\hline 111 & $3 \mathrm{~s}^{2} 3 \mathrm{p}^{2}\left({ }_{2}^{3} \mathrm{P}\right){ }^{3} \mathrm{P} 4 \mathrm{f}^{4} \mathrm{D}_{3 / 2}^{\circ}$ & $0.41+0.403 \mathrm{~s}^{2} 3 \mathrm{p}^{2}\left({ }_{2}^{3} \mathrm{P}\right){ }^{3} \mathrm{P} 4 \mathrm{f}^{2} \mathrm{D}^{\circ}+0.123 \mathrm{~s}^{2} 3 \mathrm{p}^{2}\left({ }_{2}^{3} \mathrm{P}\right){ }^{3} \mathrm{P} 4 \mathrm{f}^{4} \mathrm{~F}^{\circ}$ & 160149 & 160462.24 & $3.30 \mathrm{e}-09$ & $3.35 \mathrm{e}-09$ \\
\hline 112 & $3 \mathrm{~s}^{2} 3 \mathrm{p}^{2}\left({ }_{2}^{3} \mathrm{P}\right){ }^{3} \mathrm{P} 4 \mathrm{f}^{4} \mathrm{D}_{5 / 2}^{\mathrm{o}}$ & $0.62+0.203 \mathrm{~s}^{2} 3 \mathrm{p}^{2}\left({ }_{2}^{3} \mathrm{P}\right){ }^{3} \mathrm{P} 4 \mathrm{f}^{2} \mathrm{D}^{\circ}+0.053 \mathrm{~s}^{2} 3 \mathrm{p}^{2}\left({ }_{2}^{3} \mathrm{P}\right){ }^{3} \mathrm{P} 4 \mathrm{f}^{2} \mathrm{~F}^{\circ}$ & 160155 & 160467.51 & $3.29 \mathrm{e}-09$ & $3.37 \mathrm{e}-09$ \\
\hline 113 & $3 \mathrm{~s}^{2} 3 \mathrm{p}^{2}\left({ }_{2}^{3} \mathrm{P}\right){ }^{3} \mathrm{P} 4 \mathrm{f}^{2} \mathrm{G}_{7 / 2}^{\mathrm{o} / 2}$ & $0.58+0.283 \mathrm{~s}^{2} 3 \mathrm{p}^{2}\left({ }_{2}^{3} \mathrm{P}\right){ }^{3} \mathrm{P} 4 \mathrm{f}^{4} \mathrm{G}^{\circ}+0.063 \mathrm{~s}^{2} 3 \mathrm{p}^{2}\left({ }_{2}^{3} \mathrm{P}\right){ }^{3} \mathrm{P} 4 \mathrm{f}^{2} \mathrm{~F}^{\circ}$ & 160173 & 160483.16 & $3.50 \mathrm{e}-09$ & $3.58 \mathrm{e}-09$ \\
\hline 114 & $3 \mathrm{~s}^{2} 3 \mathrm{p}^{2}\left({ }_{2}^{3} \mathrm{P}\right){ }^{3} \mathrm{P} 4 \mathrm{f}^{2} \mathrm{D}_{3 / 2}^{\circ}$ & $0.47+0.463 \mathrm{~s}^{2} 3 \mathrm{p}^{2}\left({ }_{2}^{3} \mathrm{P}\right){ }^{3} \mathrm{P} 4 \mathrm{f}^{4} \mathrm{D}^{\circ}$ & 160425 & 160733.68 & $3.28 \mathrm{e}-09$ & $3.36 \mathrm{e}-09$ \\
\hline 115 & $3 \mathrm{~s}^{2} 3 \mathrm{p}^{2}\left({ }_{2}^{3} \mathrm{P}\right){ }^{3} \mathrm{P} 4 \mathrm{f}^{4} \mathrm{D}_{1 / 2}^{\circ}$ & 0.93 & 160428 & 160733.68 & $3.22 \mathrm{e}-09$ & $3.30 \mathrm{e}-09$ \\
\hline 116 & $3 \mathrm{~s}^{2} 3 \mathrm{p}^{2}\left({ }_{2}^{3} \mathrm{P}\right){ }^{3} \mathrm{P} 4 \mathrm{f}^{4} \mathrm{G}_{11 / 2}^{\mathrm{o}}$ & 0.93 & 160522 & 160828.69 & $3.14 \mathrm{e}-09$ & $3.10 \mathrm{e}-09$ \\
\hline 117 & $3 \mathrm{~s}^{2} 3 \mathrm{p}^{2}\left({ }_{2}^{3} \mathrm{P}\right){ }^{3} \mathrm{P} 4 \mathrm{f}^{2} \mathrm{G}_{9 / 2}^{\circ}$ & $0.75+0.183 \mathrm{~s}^{2} 3 \mathrm{p}^{2}\left({ }_{2}^{3} \mathrm{P}\right){ }^{3} \mathrm{P} 4 \mathrm{f}^{4} \mathrm{G}^{\circ}$ & 160612 & 160920.95 & $3.56 \mathrm{e}-09$ & $3.67 \mathrm{e}-09$ \\
\hline 118 & $3 \mathrm{~s}^{2} 3 \mathrm{p}^{2}\left({ }_{2}^{3} \mathrm{P}\right){ }^{3} \mathrm{P} 4 \mathrm{f}^{4} \mathrm{~F}_{3 / 2}^{\circ}$ & $0.81+0.063 \mathrm{~s}^{2} 3 \mathrm{p}^{2}\left({ }_{2}^{3} \mathrm{P}\right){ }^{3} \mathrm{P} 4 \mathrm{f}^{2} \mathrm{D}^{\circ}+0.063 \mathrm{~s}^{2} 3 \mathrm{p}^{2}\left({ }_{2}^{3} \mathrm{P}\right){ }^{3} \mathrm{P} 4 \mathrm{f}^{4} \mathrm{D}^{\circ}$ & 160888 & 161221.05 & $3.33 \mathrm{e}-09$ & $3.28 \mathrm{e}-09$ \\
\hline
\end{tabular}


Table A.1. continued.

\begin{tabular}{|c|c|c|c|c|c|c|}
\hline 119 & $3 \mathrm{~s}^{2} 3 \mathrm{p}^{2}\left({ }_{2}^{3} \mathrm{P}\right){ }^{3} \mathrm{P} 4 \mathrm{f}^{4} \mathrm{~F}_{5 / 2}^{\circ}$ & $0.70+0.123 \mathrm{~s}^{2} 3 \mathrm{p}^{2}\left({ }_{2}^{3} \mathrm{P}\right){ }^{3} \mathrm{P} 4 \mathrm{f}^{2} \mathrm{~F}^{\circ}+0.103 \mathrm{~s}^{2} 3 \mathrm{p}^{2}\left({ }_{2}^{3} \mathrm{P}\right){ }^{3} \mathrm{P} 4 \mathrm{f}^{4} \mathrm{D}^{\circ}$ & 160919 & 161253.47 & $3.40 \mathrm{e}-09$ & $3.38 \mathrm{e}-09$ \\
\hline 120 & $3 \mathrm{~s}^{2} 3 \mathrm{p}^{2}\left({ }_{2}^{3} \mathrm{P}\right){ }^{3} \mathrm{P} 4 \mathrm{f}^{4} \mathrm{~F}_{7 / 2}^{\circ}$ & $0.82+0.063 \mathrm{~s}^{2} 3 \mathrm{p}^{2}\left({ }_{2}^{3} \mathrm{P}\right){ }^{3} \mathrm{P} 4 \mathrm{f}^{4} \mathrm{D}^{\circ}+0.043 \mathrm{~s}^{2} 3 \mathrm{p}^{2}\left({ }_{2}^{3} \mathrm{P}\right){ }^{3} \mathrm{P} 4 \mathrm{f}^{4} \mathrm{G}^{\circ}$ & 160992 & 161329.65 & $3.32 \mathrm{e}-09$ & $3.27 \mathrm{e}-09$ \\
\hline 121 & $3 \mathrm{~s}^{2} 3 \mathrm{p}^{2}\left({ }_{2}^{3} \mathrm{P}\right){ }^{3} \mathrm{P} 4 \mathrm{f}^{4} \mathrm{~F}_{9 / 2}^{\circ}$ & $0.84+0.053 \mathrm{~s}^{2} 3 \mathrm{p}^{2}\left({ }_{2}^{3} \mathrm{P}\right){ }^{3} \mathrm{P} 4 \mathrm{f}^{4} \mathrm{G}^{\circ}+0.043 \mathrm{~s}^{2} 3 \mathrm{p}^{2}\left({ }_{2}^{3} \mathrm{P}\right){ }^{3} \mathrm{P} 4 \mathrm{f}^{2} \mathrm{G}^{\circ}$ & 161024 & 161360.19 & $3.34 \mathrm{e}-09$ & $3.29 \mathrm{e}-09$ \\
\hline 122 & $3 \mathrm{~s}^{2} 3 \mathrm{p}^{2}\left({ }_{2}^{3} \mathrm{P}\right){ }^{3} \mathrm{P} 4 \mathrm{f}^{2} \mathrm{~F}_{5 / 2}^{\mathrm{o}}$ & $0.72+0.123 \mathrm{~s}^{2} 3 \mathrm{p}^{2}\left({ }_{2}^{3} \mathrm{P}\right){ }^{3} \mathrm{P} 4 \mathrm{f}^{4} \mathrm{~F}^{\circ}+0.073 \mathrm{~s}^{2} 3 \mathrm{p}^{2}\left({ }_{2}^{3} \mathrm{P}\right){ }^{3} \mathrm{P} 4 \mathrm{f}^{2} \mathrm{D}^{\circ}$ & 161059 & 161386.45 & $3.86 \mathrm{e}-09$ & $4.03 \mathrm{e}-09$ \\
\hline 123 & $3 \mathrm{~s}^{2} 3 \mathrm{p}^{2}\left({ }_{2}^{3} \mathrm{P}\right){ }^{3} \mathrm{P} 4 \mathrm{f}^{2} \mathrm{~F}_{7 / 2}^{\circ}$ & $0.85+0.053 \mathrm{~s}^{2} 3 \mathrm{p}^{2}\left({ }_{2}^{3} \mathrm{P}\right){ }^{3} \mathrm{P} 4 \mathrm{f}^{2} \mathrm{G}^{\circ}$ & 161127 & 161454.96 & $4.00 \mathrm{e}-09$ & $4.23 \mathrm{e}-09$ \\
\hline 124 & $3 \mathrm{~s}^{2} 3 \mathrm{p}^{2}\left({ }_{2}^{1} \mathrm{D}\right){ }^{1} \mathrm{D} 5 \mathrm{~s}^{2} \mathrm{D}_{5 / 2}$ & 0.91 & 161595 & 161733.1 & $1.52 \mathrm{e}-09$ & $1.53 \mathrm{e}-09$ \\
\hline 125 & $3 \mathrm{~s}^{2} 3 \mathrm{p}^{2}\left({ }_{2}^{\mathrm{T}} \mathrm{D}\right){ }^{1} \mathrm{D} 5 \mathrm{~s}^{2} \mathrm{D}_{3 / 2}$ & 0.91 & 161603 & 161737.99 & $1.39 \mathrm{e}-09$ & $1.40 \mathrm{e}-09$ \\
\hline 126 & $3 s^{2} S 3 p^{3}\left({ }_{3}^{4} S\right){ }^{5} S 3 d^{6} D_{5 / 2}^{\circ}$ & 0.94 & 164076 & & $1.43 \mathrm{e}-05$ & $1.46 \mathrm{e}-05$ \\
\hline 127 & $3 s^{2} S 3 p^{3}\left({ }_{3}^{4} S\right){ }^{5} S 3 d^{6} D_{3 / 2}^{\circ}$ & 0.94 & 164077 & & $1.08 \mathrm{e}-05$ & $1.05 \mathrm{e}-05$ \\
\hline 128 & $3 s^{2} S 3 p^{3}\left({ }_{3}^{4} S\right){ }^{5} S 3 d^{6} D_{7 / 2}^{\circ}$ & 0.94 & 164080 & & $2.13 \mathrm{e}-05$ & $2.26 \mathrm{e}-05$ \\
\hline 129 & $3 s^{2} S 3 p^{3}\left({ }_{3}^{4} S\right){ }^{5} S 3 d^{6} D_{1 / 2}^{\circ}$ & 0.94 & 164083 & & $9.17 \mathrm{e}-06$ & $8.52 \mathrm{e}-06$ \\
\hline 130 & $3 \mathrm{~s}^{2} \mathrm{~S} 3 \mathrm{p}^{3}\left({ }_{3}^{4} \mathrm{~S}\right){ }^{5} \mathrm{~S} 3 \mathrm{~d}^{6} \mathrm{D}_{9 / 2}^{\circ}$ & 0.94 & 164088 & & $2.66 \mathrm{e}-05$ & $2.30 \mathrm{e}-05$ \\
\hline 131 & $3 \mathrm{~s}^{2} 3 \mathrm{p}^{2}\left({ }_{2}^{1} \mathrm{D}\right){ }^{1} \mathrm{D} 4 \mathrm{~d}^{2} \mathrm{G}_{7 / 2}$ & 0.91 & 164185 & 164181.17 & $4.17 \mathrm{e}-09$ & $4.23 \mathrm{e}-09$ \\
\hline 132 & $3 \mathrm{~s}^{2} 3 \mathrm{p}^{2}\left({ }_{2}^{1} \mathrm{D}\right){ }^{1} \mathrm{D} 4 \mathrm{~d}^{2} \mathrm{G}_{9 / 2}$ & 0.92 & 164210 & 164268.79 & $4.56 \mathrm{e}-09$ & $4.62 \mathrm{e}-09$ \\
\hline 133 & $3 \mathrm{~s}^{2} 3 \mathrm{p}^{2}\left({ }_{2}^{1} \mathrm{D}\right){ }^{1} \mathrm{D} 4 \mathrm{~d}^{2} \mathrm{~F}_{7 / 2}$ & $0.75+0.093 \mathrm{~s}^{2} 3 \mathrm{p}^{2}\left({ }_{2}^{3} \mathrm{P}\right){ }^{3} \mathrm{P} 4 \mathrm{~d}^{2} \mathrm{~F}+0.033 \mathrm{~s}^{2} 3 \mathrm{p}^{2}\left({ }_{2}^{3} \mathrm{P}\right){ }^{3} \mathrm{P} 5 \mathrm{~d}^{2} \mathrm{~F}$ & 165008 & 164337.61 & $4.36 \mathrm{e}-10$ & $4.43 \mathrm{e}-10$ \\
\hline 134 & $3 \mathrm{~s}^{2} 3 \mathrm{p}^{2}\left({ }_{2}^{\mathrm{T}} \mathrm{D}\right){ }^{1} \mathrm{D} 4 \mathrm{~d}^{2} \mathrm{~F}_{5 / 2}$ & $0.76+0.103 \mathrm{~s}^{2} 3 \mathrm{p}^{2}\left({ }_{2}^{3} \mathrm{P}\right){ }^{3} \mathrm{P} 4 \mathrm{~d}^{2} \mathrm{~F}+0.033 \mathrm{~s}^{2} 3 \mathrm{p}^{2}\left({ }_{2}^{3} \mathrm{P}\right){ }^{3} \mathrm{P} 5 \mathrm{~d}^{2} \mathrm{~F}$ & 165084 & 164232.36 & $4.31 \mathrm{e}-10$ & $4.38 \mathrm{e}-10$ \\
\hline
\end{tabular}

Table A.2. Wave function composition (up to three $L S$ components with a contribution $>0.02$ of the total wave function) in $L S$-coupling and energy levels (in $\mathrm{cm}^{-1}$ ) for $\mathrm{Cl}$ III.

\begin{tabular}{|c|c|c|c|c|c|c|}
\hline No. & State & $L S$-composition & $E_{\mathrm{RCI}(\mathrm{CV})}$ & $E_{\mathrm{NIST}}$ & $\tau_{1}$ & $\tau_{\mathrm{v}}$ \\
\hline 1 & $3 s^{2} 3 p^{3}\left({ }_{3}^{4} S\right){ }^{4} S_{3 / 2}^{\circ}$ & $0.94+0.033 \mathrm{~s}^{2} \mathrm{~S} 3 \mathrm{p}^{3}\left({ }_{3}^{2} \mathrm{D}\right){ }^{3} \mathrm{D} 3 \mathrm{~d}^{4} \mathrm{~S}^{\circ}$ & 0 & 0 & & \\
\hline 2 & $3 \mathrm{~s}^{2} 3 \mathrm{p}^{3}\left({ }_{3}^{2} \mathrm{D}\right){ }^{2} \mathrm{D}_{3 / 2}^{\mathrm{o}}$ & 0.93 & 18286 & 18052.46 & $1.82 \mathrm{e}+02$ & $1.83 \mathrm{e}+02$ \\
\hline 3 & $3 \mathrm{~s}^{2} 3 \mathrm{p}^{3}\left({ }_{3}^{2} \mathrm{D}\right)^{2} \mathrm{D}_{5 / 2}^{\circ}$ & 0.93 & 18345 & 18118.43 & $1.27 \mathrm{e}+03$ & $1.35 \mathrm{e}+03$ \\
\hline 4 & $3 \mathrm{~s}^{2} 3 \mathrm{p}^{3}\left({ }_{1}^{2} \mathrm{P}\right){ }^{2} \mathrm{P}_{1 / 2}^{\mathrm{o} / 2}$ & $0.92+0.023 \mathrm{p}^{5}{ }^{2} \mathrm{P}^{\circ}$ & 30117 & 29813.92 & $1.38 \mathrm{e}+00$ & $1.41 \mathrm{e}+00$ \\
\hline 5 & $3 \mathrm{~s}^{2} 3 \mathrm{p}^{3}\left({ }_{1}^{2} \mathrm{P}\right){ }^{2} \mathrm{P}_{3 / 2}^{\mathrm{o}}$ & $0.91+0.023 \mathrm{p}^{5}{ }^{2} \mathrm{P}^{\circ}$ & 30200 & 29906.45 & $6.91 \mathrm{e}-01$ & $6.96 \mathrm{e}-01$ \\
\hline 6 & $3 \mathrm{~s}^{2} \mathrm{~S} 3 \mathrm{p}^{4}\left({ }_{2}^{3} \mathrm{P}\right){ }^{4} \mathrm{P}_{5 / 2}^{3 / 2}$ & $0.83+0.123 \mathrm{~s}^{2} 3 \mathrm{p}^{2}\left({ }_{2}^{3} \mathrm{P}\right){ }^{3} \mathrm{P} 3 \mathrm{~d}^{4} \mathrm{P}$ & 98508 & 98520.34 & $7.47 \mathrm{e}-09$ & $7.17 \mathrm{e}-09$ \\
\hline 7 & $3 \mathrm{~s}^{2} \mathrm{~S} 3 \mathrm{p}^{4}\left({ }_{2}^{3} \mathrm{P}\right){ }^{4} \mathrm{P}_{3 / 2}$ & $\left.0.83+0.133 \mathrm{~s}^{2} 3 \mathrm{p}^{2}{ }_{2}^{3} \mathrm{P}\right){ }^{3} \mathrm{P} 3 \mathrm{~d}^{4} \mathrm{P}$ & 99114 & 99131.4 & $7.37 \mathrm{e}-09$ & $7.10 \mathrm{e}-09$ \\
\hline 8 & $\left.3 \mathrm{~s}^{2} \mathrm{~S} 3 \mathrm{p}^{4}{ }_{2}^{3} \mathrm{P}\right){ }^{4} \mathrm{P}_{1 / 2}$ & $0.83+0.133 \mathrm{~s}^{2} 3 \mathrm{p}^{2}\left({ }_{2}^{3} \mathrm{P}\right){ }^{3} \mathrm{P} 3 \mathrm{~d}^{4} \mathrm{P}$ & 99462 & 99475.22 & $7.31 \mathrm{e}-09$ & $7.05 \mathrm{e}-09$ \\
\hline 9 & $3 s^{2} S 3 p^{4}\left({ }_{2}^{1} D\right)^{2} D_{3 / 2}$ & $0.67+0.233 \mathrm{~s}^{2} 3 \mathrm{p}^{2}\left({ }_{2}^{1} \mathrm{D}\right){ }^{1} \mathrm{D} 3 \mathrm{~d}^{2} \mathrm{D}+0.033 \mathrm{~s}^{2} 3 \mathrm{p}^{2}\left({ }_{0}^{1} \mathrm{~S}\right){ }^{1} \mathrm{~S} 3 \mathrm{~d}^{2} \mathrm{D}$ & 122392 & 122131.66 & $4.37 \mathrm{e}-09$ & $4.21 \mathrm{e}-09$ \\
\hline 10 & $3 \mathrm{~s}^{2} \mathrm{~S} 3 \mathrm{p}^{4}\left({ }_{2}^{1} \mathrm{D}\right){ }^{2} \mathrm{D}_{5 / 2}$ & $0.67+0.233 \mathrm{~s}^{2} 3 \mathrm{p}^{2}\left({ }_{2}^{1} \mathrm{D}\right){ }^{1} \mathrm{D} 3 \mathrm{~d}^{2} \mathrm{D}+0.033 \mathrm{~s}^{2} 3 \mathrm{p}^{2}\left({ }_{0}^{1} \mathrm{~S}\right){ }^{1} \mathrm{~S} 3 \mathrm{~d}^{2} \mathrm{D}$ & 122443 & 122179.8 & $4.48 \mathrm{e}-09$ & $4.32 \mathrm{e}-09$ \\
\hline 11 & $3 \mathrm{~s}^{2} 3 \mathrm{p}^{2}\left({ }_{2}^{3} \mathrm{P}\right){ }^{3} \mathrm{P} 3 \mathrm{~d}^{2} \mathrm{P}_{3 / 2}$ & $0.60+0.263 \mathrm{~s}^{2} \mathrm{~S} 3 \mathrm{p}^{4}\left({ }_{2}^{3} \mathrm{P}\right){ }^{2} \mathrm{P}+0.073 \mathrm{~s}^{2} 3 \mathrm{p}^{2}\left({ }_{2}^{1} \mathrm{D}\right){ }^{1} \mathrm{D} 3 \mathrm{~d}^{2} \mathrm{P}$ & 137180 & & $2.62 \mathrm{e}-09$ & $2.55 \mathrm{e}-09$ \\
\hline 12 & $3 \mathrm{~s}^{2} 3 \mathrm{p}^{2}\left({ }_{2}^{3} \mathrm{P}\right){ }^{3} \mathrm{P} 3 \mathrm{~d}^{2} \mathrm{P}_{1 / 2}$ & $0.60+0.263 \mathrm{~s}^{2} \mathrm{~S} 3 \mathrm{p}^{4}\left({ }_{2}^{3} \mathrm{P}\right){ }^{2} \mathrm{P}+0.073 \mathrm{~s}^{2} 3 \mathrm{p}^{2}\left({ }_{2}^{1} \mathrm{D}\right){ }^{1} \mathrm{D} 3 \mathrm{~d}^{2} \mathrm{P}$ & 137920 & & $2.52 \mathrm{e}-09$ & $2.46 \mathrm{e}-09$ \\
\hline 13 & $3 \mathrm{~s}^{2} 3 \mathrm{p}^{2}\left({ }_{2}^{3} \mathrm{P}\right){ }^{3} \mathrm{P} 3 \mathrm{~d}^{4} \mathrm{~F}_{3 / 2}$ & 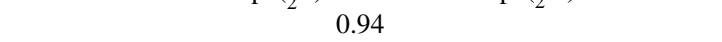 & 146611 & 146521.72 & $1.12 \mathrm{e}-06$ & $1.08 \mathrm{e}-06$ \\
\hline 14 & $3 \mathrm{~s}^{2} 3 \mathrm{p}^{2}\left({ }_{2}^{3} \mathrm{P}\right){ }^{3} \mathrm{P} 3 \mathrm{~d}^{4} \mathrm{~F}_{5 / 2}$ & 0.94 & 146835 & 146746.99 & $1.28 \mathrm{e}-06$ & $1.29 \mathrm{e}-06$ \\
\hline 15 & $3 \mathrm{~s}^{2} 3 \mathrm{p}^{2}\left({ }_{2}^{3} \mathrm{P}\right){ }^{3} \mathrm{P} 3 \mathrm{~d}^{4} \mathrm{~F}_{7 / 2}$ & 0.94 & 147165 & 147069.57 & $1.94 \mathrm{e}-06$ & $1.99 \mathrm{e}-06$ \\
\hline 16 & $3 \mathrm{~s}^{2} 3 \mathrm{p}^{2}\left({ }_{2}^{3} \mathrm{P}\right){ }^{3} \mathrm{P} 3 \mathrm{~d}^{4} \mathrm{~F}_{9 / 2}$ & 0.94 & 147600 & 147494.54 & $3.10 \mathrm{e}-01$ & $2.98 \mathrm{e}-01$ \\
\hline 17 & $3 \mathrm{~s}^{2} \mathrm{~S} \mathrm{3} \mathrm{p}^{4}\left({ }_{0}^{1} \mathrm{~S}\right){ }^{2} \mathrm{~S}_{1 / 2}$ & $0.67+0.273 \mathrm{~s}^{2} 3 \mathrm{p}^{2}\left({ }_{2}^{1} \mathrm{D}\right){ }^{1} \mathrm{D} 3 \mathrm{~d}^{2} \mathrm{~S}$ & 150041 & & $1.27 \mathrm{e}-09$ & $1.23 \mathrm{e}-09$ \\
\hline 18 & $3 s^{2} 3 p^{2}\left({ }_{2}^{3} \mathrm{P}\right){ }^{3} \mathrm{P} 3 \mathrm{~d}^{4} \mathrm{D}_{5 / 2}$ & $0.58+0.203 \mathrm{~s}^{2} 3 \mathrm{p}^{2}\left({ }_{2}^{1} \mathrm{D}\right){ }^{1} \mathrm{D} 3 \mathrm{~d}^{2} \mathrm{~F}+0.153 \mathrm{~s}^{2} 3 \mathrm{p}^{2}\left({ }_{2}^{3} \mathrm{P}\right){ }^{3} \mathrm{P} 3 \mathrm{~d}^{2} \mathrm{~F}$ & 151972 & 151846.4 & $1.55 \mathrm{e}-07$ & $1.54 \mathrm{e}-07$ \\
\hline 19 & $3 s^{2} 3 p^{2}\left({ }_{2}^{3} \mathrm{P}\right){ }^{3} \mathrm{P} 3 \mathrm{~d}^{4} \mathrm{D}_{1 / 2}$ & 0.93 & 151979 & 151944.55 & $4.10 \mathrm{e}-07$ & $4.03 e-07$ \\
\hline 20 & $3 s^{2} 3 p^{2}\left({ }_{2}^{3} \mathrm{P}\right){ }^{3} \mathrm{P} 3 \mathrm{~d}^{4} \mathrm{D}_{3 / 2}$ & 0.93 & 152038 & 151878.06 & $2.62 \mathrm{e}-07$ & $2.59 \mathrm{e}-07$ \\
\hline 21 & $3 s^{2} 3 p^{2}\left({ }_{2}^{3} \mathrm{P}\right){ }^{3} \mathrm{P} 3 \mathrm{~d}^{4} \mathrm{D}_{7 / 2}$ & $0.71+0.133 \mathrm{~s}^{2} 3 \mathrm{p}^{2}\left({ }_{2}^{1} \mathrm{D}\right){ }^{1} \mathrm{D} 3 \mathrm{~d}^{2} \mathrm{~F}+0.093 \mathrm{~s}^{2} 3 \mathrm{p}^{2}\left({ }_{2}^{3} \mathrm{P}\right){ }^{3} \mathrm{P} 3 \mathrm{~d}^{2} \mathrm{~F}$ & 152072 & 151951.64 & $1.22 \mathrm{e}-06$ & $1.16 \mathrm{e}-06$ \\
\hline 22 & $3 \mathrm{~s}^{2} 3 \mathrm{p}^{2}\left({ }_{2}^{1} \mathrm{D}\right){ }^{1} \mathrm{D} 3 \mathrm{~d}^{2} \mathrm{~F}_{5 / 2}$ & $\mathbf{0 . 3 3}+0.353 \mathrm{~s}^{2} 3 \mathrm{p}^{2}\left({ }_{2}^{3} \mathrm{P}\right){ }^{3} \mathrm{P} 3 \mathrm{~d}^{4} \mathrm{D}+0.253 \mathrm{~s}^{2} 3 \mathrm{p}^{2}\left({ }_{2}^{3} \mathrm{P}\right){ }^{3} \mathrm{P} 3 \mathrm{~d}^{2} \mathrm{~F}$ & 152458 & & $9.59 \mathrm{e}-08$ & $9.52 \mathrm{e}-08$ \\
\hline 23 & $3 \mathrm{~s}^{2} 3 \mathrm{p}^{2}\left({ }_{2}^{1} \mathrm{D}\right){ }^{1} \mathrm{D} 3 \mathrm{~d}^{2} \mathrm{~F}_{7 / 2}$ & $0.41+0.303 \mathrm{~s}^{2} 3 \mathrm{p}^{2}\left({ }_{2}^{3} \mathrm{P}\right){ }^{3} \mathrm{P} 3 \mathrm{~d}^{2} \mathrm{~F}+0.223 \mathrm{~s}^{2} 3 \mathrm{p}^{2}\left({ }_{2}^{3} \mathrm{P}\right){ }^{3} \mathrm{P} 3 \mathrm{~d}^{4} \mathrm{D}$ & 153210 & & $1.57 \mathrm{e}-07$ & $1.53 \mathrm{e}-07$ \\
\hline 24 & $3 \mathrm{~s}^{2} 3 \mathrm{p}^{2}\left({ }_{2}^{1} \mathrm{D}\right){ }^{1} \mathrm{D} 3 \mathrm{~d}^{2} \mathrm{G}_{7 / 2}$ & 0.93 & 169142 & & $3.48 \mathrm{e}-07$ & $3.50 \mathrm{e}-07$ \\
\hline 25 & $3 \mathrm{~s}^{2} 3 \mathrm{p}^{2}\left({ }_{2}^{1} \mathrm{D}\right){ }^{1} \mathrm{D} 3 \mathrm{~d}^{2} \mathrm{G}_{9 / 2}$ & 0.93 & 169202 & & $2.05 \mathrm{e}-01$ & $2.06 \mathrm{e}-01$ \\
\hline 26 & $3 \mathrm{~s}^{2} 3 \mathrm{p}^{2}\left({ }_{2}^{3} \mathrm{P}\right){ }^{3} \mathrm{P} 4 \mathrm{~s}^{4} \mathrm{P}_{1 / 2}$ & $0.84+0.083 \mathrm{~s}^{2} 3 \mathrm{p}^{2}\left({ }_{2}^{3} \mathrm{P}\right){ }^{3} \mathrm{P} 3 \mathrm{~d}{ }^{4} \mathrm{P}$ & 173576 & 173735.28 & $1.42 \mathrm{e}-10$ & $1.41 \mathrm{e}-10$ \\
\hline 27 & $3 \mathrm{~s}^{2} 3 \mathrm{p}^{2}\left({ }_{2}^{3} \mathrm{P}\right){ }^{3} \mathrm{P} 4 \mathrm{~s}^{4} \mathrm{P}_{3 / 2}$ & $0.82+0.103 \mathrm{~s}^{2} 3 \mathrm{p}^{2}\left({ }_{2}^{3} \mathrm{P}\right){ }^{3} \mathrm{P} 3 \mathrm{~d}^{4} \mathrm{P}$ & 173935 & 174093.02 & $1.28 \mathrm{e}-10$ & $1.28 \mathrm{e}-10$ \\
\hline 28 & $3 s^{2} 3 p^{2}\left({ }_{2}^{3} \mathrm{P}\right){ }^{3} \mathrm{P} 4 \mathrm{~s}^{4} \mathrm{P}_{5 / 2}$ & $0.78+0.133 \mathrm{~s}^{2} 3 \mathrm{p}^{2}\left({ }_{2}^{3} \mathrm{P}\right){ }^{3} \mathrm{P} 3 \mathrm{~d}^{4} \mathrm{P}$ & 174478 & 174611.76 & $1.08 \mathrm{e}-10$ & $1.08 \mathrm{e}-10$ \\
\hline 29 & $3 \mathrm{~s}^{2} 3 \mathrm{p}^{2}\left({ }_{2}^{3} \mathrm{P}\right){ }^{3} \mathrm{P} 4 \mathrm{~s}^{2} \mathrm{P}_{1 / 2}$ & $0.87+0.023 \mathrm{~s}^{2} \mathrm{~S} 3 \mathrm{p}^{4}\left({ }_{2}^{3} \mathrm{P}\right){ }^{2} \mathrm{P}+0.023 \mathrm{~s}^{2} 3 \mathrm{p}^{2}\left({ }_{2}^{1} \mathrm{D}\right){ }^{1} \mathrm{D} 3 \mathrm{~d}^{2} \mathrm{P}$ & 178291 & 178369.14 & $1.14 \mathrm{e}-10$ & $1.12 \mathrm{e}-10$ \\
\hline 30 & $3 \mathrm{~s}^{2} 3 \mathrm{p}^{2}\left({ }_{2}^{3} \mathrm{P}\right){ }^{3} \mathrm{P} 4 \mathrm{~s}^{2} \mathrm{P}_{3 / 2}$ & $0.84+0.043 \mathrm{~s}^{2} \mathrm{~S} 3 \mathrm{p}^{4}\left({ }_{2}^{3} \mathrm{P}\right){ }^{2} \mathrm{P}+0.033 \mathrm{~s}^{2} 3 \mathrm{p}^{2}\left({ }_{2}^{1} \mathrm{D}\right){ }^{1} \mathrm{D} 3 \mathrm{~d}^{2} \mathrm{P}$ & 179006 & 179075.2 & $1.05 \mathrm{e}-10$ & $1.04 \mathrm{e}-10$ \\
\hline 31 & $3 s^{2} 3 p^{2}\left({ }_{2}^{2} \mathrm{P}\right){ }^{3} \mathrm{P} 3 \mathrm{~d}^{4} \mathrm{P}_{5 / 2}$ & $0.67+0.153 \mathrm{~s}^{2} 3 \mathrm{p}^{2}\left({ }_{2}^{3} \mathrm{P}\right){ }^{3} \mathrm{P} 4 \mathrm{~s}{ }^{4} \mathrm{P}+0.103 \mathrm{~s}^{2} \mathrm{~S} 3 \mathrm{p}^{4}\left({ }_{2}^{3} \mathrm{P}\right){ }^{4} \mathrm{P}$ & 179918 & 179493.96 & $7.06 \mathrm{e}-11$ & $7.02 \mathrm{e}-11$ \\
\hline 32 & $3 \mathrm{~s}^{2} 3 \mathrm{p}^{2}\left({ }_{2}^{3} \mathrm{P}\right){ }^{3} \mathrm{P} 3 \mathrm{~d}^{4} \mathrm{P}_{3 / 2}$ & $0.70+0.113 \mathrm{~s}^{2} 3 \mathrm{p}^{2}\left({ }_{2}^{3} \mathrm{P}\right){ }^{3} \mathrm{P} 4 \mathrm{~s}{ }^{4} \mathrm{P}+0.103 \mathrm{~s}^{2} \mathrm{~S} 3 \mathrm{p}^{4}\left({ }_{2}^{3} \mathrm{P}\right){ }^{4} \mathrm{P}$ & 180105 & 179662.15 & $6.38 \mathrm{e}-11$ & $6.34 \mathrm{e}-11$ \\
\hline 33 & $3 s^{2} 3 p^{2}\left({ }_{2}^{3} \mathrm{P}\right){ }^{3} \mathrm{P} 3 \mathrm{~d}^{4} \mathrm{P}_{1 / 2}$ & $0.72+0.113 \mathrm{~s}^{2} \mathrm{~S} 3 \mathrm{p}^{4}\left({ }_{2}^{3} \mathrm{P}\right){ }^{4} \mathrm{P}+0.093 \mathrm{~s}^{2} 3 \mathrm{p}^{2}\left({ }_{2}^{3} \mathrm{P}\right){ }^{3} \mathrm{P} 4 \mathrm{~s}^{4} \mathrm{P}$ & 180253 & 179780.84 & $6.05 \mathrm{e}-11$ & $6.01 \mathrm{e}-11$ \\
\hline 34 & $3 \mathrm{~s}^{2} 3 \mathrm{p}^{2}\left({ }_{2}^{3} \mathrm{P}\right){ }^{3} \mathrm{P} 3 \mathrm{~d}^{2} \mathrm{D}_{3 / 2}$ & $0.50+0.383 \mathrm{~s}^{2} 3 \mathrm{p}^{2}\left({ }_{0}^{1} \mathrm{~S}\right){ }^{1} \mathrm{~S} 3 \mathrm{~d}^{2} \mathrm{D}$ & 182507 & 182075.74 & $4.32 \mathrm{e}-10$ & $4.29 \mathrm{e}-10$ \\
\hline 35 & $3 \mathrm{~s}^{2} 3 \mathrm{p}^{2}\left({ }_{2}^{3} \mathrm{P}\right){ }^{3} \mathrm{P} 3 \mathrm{~d}^{2} \mathrm{D}_{5 / 2}$ & $0.51+0.383 \mathrm{~s}^{2} 3 \mathrm{p}^{2}\left({ }_{0}^{1} \mathrm{~S}\right){ }^{1} \mathrm{~S} 3 \mathrm{~d}^{2} \mathrm{D}+0.023 \mathrm{~s}^{2} \mathrm{~S} 3 \mathrm{p}^{4}\left({ }_{2}^{1} \mathrm{D}\right){ }^{2} \mathrm{D}$ & 183470 & 183041.91 & $3.77 \mathrm{e}-10$ & $3.73 \mathrm{e}-10$ \\
\hline 36 & $3 s^{2} 3 p^{2}\left({ }_{2}^{1} \mathrm{D}\right){ }^{1} \mathrm{D} 3 \mathrm{~d}^{2} \mathrm{P}_{3 / 2}$ & $0.49+0.333 \mathrm{~s}^{2} \mathrm{~S} 3 \mathrm{p}^{4}\left({ }_{2}^{3} \mathrm{P}\right){ }^{2} \mathrm{P}+0.073 \mathrm{~s}^{2} 3 \mathrm{p}^{2}\left({ }_{2}^{3} \mathrm{P}\right){ }^{3} \mathrm{P} 4 \mathrm{~s}^{2} \mathrm{P}$ & 186809 & 185837.68 & $1.03 \mathrm{e}-10$ & $1.01 \mathrm{e}-10$ \\
\hline 37 & $3 \mathrm{~s}^{2} 3 \mathrm{p}^{2}\left({ }_{2}^{1} \mathrm{D}\right){ }^{1} \mathrm{D} 3 \mathrm{~d}^{2} \mathrm{P}_{1 / 2}$ & $0.54+0.323 \mathrm{~s}^{2} \mathrm{~S} 3 \mathrm{p}^{4}\left({ }_{2}^{3} \mathrm{P}\right){ }^{2} \mathrm{P}+0.053 \mathrm{~s}^{2} 3 \mathrm{p}^{2}\left({ }_{2}^{3} \mathrm{P}\right){ }^{3} \mathrm{P} 4 \mathrm{~s}^{2} \mathrm{P}$ & 187208 & 186219.67 & $1.00 \mathrm{e}-10$ & $9.91 \mathrm{e}-11$ \\
\hline
\end{tabular}

Notes. Energy levels are given relative to the ground state. (Level in NIST column flagged "?" means that level/line may not be real; "[]" means that level was determined by interpolation or extrapolation of known experimental values or by semiempirical calculation.). The states 45 and 46 are indicated in bold. The labels for these levels were not assigned with largest contribution to the composition. 
Table A.2. continued.

\begin{tabular}{|c|c|c|c|c|c|c|}
\hline 38 & $3 \mathrm{~s}^{2} 3 \mathrm{p}^{2}\left({ }_{2}^{1} \mathrm{D}\right){ }^{1} \mathrm{D} 4 \mathrm{~s}^{2} \mathrm{D}_{5 / 2}$ & $0.83+0.073 \mathrm{~s}^{2} 3 \mathrm{p}^{2}\left({ }_{2}^{1} \mathrm{D}\right){ }^{1} \mathrm{D} 3 \mathrm{~d}^{2} \mathrm{D}$ & 188428 & 188389.53 & $1.25 \mathrm{e}-10$ & $1.24 \mathrm{e}-10$ \\
\hline 39 & $3 s^{2} 3 p^{2}\left({ }_{2}^{1} \mathrm{D}\right){ }^{1} \mathrm{D} 4 \mathrm{~s}^{2} \mathrm{D}_{3 / 2}$ & $0.82+0.063 \mathrm{~s}^{2} 3 \mathrm{p}^{2}\left({ }_{2}^{1} \mathrm{D}\right){ }^{1} \mathrm{D} 3 \mathrm{~d}^{2} \mathrm{D}$ & 188490 & 188447.52 & $1.27 \mathrm{e}-10$ & $1.27 \mathrm{e}-10$ \\
\hline 40 & $3 \mathrm{~s}^{2} 3 \mathrm{p}^{2}\left({ }_{2}^{1} \mathrm{D}\right){ }^{1} \mathrm{D} 3 \mathrm{~d}^{2} \mathrm{D}_{5 / 2}$ & $0.49+0.133 \mathrm{~s}^{2} \mathrm{~S} 3 \mathrm{p}^{4}\left({ }_{2}^{1} \mathrm{D}\right){ }^{2} \mathrm{D}+0.093 \mathrm{~s}^{2} 3 \mathrm{p}^{2}\left({ }_{2}^{1} \mathrm{D}\right){ }^{1} \mathrm{D} 4 \mathrm{~s}^{2} \mathrm{D}$ & 195683 & 194958.79 & $8.41 \mathrm{e}-11$ & $8.33 e-11$ \\
\hline 41 & $3 \mathrm{~s}^{2} 3 \mathrm{p}^{2}\left({ }_{2}^{1} \mathrm{D}\right){ }^{1} \mathrm{D} 3 \mathrm{~d}^{2} \mathrm{D}_{3 / 2}$ & $0.54+0.153 \mathrm{~s}^{2} \mathrm{~S} 3 \mathrm{p}^{4}\left({ }_{2}^{1} \mathrm{D}\right){ }^{2} \mathrm{D}+0.093 \mathrm{~s}^{2} 3 \mathrm{p}^{2}\left({ }_{2}^{1} \mathrm{D}\right){ }^{1} \mathrm{D} 4 \mathrm{~s}^{2} \mathrm{D}$ & 196011 & 195267.76 & $8.02 \mathrm{e}-11$ & $7.94 \mathrm{e}-11$ \\
\hline 42 & $3 \mathrm{~s}^{2} 3 \mathrm{p}^{2}\left({ }_{2}^{3} \mathrm{P}\right){ }^{3} \mathrm{P} 3 \mathrm{~d}^{2} \mathrm{~F}_{5 / 2}$ & $0.48+0.363 \mathrm{~s}^{2} 3 \mathrm{p}^{2}\left({ }_{2}^{1} \mathrm{D}\right){ }^{1} \mathrm{D} 3 \mathrm{~d}^{2} \mathrm{~F}+0.033 \mathrm{~s}^{2} 3 \mathrm{p}^{2}\left({ }_{2}^{3} \mathrm{P}\right){ }^{3} \mathrm{P} 4 \mathrm{~d}^{2} \mathrm{~F}$ & 196688 & 196137.22 & $4.24 \mathrm{e}-11$ & $4.23 \mathrm{e}-11$ \\
\hline 43 & $3 \mathrm{~s}^{2} 3 \mathrm{p}^{2}\left({ }_{2}^{2} \mathrm{P}\right){ }^{3} \mathrm{P} 3 \mathrm{~d}^{2} \mathrm{~F}_{7 / 2}$ & $0.50+0.373 \mathrm{~s}^{2} 3 \mathrm{p}^{2}\left({ }_{2}^{1} \mathrm{D}\right){ }^{1} \mathrm{D} 3 \mathrm{~d}^{2} \mathrm{~F}+0.033 \mathrm{~s}^{2} 3 \mathrm{p}^{2}\left({ }_{2}^{3} \mathrm{P}\right){ }^{3} \mathrm{P} 4 \mathrm{~d}^{2} \mathrm{~F}$ & 196724 & 196153.36 & $4.18 \mathrm{e}-11$ & $4.18 \mathrm{e}-11$ \\
\hline 44 & $3 \mathrm{~s}^{2} 3 \mathrm{p}^{2}\left({ }_{2}^{3} \mathrm{P}\right){ }^{3} \mathrm{P} 4 \mathrm{p}^{2} \mathrm{~S}_{1 / 2}^{\circ}$ & 0.92 & 197580 & & $2.85 \mathrm{e}-09$ & $2.87 \mathrm{e}-09$ \\
\hline 45 & $3 \mathrm{~s}^{2} \mathrm{~S} 3 \mathrm{p}^{4}\left({ }_{2}^{3} \mathrm{P}\right){ }^{2} \mathrm{P}_{1 / 2}$ & $\mathbf{0 . 2 7}+0.303 \mathrm{~s}^{2} 3 \mathrm{p}^{2}\left({ }_{2}^{3} \mathrm{P}\right){ }^{3} \mathrm{P} 3 \mathrm{~d}^{2} \mathrm{P}+0.273 \mathrm{~s}^{2} 3 \mathrm{p}^{2}\left({ }_{2}^{1} \mathrm{D}\right){ }^{1} \mathrm{D} 3 \mathrm{~d}^{2} \mathrm{P}$ & 199748 & 198835.25 & $5.00 \mathrm{e}-11$ & $4.94 \mathrm{e}-11$ \\
\hline 46 & $3 \mathrm{~s}^{2} \mathrm{~S} 3 \mathrm{p}^{4}\left({ }_{2}^{3} \mathrm{P}\right){ }^{2} \mathrm{P}_{3 / 2}$ & $\mathbf{0 . 2 5}+0.313 \mathrm{~s}^{2} 3 \mathrm{p}^{2}\left({ }_{2}^{1} \mathrm{D}\right){ }^{1} \mathrm{D} 3 \mathrm{~d}^{2} \mathrm{P}+0.303 \mathrm{~s}^{2} 3 \mathrm{p}^{2}\left({ }_{2}^{2} \mathrm{P}\right){ }^{3} \mathrm{P} 3 \mathrm{~d}^{2} \mathrm{P}$ & 199855 & 198983.79 & $5.08 \mathrm{e}-11$ & $5.02 \mathrm{e}-11$ \\
\hline 47 & $3 \mathrm{~s}^{2} 3 \mathrm{p}^{2}\left({ }_{2}^{3} \mathrm{P}\right){ }^{3} \mathrm{P} 4 \mathrm{p}^{4} \mathrm{D}_{1 / 2}^{\circ}$ & 0.92 & 200890 & 201072.41 & $1.77 \mathrm{e}-09$ & $1.80 \mathrm{e}-09$ \\
\hline 48 & $3 \mathrm{~s}^{2} 3 \mathrm{p}^{2}\left({ }_{2}^{2} \mathrm{P}\right){ }^{3} \mathrm{P} 4 \mathrm{p}^{4} \mathrm{D}_{3 / 2}^{\circ}$ & 0.92 & 201139 & 201330.92 & $1.77 \mathrm{e}-09$ & $1.79 \mathrm{e}-09$ \\
\hline 49 & $3 s^{2} 3 p^{2}\left({ }_{2}^{1} D\right){ }^{1} D 3 d^{2} S_{1 / 2}$ & $0.45+0.263 \mathrm{~s}^{2} 3 \mathrm{p}^{2}\left({ }_{0}^{1} \mathrm{~S}\right){ }^{1} \mathrm{~S} 4 \mathrm{~s}^{2} \mathrm{~S}+0.153 \mathrm{~s}^{2} \mathrm{~S} 3 \mathrm{p}^{4}\left({ }_{0}^{1} \mathrm{~S}\right)^{2} \mathrm{~S}$ & 201480 & & $5.29 \mathrm{e}-11$ & $5.24 \mathrm{e}-11$ \\
\hline 50 & $3 \mathrm{~s}^{2} 3 \mathrm{p}^{2}\left({ }_{2}^{3} \mathrm{P}\right){ }^{3} \mathrm{P} 4 \mathrm{p}^{4} \mathrm{D}_{5 / 2}^{\circ}$ & 0.92 & 201568 & 201763.86 & $1.76 \mathrm{e}-09$ & $1.78 \mathrm{e}-09$ \\
\hline 51 & $3 \mathrm{~s}^{2} 3 \mathrm{p}^{2}\left({ }_{2}^{2} \mathrm{P}\right){ }^{3} \mathrm{P} 4 \mathrm{p}^{4} \mathrm{D}_{7 / 2}^{\circ}$ & 0.92 & 202176 & 202366.62 & $1.75 \mathrm{e}-09$ & $1.78 \mathrm{e}-09$ \\
\hline 52 & $3 \mathrm{~s}^{2} 3 \mathrm{p}^{2}\left({ }_{2}^{3} \mathrm{P}\right){ }^{3} \mathrm{P} 4 \mathrm{p}^{4} \mathrm{P}_{1 / 2}^{\circ}$ & 0.92 & 203807 & 204021.26 & $1.67 \mathrm{e}-09$ & $1.69 \mathrm{e}-09$ \\
\hline 53 & $3 \mathrm{~s}^{2} 3 \mathrm{p}^{2}\left({ }_{2}^{3} \mathrm{P}\right){ }^{3} \mathrm{P} 4 \mathrm{p}^{4} \mathrm{P}_{3 / 2}^{\mathrm{o}}$ & $0.85+0.043 \mathrm{~s}^{2} 3 \mathrm{p}^{2}\left({ }_{2}^{3} \mathrm{P}\right){ }^{3} \mathrm{P} 4 \mathrm{p}^{4} \mathrm{~S}^{\circ}+0.033 \mathrm{~s}^{2} 3 \mathrm{p}^{2}\left({ }_{2}^{3} \mathrm{P}\right){ }^{3} \mathrm{P} 4 \mathrm{p}^{2} \mathrm{D}^{\circ}$ & 203902 & 204124.04 & $1.68 \mathrm{e}-09$ & $1.70 \mathrm{e}-09$ \\
\hline 54 & $3 \mathrm{~s}^{2} 3 \mathrm{p}^{2}\left({ }_{2}^{3} \mathrm{P}\right){ }^{3} \mathrm{P} 4 \mathrm{p}^{4} \mathrm{P}_{5 / 2}^{\mathrm{o} / 2}$ & $0.84+0.063 \mathrm{~s}^{2} 3 \mathrm{p}^{2}\left({ }_{2}^{3} \mathrm{P}\right){ }^{3} \mathrm{P} 4 \mathrm{p}^{2} \mathrm{D}^{\circ}{ }^{2}$ & 204320 & 204540.95 & $1.72 \mathrm{e}-09$ & $1.74 \mathrm{e}-09$ \\
\hline 55 & $3 \mathrm{~s}^{2} 3 \mathrm{p}^{2}\left({ }_{2}^{2} \mathrm{P}\right){ }^{3} \mathrm{P} 4 \mathrm{p}^{2} \mathrm{D}_{3 / 2}^{\circ}$ & $0.76+0.143 \mathrm{~s}^{2} 3 \mathrm{p}^{2}\left({ }_{2}^{1} \mathrm{D}\right){ }^{1} \mathrm{D} 4 \mathrm{p}^{2} \mathrm{D}^{\circ}+0.033 \mathrm{~s}^{2} 3 \mathrm{p}^{2}\left({ }_{2}^{3} \mathrm{P}\right){ }^{3} \mathrm{P} 4 \mathrm{p}^{4} \mathrm{P}^{\circ}$ & 204903 & 205036.99 & $2.59 \mathrm{e}-09$ & $2.59 \mathrm{e}-09$ \\
\hline 56 & $3 \mathrm{~s}^{2} 3 \mathrm{p}^{2}\left({ }_{2}^{3} \mathrm{P}\right){ }^{3} \mathrm{P} 4 \mathrm{p}^{4} \mathrm{~S}_{3 / 2}^{\circ}$ & $0.87+0.053 \mathrm{~s}^{2} 3 \mathrm{p}^{2}\left({ }_{2}^{3} \mathrm{P}\right){ }^{3} \mathrm{P} 4 \mathrm{p}^{4} \mathrm{P}^{\circ}$ & 205705 & 205938.06 & $1.49 \mathrm{e}-09$ & $1.51 \mathrm{e}-09$ \\
\hline 57 & $3 \mathrm{~s}^{2} 3 \mathrm{p}^{2}\left({ }_{2}^{2} \mathrm{P}\right){ }^{3} \mathrm{P} 4 \mathrm{p}^{2} \mathrm{D}_{5 / 2}^{\circ}$ & $0.72+0.123 \mathrm{~s}^{2} 3 \mathrm{p}^{2}\left({ }_{2}^{1} \mathrm{D}\right){ }^{1} \mathrm{D} 4 \mathrm{p}^{2} \mathrm{D}^{\circ}+0.083 \mathrm{~s}^{2} 3 \mathrm{p}^{2}\left({ }_{2}^{3} \mathrm{P}\right){ }^{3} \mathrm{P} 4 \mathrm{p}^{4} \mathrm{P}^{\circ}$ & 205802 & $205946.9 ?$ & $2.52 \mathrm{e}-09$ & $2.53 e-09$ \\
\hline 58 & $3 \mathrm{~s}^{2} 3 \mathrm{p}^{2}\left({ }_{0}^{1} \mathrm{~S}\right){ }^{1} \mathrm{~S} 3 \mathrm{~d}^{2} \mathrm{D}_{5 / 2}^{\mathrm{S} / 2}$ & $0.40+0.283 \mathrm{~s}^{2} 3 \mathrm{p}^{2}\left({ }_{2}^{3} \mathrm{P}\right){ }^{3} \mathrm{P} 3 \mathrm{~d}^{2} \mathrm{D}+0.093 \mathrm{~s}^{2} 3 \mathrm{p}^{2}\left({ }_{2}^{1} \mathrm{D}\right){ }^{1} \mathrm{D} 3 \mathrm{~d}^{2} \mathrm{D}$ & 206010 & & $4.67 \mathrm{e}-11$ & $4.65 \mathrm{e}-11$ \\
\hline 59 & $3 \mathrm{~s}^{2} 3 \mathrm{p}^{2}\left({ }_{0}^{1} \mathrm{~S}\right){ }^{1} \mathrm{~S} 3 \mathrm{~d}^{2} \mathrm{D}_{3 / 2}$ & $0.42+0.293 \mathrm{~s}^{2} 3 \mathrm{p}^{2}\left({ }_{2}^{3} \mathrm{P}\right){ }^{3} \mathrm{P} 3 \mathrm{~d}^{2} \mathrm{D}+0.083 \mathrm{~s}^{2} 3 \mathrm{p}^{2}\left({ }_{2}^{1} \mathrm{D}\right){ }^{1} \mathrm{D} 3 \mathrm{~d}^{2} \mathrm{D}$ & 206251 & & $4.73 e-11$ & $4.71 \mathrm{e}-11$ \\
\hline 60 & $3 \mathrm{~s}^{2} 3 \mathrm{p}^{2}\left({ }_{2}^{3} \mathrm{P}\right){ }^{3} \mathrm{P} 4 \mathrm{p}^{2} \mathrm{P}_{1 / 2}^{\circ}$ & $0.80+0.103 \mathrm{~s}^{2} 3 \mathrm{p}^{2}\left({ }_{2}^{1} \mathrm{D}\right){ }^{1} \mathrm{D} 4 \mathrm{p}^{2} \mathrm{P}^{\mathrm{o}}$ & 208929 & $209042.8 ?$ & $2.18 \mathrm{e}-09$ & $2.19 \mathrm{e}-09$ \\
\hline 61 & $3 \mathrm{~s}^{2} 3 \mathrm{p}^{2}\left({ }_{2}^{3} \mathrm{P}\right){ }^{3} \mathrm{P} 4 \mathrm{p}^{2} \mathrm{P}_{3 / 2}^{\mathrm{o}}$ & $0.78+0.123 \mathrm{~s}^{2} 3 \mathrm{p}^{2}\left({ }_{2}^{1} \mathrm{D}\right){ }^{1} \mathrm{D} 4 \mathrm{p}^{2} \mathrm{P}^{\circ}$ & 209054 & $209182.8 ?$ & $2.18 \mathrm{e}-09$ & $2.19 \mathrm{e}-09$ \\
\hline 62 & $3 s^{2} 3 p^{2}\left({ }_{0}^{1} S\right){ }^{1} S 4 s^{2} S_{1 / 2}^{3 / 2}$ & $0.65+0.173 \mathrm{~s}^{2} 3 \mathrm{p}^{2}\left({ }_{2}^{1} \mathrm{D}\right){ }^{1} \mathrm{D} 3 \mathrm{~d}^{2} \mathrm{~S}+0.093 \mathrm{~s}^{2} \mathrm{~S} 3 \mathrm{p}^{4}\left({ }_{0}^{1} \mathrm{~S}\right){ }^{2} \mathrm{~S}$ & 210651 & & $1.81 \mathrm{e}-09$ & $1.75 \mathrm{e}-09$ \\
\hline 63 & $3 s^{2} S 3 p^{3}\left({ }_{3}^{4} S\right){ }^{5} S 3 d^{6} D_{3 / 2}^{\circ}$ & 0.97 & 212608 & & $3.79 \mathrm{e}-06$ & $3.63 e-06$ \\
\hline 64 & $3 s^{2} S 3 p^{3}\left({ }_{3}^{4} S\right){ }^{5} S 3 d^{6} D_{5 / 2}^{\circ / 2}$ & 0.97 & 212608 & & $5.48 \mathrm{e}-06$ & $5.56 \mathrm{e}-06$ \\
\hline 65 & $3 \mathrm{~s}^{2} \mathrm{~S} 3 \mathrm{p}^{3}\left({ }_{3}^{4} \mathrm{~S}\right){ }^{5} \mathrm{~S} 3 \mathrm{~d}^{6} \mathrm{D}_{1 / 2}^{\circ / 2}$ & 0.97 & 212616 & & $3.24 \mathrm{e}-06$ & $3.06 \mathrm{e}-06$ \\
\hline 66 & $3 \mathrm{~s}^{2} \mathrm{~S} 3 \mathrm{p}^{3}\left({ }_{3}^{4} \mathrm{~S}\right){ }^{5} \mathrm{~S} 3 \mathrm{~d}^{6} \mathrm{D}_{7}^{\circ}$ & 0.97 & 212620 & & $1.07 \mathrm{e}-05$ & $1.14 \mathrm{e}-05$ \\
\hline 67 & $3 \mathrm{~s}^{2} \mathrm{~S} 3 \mathrm{p}^{3}\left({ }_{3}^{4} \mathrm{~S}\right){ }^{5} \mathrm{~S} 3 \mathrm{~d}^{6} \mathrm{D}_{9}^{\circ}$ & 0.97 & 212654 & & $3.05 \mathrm{e}-05$ & $2.52 \mathrm{e}-05$ \\
\hline 68 & $3 \mathrm{~s}^{2} 3 \mathrm{p}^{2}\left({ }_{2}^{1} \mathrm{D}\right){ }^{1} \mathrm{D} 4 \mathrm{p}^{2} \mathrm{~F}_{5 / 2}^{\circ / 2}$ & $0.91+0.023 \mathrm{~s}^{2} \mathrm{~S} 3 \mathrm{p}^{2}\left({ }_{2}^{3} \mathrm{P}\right){ }^{2} \mathrm{P} 3 \mathrm{~d}^{1} \mathrm{D} 4 \mathrm{p}^{2} \mathrm{~F}^{\circ}$ & 216534 & $216524.6 ?$ & $1.96 \mathrm{e}-09$ & $1.98 \mathrm{e}-09$ \\
\hline 69 & $3 \mathrm{~s}^{2} 3 \mathrm{p}^{2}\left({ }_{2}^{1} \mathrm{D}\right){ }^{1} \mathrm{D} 4 \mathrm{p}^{2} \mathrm{~F}_{7 / 2}^{\mathrm{o} / 2}$ & $0.92+0.023 \mathrm{~s}^{2} \mathrm{~S} 3 \mathrm{p}^{2}\left({ }_{2}^{3} \mathrm{P}\right){ }^{2} \mathrm{P} 3 \mathrm{~d}^{1} \mathrm{D} 4 \mathrm{p}^{2} \mathrm{~F}^{\circ}$ & 216726 & $216710.4 ?$ & $1.95 \mathrm{e}-09$ & $1.96 \mathrm{e}-09$ \\
\hline 70 & $3 \mathrm{~s}^{2} 3 \mathrm{p}^{2}\left({ }_{2}^{1} \mathrm{D}\right){ }^{1} \mathrm{D} 4 \mathrm{p}^{2} \mathrm{D}_{5 / 2}^{\circ}$ & $0.77+0.143 \mathrm{~s}^{2} 3 \mathrm{p}^{2}\left({ }_{2}^{3} \mathrm{P}\right){ }^{3} \mathrm{P} 4 \mathrm{p}^{2} \mathrm{D}^{\circ}$ & 217852 & $217850.2 ?$ & $2.04 \mathrm{e}-09$ & $2.06 \mathrm{e}-09$ \\
\hline 71 & $3 \mathrm{~s}^{2} 3 \mathrm{p}^{2}\left({ }_{2}^{1} \mathrm{D}\right){ }^{1} \mathrm{D} 4 \mathrm{p}^{2} \mathrm{D}_{3 / 2}^{\circ}$ & $0.77+0.143 \mathrm{~s}^{2} 3 \mathrm{p}^{2}\left({ }_{2}^{3} \mathrm{P}\right){ }^{3} \mathrm{P} 4 \mathrm{p}^{2} \mathrm{D}^{\circ}$ & 217923 & $217913.1 ?$ & $2.04 \mathrm{e}-09$ & $2.06 \mathrm{e}-09$ \\
\hline 72 & $3 \mathrm{~s}^{2} 3 \mathrm{p}^{2}\left({ }_{2}^{1} \mathrm{D}\right){ }^{1} \mathrm{D} 4 \mathrm{p}^{2} \mathrm{P}_{1 / 2}^{\mathrm{o} / 2}$ & $0.79+0.103 \mathrm{~s}^{2} 3 \mathrm{p}^{2}\left({ }_{2}^{3} \mathrm{P}\right){ }^{3} \mathrm{P} 4 \mathrm{p}^{2} \mathrm{P}^{\circ}$ & 221952 & $221862.9 ?$ & $1.86 \mathrm{e}-09$ & $1.88 \mathrm{e}-09$ \\
\hline 73 & $3 \mathrm{~s}^{2} 3 \mathrm{p}^{2}\left({ }_{2}^{1} \mathrm{D}\right){ }^{1} \mathrm{D} 4 \mathrm{p}^{2} \mathrm{P}_{3 / 2}^{\mathrm{o}}$ & $0.75+0.123 \mathrm{~s}^{2} 3 \mathrm{p}^{2}\left({ }_{2}^{3} \mathrm{P}\right){ }^{3} \mathrm{P} 4 \mathrm{p}^{2} \mathrm{P}^{\circ}$ & 222175 & $222100.7 ?$ & $1.93 \mathrm{e}-09$ & $1.96 \mathrm{e}-09$ \\
\hline 74 & $3 \mathrm{p}^{52} \mathrm{P}_{3 / 2}^{\circ}$ & $0.40+0.353 \mathrm{~s}^{2} \mathrm{~S} 3 \mathrm{p}^{3}\left({ }_{3}^{2} \mathrm{D}\right){ }^{3} \mathrm{D} 3 \mathrm{~d}^{2} \mathrm{P}^{\circ}+0.063 \mathrm{~s}^{2} \mathrm{~S} 3 \mathrm{p}^{3}\left({ }_{1}^{2} \mathrm{P}\right){ }^{3} \mathrm{P} 3 \mathrm{~d}^{2} \mathrm{P}^{\circ}$ & 230878 & 292470[] & $1.71 \mathrm{e}-09$ & $1.66 \mathrm{e}-09$ \\
\hline 75 & $3 \mathrm{p}^{5}{ }^{2} \mathrm{P}_{1 / 2}^{3 / 2}$ & $0.39+0.353 \mathrm{~s}^{2} \mathrm{~S} 3 \mathrm{p}^{3}\left({ }_{3}^{2} \mathrm{D}\right){ }^{3} \mathrm{D} 3 \mathrm{~d}^{2} \mathrm{P}^{\circ}+0.063 \mathrm{~s}^{2} \mathrm{~S} 3 \mathrm{p}^{3}\left({ }_{1}^{2} \mathrm{P}\right){ }^{3} \mathrm{P} 3 \mathrm{~d}^{2} \mathrm{P}^{\circ}$ & 231746 & 293540[] & $1.86 \mathrm{e}-09$ & $1.81 \mathrm{e}-09$ \\
\hline 76 & $3 \mathrm{~s}^{2} \mathrm{~S} 3 \mathrm{p}^{3}\left({ }_{3}^{4} \mathrm{~S}\right){ }^{5} \mathrm{~S} 3 \mathrm{~d}^{4} \mathrm{D}_{7}^{\circ}$ & $0.45+0.403 \mathrm{~s}^{2} \mathrm{~S} 3 \mathrm{p}^{3}\left({ }_{3}^{2} \mathrm{D}\right){ }^{3} \mathrm{D} 3 \mathrm{~d}^{4} \mathrm{D}^{\circ}+0.043 \mathrm{~s}^{2} 3 \mathrm{p}^{2} \mathrm{P} 3 \mathrm{~d}^{2}\left({ }_{2}^{3} \mathrm{~F}\right){ }^{4} \mathrm{D}^{\circ}$ & 233460 & & $8.10 \mathrm{e}-09$ & $7.93 e-09$ \\
\hline 77 & $3 s^{2} S 3 p^{3}\left({ }_{3}^{4} S\right){ }^{5} S 3 d^{4} D_{5 / 2}^{\circ}$ & $0.45+0.403 \mathrm{~s}^{2} \mathrm{~S} 3 \mathrm{p}^{3}\left({ }_{3}^{2} \mathrm{D}\right){ }^{3} \mathrm{D} 3 \mathrm{~d}^{4} \mathrm{D}^{\circ}+0.043 \mathrm{~s}^{2} 3 \mathrm{p}^{2} \mathrm{P} 3 \mathrm{~d}^{2}\left({ }_{2}^{3} \mathrm{~F}\right){ }^{4} \mathrm{D}^{\circ}$ & 233478 & & $7.76 \mathrm{e}-09$ & $7.61 \mathrm{e}-09$ \\
\hline 78 & $3 \mathrm{~s}^{2} \mathrm{~S} 3 \mathrm{p}^{3}\left({ }_{3}^{4} \mathrm{~S}\right){ }^{5} \mathrm{~S} 3 \mathrm{~d}^{4} \mathrm{D}_{3 / 2}^{\circ}$ & $0.45+0.403 \mathrm{~s}^{2} \mathrm{~S} 3 \mathrm{p}^{3}\left({ }_{3}^{2} \mathrm{D}\right){ }^{3} \mathrm{D} 3 \mathrm{~d}^{4} \mathrm{D}^{\circ}+0.043 \mathrm{~s}^{2} 3 \mathrm{p}^{2} \mathrm{P} 3 \mathrm{~d}^{2}\left({ }_{2}^{3} \mathrm{~F}\right){ }^{4} \mathrm{D}^{\circ}$ & 233522 & & $7.57 \mathrm{e}-09$ & $7.45 \mathrm{e}-09$ \\
\hline 79 & $3 s^{2} S 3 p^{3}\left({ }_{3}^{4} S\right){ }^{5} S 3 d^{4} D_{1 / 2}^{\circ / 2}$ & $0.45+0.403 \mathrm{~s}^{2} \mathrm{~S} 3 \mathrm{p}^{3}\left({ }_{3}^{2} \mathrm{D}\right){ }^{3} \mathrm{D} 3 \mathrm{~d}^{4} \mathrm{D}^{\circ}+0.043 \mathrm{~s}^{2} 3 \mathrm{p}^{2} \mathrm{P} 3 \mathrm{~d}^{2}\left({ }_{2}^{3} \mathrm{~F}\right){ }^{4} \mathrm{D}^{\circ}$ & 233578 & & $7.45 \mathrm{e}-09$ & $7.35 \mathrm{e}-09$ \\
\hline 80 & $3 s^{2} 3 p^{2}\left({ }_{2}^{3} \mathrm{P}\right){ }^{3} \mathrm{P} 4 d^{4} \mathrm{~F}_{3 / 2}^{1 / 2}$ & 0.91 & 239418 & $239506.3 ?$ & $1.49 \mathrm{e}-09$ & $1.58 \mathrm{e}-09$ \\
\hline 81 & $3 s^{2} 3 p^{2}\left({ }_{2}^{3} \mathrm{P}\right){ }^{3} \mathrm{P} 4 \mathrm{~d}^{4} \mathrm{~F}_{5 / 2}$ & $0.90+0.033 \mathrm{~s}^{2} 3 \mathrm{p}^{2}\left({ }_{2}^{3} \mathrm{P}\right){ }^{3} \mathrm{P} 4 \mathrm{~d}^{4} \mathrm{D}$ & 239640 & $239729.9 ?$ & $1.49 \mathrm{e}-09$ & $1.58 \mathrm{e}-09$ \\
\hline 82 & $3 \mathrm{~s}^{2} 3 \mathrm{p}^{2}\left({ }_{2}^{3} \mathrm{P}\right){ }^{3} \mathrm{P} 4 \mathrm{~d}^{4} \mathrm{~F}_{7 / 2}$ & $0.89+0.033 \mathrm{~s}^{2} 3 \mathrm{p}^{2}\left({ }_{2}^{3} \mathrm{P}\right){ }^{3} \mathrm{P} 4 \mathrm{~d}^{4} \mathrm{D}$ & 239991 & $240075.2 ?$ & $1.50 \mathrm{e}-09$ & $1.58 \mathrm{e}-09$ \\
\hline 83 & $3 \mathrm{~s}^{2} 3 \mathrm{p}^{2}\left({ }_{2}^{2} \mathrm{P}\right){ }^{3} \mathrm{P} 4 \mathrm{~d}^{4} \mathrm{~F}_{9 / 2}$ & 0.93 & 240490 & $240568.4 ?$ & $1.51 \mathrm{e}-09$ & $1.60 \mathrm{e}-09$ \\
\hline 84 & $3 \mathrm{~s}^{2} 3 \mathrm{p}^{2}\left({ }_{2}^{3} \mathrm{P}\right){ }^{3} \mathrm{P} 4 \mathrm{~d}^{4} \mathrm{D}_{1 / 2}$ & $0.88+0.043 \mathrm{~s}^{2} 3 \mathrm{p}^{2}\left({ }_{2}^{3} \mathrm{P}\right){ }^{3} \mathrm{P} 4 \mathrm{~d}^{2} \mathrm{P}$ & 241426 & $241559.4 ?$ & $1.33 \mathrm{e}-09$ & $1.38 \mathrm{e}-09$ \\
\hline 85 & $3 \mathrm{~s}^{2} 3 \mathrm{p}^{2}\left({ }_{2}^{3} \mathrm{P}\right){ }^{3} \mathrm{P} 4 \mathrm{~d}^{4} \mathrm{D}_{3 / 2}$ & $0.80+0.063 \mathrm{~s}^{2} 3 \mathrm{p}^{2}\left({ }_{2}^{3} \mathrm{P}\right){ }^{3} \mathrm{P} 4 \mathrm{~d}^{2} \mathrm{P}+0.063 \mathrm{~s}^{2} 3 \mathrm{p}^{2}\left({ }_{2}^{3} \mathrm{P}\right){ }^{3} \mathrm{P} 4 \mathrm{~d}^{4} \mathrm{P}$ & 241445 & $241572.4 ?$ & $1.17 \mathrm{e}-09$ & $1.21 \mathrm{e}-09$ \\
\hline 86 & $3 \mathrm{~s}^{2} 3 \mathrm{p}^{2}\left({ }_{2}^{3} \mathrm{P}\right){ }^{3} \mathrm{P} 4 \mathrm{~d}^{4} \mathrm{D}_{5 / 2}$ & $0.76+0.143 \mathrm{~s}^{2} 3 \mathrm{p}^{2}\left({ }_{2}^{3} \mathrm{P}\right){ }^{3} \mathrm{P} 4 \mathrm{~d}^{4} \mathrm{P}+0.033 \mathrm{~s}^{2} 3 \mathrm{p}^{2}\left({ }_{2}^{3} \mathrm{P}\right){ }^{3} \mathrm{P} 4 \mathrm{~d}^{4} \mathrm{~F}$ & 241557 & $241685.1 ?$ & $1.13 \mathrm{e}-09$ & $1.16 \mathrm{e}-09$ \\
\hline 87 & $3 s^{2} 3 p^{2}\left({ }_{2}^{3} \mathrm{P}\right){ }^{3} \mathrm{P} 4 \mathrm{~d}^{4} \mathrm{D}_{7 / 2}$ & $0.88+0.033 \mathrm{~s}^{2} 3 \mathrm{p}^{2}\left({ }_{2}^{3} \mathrm{P}\right){ }^{3} \mathrm{P} 4 \mathrm{~d}^{4} \mathrm{~F}$ & 241904 & $242046.2 ?$ & $1.50 \mathrm{e}-09$ & $1.57 \mathrm{e}-09$ \\
\hline
\end{tabular}


P. Rynkun et al.: Theoretical investigation of energy levels and transition data for S II, Cl III, Ar IV

Table A.3. Wave function composition (up to three $L S$ components with a contribution $>0.02$ of the total wave function) in $L S$-coupling and energy levels $\left(\right.$ in $\mathrm{cm}^{-1}$ ) for Ar IV.

\begin{tabular}{|c|c|c|c|c|c|c|}
\hline No. & State & $L S$-composition & $E_{\mathrm{RCI}(\mathrm{CV})}$ & $E_{\mathrm{NIST}}$ & $\tau_{1}$ & $\tau_{\mathrm{v}}$ \\
\hline 1 & $3 s^{2} 3 p^{3}\left({ }_{3}^{4} S\right){ }^{4} S_{3 / 2}^{\circ}$ & $0.95+0.033 \mathrm{~s}^{2} \mathrm{~S} 3 \mathrm{p}^{3}\left({ }_{3}^{2} \mathrm{D}\right){ }^{3} \mathrm{D} 3 \mathrm{~d}^{4} \mathrm{~S}^{\circ}$ & 0 & 0 & & \\
\hline 2 & $3 s^{2} 3 p^{3}\left({ }_{3}^{2} \mathrm{D}\right){ }^{2} \mathrm{D}_{3 / 2}^{\mathrm{o} / 2}$ & 0.93 & 21309 & 21090.6 & $4.27 \mathrm{e}+01$ & $4.28 \mathrm{e}+01$ \\
\hline 3 & $3 \mathrm{~s}^{2} 3 \mathrm{p}^{3}\left({ }_{3}^{2} \mathrm{D}\right){ }^{2} \mathrm{D}_{5 / 2}^{\circ}$ & 0.94 & 21433 & 21219.8 & $5.13 \mathrm{e}+02$ & $5.30 \mathrm{e}+02$ \\
\hline 4 & $3 \mathrm{~s}^{2} 3 \mathrm{p}^{3}\left({ }_{1}^{2} \mathrm{P}\right){ }^{2} \mathrm{P}_{1 / 2}^{\mathrm{o} / 2}$ & 0.93 & 35210 & 34854.6 & $6.08 \mathrm{e}-01$ & $6.09 \mathrm{e}-01$ \\
\hline 5 & $3 \mathrm{~s}^{2} 3 \mathrm{p}^{3}\left({ }_{1}^{2} \mathrm{P}\right){ }^{2} \mathrm{P}_{3 / 2}^{\circ}$ & 0.92 & 35372 & 35032.4 & $2.73 e-01$ & $2.73 e-01$ \\
\hline 6 & $3 \mathrm{~s}^{2} \mathrm{~S} 3 \mathrm{p}^{4}\left({ }_{2}^{3} \mathrm{P}\right){ }^{4} \mathrm{P}_{5 / 2}$ & $0.85+0.123 \mathrm{~s}^{2} 3 \mathrm{p}^{2}\left({ }_{2}^{3} \mathrm{P}\right){ }^{3} \mathrm{P} 3 \mathrm{~d}^{4} \mathrm{P}$ & 118023 & 117563.4 & $4.09 \mathrm{e}-09$ & $3.80 \mathrm{e}-09$ \\
\hline 7 & $3 \mathrm{~s}^{2} \mathrm{~S} 3 \mathrm{p}^{4}\left({ }_{2}^{3} \mathrm{P}\right){ }^{4} \mathrm{P}_{3 / 2}$ & $0.84+0.123 \mathrm{~s}^{2} 3 \mathrm{p}^{2}\left({ }_{2}^{3} \mathrm{P}\right){ }^{3} \mathrm{P} 3 \mathrm{~d}^{4} \mathrm{P}$ & 118971 & 118515.7 & $4.02 \mathrm{e}-09$ & $3.75 \mathrm{e}-09$ \\
\hline 8 & $3 \mathrm{~s}^{2} \mathrm{~S} 3 \mathrm{p}^{4}\left({ }_{2}^{3} \mathrm{P}\right){ }^{4} \mathrm{P}_{1 / 2}$ & $0.84+0.123 \mathrm{~s}^{2} 3 \mathrm{p}^{2}\left({ }_{2}^{3} \mathrm{P}\right){ }^{3} \mathrm{P} 3 \mathrm{~d}^{4} \mathrm{P}$ & 119508 & 119043.1 & $3.98 \mathrm{e}-09$ & $3.71 \mathrm{e}-09$ \\
\hline 9 & $3 \mathrm{~s}^{2} \mathrm{~S} 3 \mathrm{p}^{4}\left({ }_{2}^{1} \mathrm{D}\right){ }^{2} \mathrm{D}_{3 / 2}$ & $0.70+0.213 \mathrm{~s}^{2} 3 \mathrm{p}^{2}\left({ }_{2}^{1} \mathrm{D}\right){ }^{1} \mathrm{D} 3 \mathrm{~d}^{2} \mathrm{D}+0.033 \mathrm{~s}^{2} 3 \mathrm{p}^{2}\left({ }_{0}^{1} \mathrm{~S}\right){ }^{1} \mathrm{~S} 3 \mathrm{~d}^{2} \mathrm{D}$ & 146525 & 145921.2 & $2.17 \mathrm{e}-09$ & $2.02 \mathrm{e}-09$ \\
\hline 10 & $3 \mathrm{~s}^{2} \mathrm{~S} 3 \mathrm{p}^{4}\left({ }_{2}^{1} \mathrm{D}\right){ }^{2} \mathrm{D}_{5 / 2}$ & $0.70+0.213 \mathrm{~s}^{2} 3 \mathrm{p}^{2}\left({ }_{2}^{1} \mathrm{D}\right){ }^{1} \mathrm{D} 3 \mathrm{~d}^{2} \mathrm{D}+0.033 \mathrm{~s}^{2} 3 \mathrm{p}^{2}\left({ }_{0}^{1} \mathrm{~S}\right){ }^{1} \mathrm{~S} 3 \mathrm{~d}^{2} \mathrm{D}$ & 146603 & 145999.4 & $2.23 \mathrm{e}-09$ & $2.07 \mathrm{e}-09$ \\
\hline 11 & $3 \mathrm{~s}^{2} 3 \mathrm{p}^{2}\left({ }_{2}^{3} \mathrm{P}\right){ }^{3} \mathrm{P} 3 \mathrm{~d}^{2} \mathrm{P}_{3 / 2}$ & $0.55+0.323 \mathrm{~s}^{2} \mathrm{~S} 3 \mathrm{p}^{4}\left({ }_{2}^{3} \mathrm{P}\right){ }^{2} \mathrm{P}+0.073 \mathrm{~s}^{2} 3 \mathrm{p}^{2}\left({ }_{2}^{1} \mathrm{D}\right){ }^{1} \mathrm{D} 3 \mathrm{~d}^{2} \mathrm{P}$ & 166719 & 166356.4 & $1.01 \mathrm{e}-09$ & $9.54 \mathrm{e}-10$ \\
\hline 12 & $3 s^{2} 3 p^{2}\left({ }_{2}^{3} \mathrm{P}\right){ }^{3} \mathrm{P} 3 \mathrm{~d}^{2} \mathrm{P}_{1 / 2}$ & $0.55+0.323 \mathrm{~s}^{2} \mathrm{~S} 3 \mathrm{p}^{4}\left({ }_{2}^{3} \mathrm{P}\right){ }^{2} \mathrm{P}+0.063 \mathrm{~s}^{2} 3 \mathrm{p}^{2}\left({ }_{2}^{1} \mathrm{D}\right){ }^{1} \mathrm{D} 3 \mathrm{~d}^{2} \mathrm{P}$ & 167826 & 167444.6 & $9.85 \mathrm{e}-10$ & $9.27 \mathrm{e}-10$ \\
\hline 13 & $3 \mathrm{~s}^{2} \mathrm{~S} 3 \mathrm{p}^{4}\left({ }_{0}^{1} \mathrm{~S}\right)^{2} \mathrm{~S}_{1 / 2}$ & $0.72+0.233 \mathrm{~s}^{2} 3 \mathrm{p}^{2}\left({ }_{2}^{1} \mathrm{D}\right){ }^{1} \mathrm{D} 3 \mathrm{~d}^{2} \mathrm{~S}$ & 178782 & 177832.5 & $6.65 \mathrm{e}-10$ & $6.30 \mathrm{e}-10$ \\
\hline 14 & $3 \mathrm{~s}^{2} 3 \mathrm{p}^{2}\left({ }_{2}^{3} \mathrm{P}\right){ }^{3} \mathrm{P} 3 \mathrm{~d}^{4} \mathrm{~F}_{3 / 2}$ & 0.95 & 180820 & 180682.5 & $4.56 \mathrm{e}-07$ & $4.37 \mathrm{e}-07$ \\
\hline 15 & $3 s^{2} 3 p^{2}\left({ }_{2}^{3} \mathrm{P}\right){ }^{3} \mathrm{P} 3 \mathrm{~d}^{4} \mathrm{~F}_{5 / 2}$ & 0.94 & 181169 & 181031.8 & $6.05 \mathrm{e}-07$ & $6.00 \mathrm{e}-07$ \\
\hline 16 & $3 s^{2} 3 p^{2}\left({ }_{2}^{3} \mathrm{P}\right){ }^{3} \mathrm{P} 3 \mathrm{~d}^{4} \mathrm{~F}_{7 / 2}$ & 0.94 & 181686 & 181533.3 & $1.07 \mathrm{e}-06$ & $1.11 \mathrm{e}-06$ \\
\hline 17 & $3 s^{2} 3 p^{2}\left({ }_{2}^{3} \mathrm{P}\right){ }^{3} \mathrm{P} 3 \mathrm{~d}^{4} \mathrm{~F}_{9 / 2}$ & 0.95 & 182360 & 182195.6 & $1.40 \mathrm{e}-01$ & $1.36 \mathrm{e}-01$ \\
\hline 18 & $3 \mathrm{~s}^{2} 3 \mathrm{p}^{2}\left({ }_{2}^{1} \mathrm{D}\right){ }^{1} \mathrm{D} 3 \mathrm{~d}^{2} \mathrm{~F}_{5 / 2}$ & $0.38+0.303 \mathrm{~s}^{2} 3 \mathrm{p}^{2}\left({ }_{2}^{3} \mathrm{P}\right){ }^{3} \mathrm{P} 3 \mathrm{~d}^{4} \mathrm{D}+0.263 \mathrm{~s}^{2} 3 \mathrm{p}^{2}\left({ }_{2}^{3} \mathrm{P}\right){ }^{3} \mathrm{P} 3 \mathrm{~d}^{2} \mathrm{~F}$ & 187234 & & $1.91 \mathrm{e}-07$ & $1.92 \mathrm{e}-07$ \\
\hline 19 & $3 s^{2} 3 p^{2}\left({ }_{2}^{3} \mathrm{P}\right){ }^{3} \mathrm{P} 3 \mathrm{~d}^{4} \mathrm{D}_{1 / 2}$ & 0.94 & 187477 & 187290.4 & $1.54 \mathrm{e}-07$ & $1.48 \mathrm{e}-07$ \\
\hline 20 & $3 \mathrm{~s}^{2} 3 \mathrm{p}^{2}\left({ }_{2}^{3} \mathrm{P}\right){ }^{3} \mathrm{P} 3 \mathrm{~d}^{4} \mathrm{D}_{7 / 2}$ & $0.59+0.213 \mathrm{~s}^{2} 3 \mathrm{p}^{2}\left({ }_{2}^{1} \mathrm{D}\right){ }^{1} \mathrm{D} 3 \mathrm{~d}^{2} \mathrm{~F}+0.143 \mathrm{~s}^{2} 3 \mathrm{p}^{2}\left({ }_{2}^{3} \mathrm{P}\right){ }^{3} \mathrm{P} 3 \mathrm{~d}^{2} \mathrm{~F}$ & 187506 & 188824.5 & $5.55 \mathrm{e}-05$ & $4.31 \mathrm{e}-05$ \\
\hline 21 & $3 \mathrm{~s}^{2} 3 \mathrm{p}^{2}\left({ }_{2}^{3} \mathrm{P}\right){ }^{3} \mathrm{P} 3 \mathrm{~d}^{4} \mathrm{D}_{3 / 2}$ & 0.94 & 187575 & 187397.5 & $1.19 \mathrm{e}-07$ & $1.17 \mathrm{e}-07$ \\
\hline 22 & $3 s^{2} 3 p^{2}\left({ }_{2}^{3} \mathrm{P}\right){ }^{3} \mathrm{P} 3 \mathrm{~d}^{4} \mathrm{D}_{5 / 2}$ & $0.64+0.183 \mathrm{~s}^{2} 3 \mathrm{p}^{2}\left({ }_{2}^{1} \mathrm{D}\right){ }^{1} \mathrm{D} 3 \mathrm{~d}^{2} \mathrm{~F}+0.133 \mathrm{~s}^{2} 3 \mathrm{p}^{2}\left({ }_{2}^{3} \mathrm{P}\right){ }^{3} \mathrm{P} 3 \mathrm{~d}^{2} \mathrm{~F}$ & 188019 & 187821.3 & $1.18 \mathrm{e}-07$ & $1.18 \mathrm{e}-07$ \\
\hline 23 & $3 \mathrm{~s}^{2} 3 \mathrm{p}^{2}\left({ }_{2}^{1} \mathrm{D}\right){ }^{1} \mathrm{D} 3 \mathrm{~d}^{2} \mathrm{~F}_{7 / 2}$ & $0.35+0.353 \mathrm{~s}^{2} 3 \mathrm{p}^{2}\left({ }_{2}^{3} \mathrm{P}\right){ }^{3} \mathrm{P} 3 \mathrm{~d}^{4} \mathrm{D}+0.243 \mathrm{~s}^{2} 3 \mathrm{p}^{2}\left({ }_{2}^{3} \mathrm{P}\right){ }^{3} \mathrm{P} 3 \mathrm{~d}^{2} \mathrm{~F}$ & 189057 & & $7.89 \mathrm{e}-07$ & $7.76 \mathrm{e}-07$ \\
\hline 24 & $3 \mathrm{~s}^{2} 3 \mathrm{p}^{2}\left({ }_{2}^{1} \mathrm{D}\right){ }^{1} \mathrm{D} 3 \mathrm{~d}^{2} \mathrm{G}_{7 / 2}$ & 0.94 & 208246 & 207760.7 & $1.93 \mathrm{e}-07$ & $1.94 \mathrm{e}-07$ \\
\hline 25 & $3 \mathrm{~s}^{2} 3 \mathrm{p}^{2}\left({ }_{2}^{1} \mathrm{D}\right){ }^{1} \mathrm{D} 3 \mathrm{~d}^{2} \mathrm{G}_{9 / 2}$ & 0.94 & 208374 & 207859.3 & $1.26 \mathrm{e}-01$ & $1.24 \mathrm{e}-01$ \\
\hline 26 & $3 s^{2} 3 p^{2}\left({ }_{2}^{2} \mathrm{P}\right){ }^{3} \mathrm{P} 3 \mathrm{~d}^{4} \mathrm{P}_{5 / 2}$ & $0.81+0.113 \mathrm{~s}^{2} \mathrm{~S} 3 \mathrm{p}^{4}\left({ }_{2}^{3} \mathrm{P}\right){ }^{4} \mathrm{P}$ & 221139 & 220786.8 & $3.07 \mathrm{e}-11$ & $3.05 \mathrm{e}-11$ \\
\hline 27 & $3 s^{2} 3 p^{2}\left({ }_{2}^{3} \mathrm{P}\right){ }^{3} \mathrm{P} 3 \mathrm{~d}^{4} \mathrm{P}_{3 / 2}$ & $0.80+0.113 \mathrm{~s}^{2} \mathrm{~S} 3 \mathrm{p}^{4}\left({ }_{2}^{3} \mathrm{P}\right){ }^{4} \mathrm{P}$ & 221654 & 221300.9 & $3.05 \mathrm{e}-11$ & $3.03 e-11$ \\
\hline 28 & $3 s^{2} 3 p^{2}\left({ }_{2}^{3} \mathrm{P}\right){ }^{3} \mathrm{P} 3 \mathrm{~d}^{4} \mathrm{P}_{1 / 2}$ & $0.81+0.113 \mathrm{~s}^{2} \mathrm{~S} 3 \mathrm{p}^{4}\left({ }_{2}^{3} \mathrm{P}\right){ }^{4} \mathrm{P}$ & 222001 & 221627.4 & $3.03 \mathrm{e}-11$ & $3.01 \mathrm{e}-11$ \\
\hline 29 & $3 \mathrm{~s}^{2} \mathrm{~S} 3 \mathrm{p}^{4}\left({ }_{2}^{3} \mathrm{P}\right){ }^{2} \mathrm{P}_{3 / 2}$ & $0.44+0.363 \mathrm{~s}^{2} 3 \mathrm{p}^{2}\left({ }_{2}^{1} \mathrm{D}\right){ }^{1} \mathrm{D} 3 \mathrm{~d}^{2} \mathrm{P}+0.093 \mathrm{~s}^{2} 3 \mathrm{p}^{2}\left({ }_{2}^{3} \mathrm{P}\right){ }^{3} \mathrm{P} 3 \mathrm{~d}^{2} \mathrm{P}$ & 223805 & 222956.1 & $4.46 \mathrm{e}-11$ & $4.36 \mathrm{e}-11$ \\
\hline 30 & $3 \mathrm{~s}^{2} \mathrm{~S} 3 \mathrm{p}^{4}\left({ }_{2}^{3} \mathrm{P}\right){ }^{2} \mathrm{P}_{1 / 2}$ & $0.43+0.413 \mathrm{~s}^{2} 3 \mathrm{p}^{2}\left({ }_{2}^{1} \mathrm{D}\right){ }^{1} \mathrm{D} 3 \mathrm{~d}^{2} \mathrm{P}+0.083 \mathrm{~s}^{2} 3 \mathrm{p}^{2}\left({ }_{2}^{3} \mathrm{P}\right){ }^{3} \mathrm{P} 3 \mathrm{~d}^{2} \mathrm{P}$ & 224891 & 224019.3 & $4.41 \mathrm{e}-11$ & $4.32 \mathrm{e}-11$ \\
\hline 31 & $3 \mathrm{~s}^{2} 3 \mathrm{p}^{2}\left({ }_{0}^{1} \mathrm{~S}\right){ }^{1} \mathrm{~S} 3 \mathrm{~d}^{2} \mathrm{D}_{3 / 2}$ & $0.46+0.413 \mathrm{~s}^{2} 3 \mathrm{p}^{2}\left({ }_{2}^{3} \mathrm{P}\right){ }^{3} \mathrm{P} 3 \mathrm{~d}^{2} \mathrm{D}+0.023 \mathrm{~s}^{2} \mathrm{~S} 3 \mathrm{p}^{4}\left({ }_{2}^{1} \mathrm{D}\right){ }^{2} \mathrm{D}$ & 225349 & & $2.73 \mathrm{e}-10$ & $2.69 \mathrm{e}-10$ \\
\hline 32 & $3 \mathrm{~s}^{2} 3 \mathrm{p}^{2}\left({ }_{0}^{1} \mathrm{~S}\right){ }^{1} \mathrm{~S} 3 \mathrm{~d}^{2} \mathrm{D}_{5 / 2}$ & $0.47+0.423 \mathrm{~s}^{2} 3 \mathrm{p}^{2}\left({ }_{2}^{3} \mathrm{P}\right){ }^{3} \mathrm{P} 3 \mathrm{~d}^{2} \mathrm{D}+0.033 \mathrm{~s}^{2} \mathrm{~S} 3 \mathrm{p}^{4}\left({ }_{2}^{1} \mathrm{D}\right){ }^{2} \mathrm{D}$ & 226733 & & $2.65 \mathrm{e}-10$ & $2.61 \mathrm{e}-10$ \\
\hline 33 & $3 \mathrm{~s}^{2} 3 \mathrm{p}^{2}\left({ }_{2}^{1} \mathrm{D}\right){ }^{1} \mathrm{D} 3 \mathrm{~d}^{2} \mathrm{D}_{5 / 2}$ & $0.64+0.163 \mathrm{~s}^{2} \mathrm{~S} 3 \mathrm{p}^{4}\left({ }_{2}^{1} \mathrm{D}\right){ }^{2} \mathrm{D}+0.063 \mathrm{~s}^{2} 3 \mathrm{p}^{2}\left({ }_{0}^{1} \mathrm{~S}\right){ }^{1} \mathrm{~S}^{2} \mathrm{~d}^{2} \mathrm{D}$ & 239358 & 238674 & $3.84 \mathrm{e}-11$ & $3.80 \mathrm{e}-11$ \\
\hline 34 & $3 \mathrm{~s}^{2} 3 \mathrm{p}^{2}\left({ }_{2}^{1} \mathrm{D}\right){ }^{1} \mathrm{D} 3 \mathrm{~d}^{2} \mathrm{D}_{3 / 2}$ & $0.65+0.173 \mathrm{~s}^{2} \mathrm{~S} 3 \mathrm{p}^{4}\left({ }_{2}^{1} \mathrm{D}\right){ }^{2} \mathrm{D}+0.053 \mathrm{~s}^{2} 3 \mathrm{p}^{2}\left({ }_{2}^{3} \mathrm{P}\right){ }^{3} \mathrm{P} 3 \mathrm{~d}^{2} \mathrm{D}$ & 239738 & 239050 & $3.69 \mathrm{e}-11$ & $3.65 e-11$ \\
\hline 35 & $3 \mathrm{~s}^{2} 3 \mathrm{p}^{2}\left({ }_{2}^{1} \mathrm{D}\right){ }^{1} \mathrm{D} 3 \mathrm{~d}^{2} \mathrm{P}_{1 / 2}$ & $0.44+0.303 \mathrm{~s}^{2} 3 \mathrm{p}^{2}\left({ }_{2}^{3} \mathrm{P}\right){ }^{3} \mathrm{P} 3 \mathrm{~d}^{2} \mathrm{P}+0.153 \mathrm{~s}^{2} \mathrm{~S} 3 \mathrm{p}^{4}\left({ }_{2}^{3} \mathrm{P}\right){ }^{2} \mathrm{P}$ & 246701 & & $3.64 \mathrm{e}-11$ & $3.59 \mathrm{e}-11$ \\
\hline 36 & $3 \mathrm{~s}^{2} 3 \mathrm{p}^{2}\left({ }_{2}^{1} \mathrm{D}\right){ }^{1} \mathrm{D} 3 \mathrm{~d}^{2} \mathrm{P}_{3 / 2}$ & $0.47+0.303 \mathrm{~s}^{2} 3 \mathrm{p}^{2}\left({ }_{2}^{3} \mathrm{P}\right){ }^{3} \mathrm{P} 3 \mathrm{~d}^{2} \mathrm{P}+0.143 \mathrm{~s}^{2} \mathrm{~S} 3 \mathrm{p}^{4}\left({ }_{2}^{3} \mathrm{P}\right){ }^{2} \mathrm{P}$ & 247150 & & $3.66 \mathrm{e}-11$ & $3.61 \mathrm{e}-11$ \\
\hline 37 & $\left.3 \mathrm{~s}^{2} 3 \mathrm{p}^{2}{ }^{3}{ }^{3} \mathrm{P}\right){ }^{3} \mathrm{P} 3 \mathrm{~d}^{2} \mathrm{~F}_{5 / 2}$ & $0.53+0.373 \mathrm{~s}^{2} 3 \mathrm{p}^{2}\left({ }_{2}^{1} \mathrm{D}\right){ }^{1} \mathrm{D} 3 \mathrm{~d}^{2} \mathrm{~F}$ & 247182 & 246628 & $2.61 \mathrm{e}-11$ & $2.61 \mathrm{e}-11$ \\
\hline 38 & $3 \mathrm{~s}^{2} 3 \mathrm{p}^{2}\left({ }_{2}^{3} \mathrm{P}\right){ }^{3} \mathrm{P} 3 \mathrm{~d}^{2} \mathrm{~F}_{7 / 2}$ & $0.54+0.373 \mathrm{~s}^{2} 3 \mathrm{p}^{2}\left({ }_{2}^{1} \mathrm{D}\right){ }^{1} \mathrm{D} 3 \mathrm{~d}^{2} \mathrm{~F}$ & 247316 & 246737.6 & $2.61 \mathrm{e}-11$ & $2.61 \mathrm{e}-11$ \\
\hline 39 & $3 \mathrm{~s}^{2} 3 \mathrm{p}^{2}\left({ }_{2}^{1} \mathrm{D}\right){ }^{1} \mathrm{D} 3 \mathrm{~d}^{2} \mathrm{~S}_{1 / 2}$ & $0.48+0.273 \mathrm{~s}^{2} 3 \mathrm{p}^{2}\left({ }_{2}^{3} \mathrm{P}\right){ }^{3} \mathrm{P} 4 \mathrm{~s}{ }^{4} \mathrm{P}+0.143 \mathrm{~s}^{2} \mathrm{~S} 3 \mathrm{p}^{4}\left({ }_{0}^{1} \mathrm{~S}\right)^{2} \mathrm{~S}$ & 250005 & & $5.31 \mathrm{e}-11$ & $5.26 \mathrm{e}-11$ \\
\hline 40 & $3 s^{2} 3 p^{2}\left({ }_{2}^{2} \mathrm{P}\right){ }^{3} \mathrm{P} 4 \mathrm{~s}^{4} \mathrm{P}_{1 / 2}$ & $0.66+0.203 \mathrm{~s}^{2} 3 \mathrm{p}^{2}\left({ }_{2}^{1} \mathrm{D}\right){ }^{1} \mathrm{D} 3 \mathrm{~d}^{2} \mathrm{~S}+0.063 \mathrm{~s}^{2} \mathrm{~S} 3 \mathrm{p}^{4}\left({ }_{0}^{1} \mathrm{~S}\right){ }^{2} \mathrm{~S}$ & 250061 & 250215.2 & $1.14 \mathrm{e}-10$ & $1.13 \mathrm{e}-10$ \\
\hline 41 & $3 \mathrm{~s}^{2} 3 \mathrm{p}^{2}\left({ }_{2}^{3} \mathrm{P}\right){ }^{3} \mathrm{P} 4 \mathrm{~s}^{4} \mathrm{P}_{3 / 2}$ & 0.92 & 250721 & 250902.16 & $4.87 \mathrm{e}-10$ & $4.81 \mathrm{e}-10$ \\
\hline 42 & $3 \mathrm{~s}^{2} 3 \mathrm{p}^{2}\left({ }_{2}^{3} \mathrm{P}\right){ }^{3} \mathrm{P} 4 \mathrm{~s}^{4} \mathrm{P}_{5 / 2}$ & 0.93 & 251782 & 251967.35 & $4.85 \mathrm{e}-10$ & $4.79 \mathrm{e}-10$ \\
\hline 43 & $3 \mathrm{~s}^{2} 3 \mathrm{p}^{2}\left({ }_{2}^{3} \mathrm{P}\right){ }^{3} \mathrm{P} 4 \mathrm{~s}^{2} \mathrm{P}_{1 / 2}$ & 0.91 & 255973 & 256087.8 & $1.19 \mathrm{e}-10$ & $1.18 \mathrm{e}-10$ \\
\hline 44 & $3 s^{2} 3 p^{2}\left({ }_{3}^{3} \mathrm{P}\right){ }^{3} \mathrm{P} 4 \mathrm{~s}^{2} \mathrm{P}_{3 / 2}$ & 0.91 & 257213 & 257343.58 & $1.17 \mathrm{e}-10$ & $1.16 \mathrm{e}-10$ \\
\hline 45 & $3 \mathrm{~s}^{2} 3 \mathrm{p}^{2}\left({ }_{2}^{3} \mathrm{P}\right){ }^{3} \mathrm{P} 3 \mathrm{~d}^{2} \mathrm{D}_{5 / 2}$ & $0.43+0.353 \mathrm{~s}^{2} 3 \mathrm{p}^{2}\left({ }_{0}^{1} \mathrm{~S}\right){ }^{1} \mathrm{~S} 3 \mathrm{~d}^{2} \mathrm{D}+0.073 \mathrm{~s}^{2} 3 \mathrm{p}^{2}\left({ }_{2}^{1} \mathrm{D}\right){ }^{1} \mathrm{D} 3 \mathrm{~d}^{2} \mathrm{D}$ & 257224 & & $2.91 \mathrm{e}-11$ & $2.90 \mathrm{e}-11$ \\
\hline 46 & $3 \mathrm{~s}^{2} 3 \mathrm{p}^{2}\left({ }_{2}^{2} \mathrm{P}\right){ }^{3} \mathrm{P} 3 \mathrm{~d}^{2} \mathrm{D}_{3 / 2}$ & $0.43+0.373 \mathrm{~s}^{2} 3 \mathrm{p}^{2}\left({ }_{0}^{1} \mathrm{~S}\right){ }^{1} \mathrm{~S} 3 \mathrm{~d}^{2} \mathrm{D}+0.053 \mathrm{~s}^{2} 3 \mathrm{p}^{2}\left({ }_{2}^{1} \mathrm{D}\right){ }^{1} \mathrm{D} 3 \mathrm{~d}^{2} \mathrm{D}$ & 257667 & & $2.93 \mathrm{e}-11$ & $2.92 \mathrm{e}-11$ \\
\hline 47 & $3 \mathrm{~s}^{2} \mathrm{~S} 3 \mathrm{p}^{3}\left({ }_{3}^{4} \mathrm{~S}\right){ }^{5} \mathrm{~S} 3 \mathrm{~d}^{6} \mathrm{D}_{3 / 2}^{\circ}$ & 0.98 & 259721 & & $1.72 \mathrm{e}-06$ & $1.65 \mathrm{e}-06$ \\
\hline 48 & $3 s^{2} S 3 p^{3}\left({ }_{3}^{4} S\right){ }^{5} S 3 d^{6} D_{1 / 2}^{0 / 2}$ & 0.98 & 259730 & & $1.47 \mathrm{e}-06$ & $1.38 \mathrm{e}-06$ \\
\hline 49 & $3 s^{2} S 3 p^{3}\left({ }_{3}^{4} S\right){ }^{5} S 3 d^{6} D_{5 / 2}^{\circ}$ & 0.98 & 259731 & & $2.42 \mathrm{e}-06$ & $2.41 \mathrm{e}-06$ \\
\hline 50 & $3 s^{2} S 3 p^{3}\left({ }_{3}^{4} S\right){ }^{5} S 3 d^{6} D_{7 / 2}^{\circ}$ & 0.98 & 259755 & & $4.51 \mathrm{e}-06$ & $4.67 \mathrm{e}-06$ \\
\hline 51 & $3{ }^{2} S 3 p^{3}\left({ }_{3}^{4} S\right){ }^{5} S 3 d^{6} D_{9 / 2}^{\circ}$ & 0.98 & 259799 & & $1.12 \mathrm{e}-05$ & $9.38 \mathrm{e}-06$ \\
\hline 52 & $3 \mathrm{~s}^{2} 3 \mathrm{p}^{2}\left({ }_{2}^{1} \mathrm{D}\right){ }^{1} \mathrm{D} 4 \mathrm{~s}^{2} \mathrm{D}_{5 / 2}$ & $0.92+0.023 \mathrm{~s}^{2} \mathrm{~S} 3 \mathrm{p}^{2}\left({ }_{2}^{3} \mathrm{P}\right){ }^{2} \mathrm{P} 3 \mathrm{~d}{ }^{1} \mathrm{D} 4 \mathrm{~s}^{2} \mathrm{D}$ & 267750 & 267741.3 & $2.31 \mathrm{e}-10$ & $2.29 \mathrm{e}-10$ \\
\hline 53 & $3 \mathrm{~s}^{2} 3 \mathrm{p}^{2}\left({ }_{2}^{1} \mathrm{D}\right){ }^{1} \mathrm{D} 4 \mathrm{~s}^{2} \mathrm{D}_{3 / 2}$ & $0.92+0.023 \mathrm{~s}^{2} \mathrm{~S} 3 \mathrm{p}^{2}\left({ }_{2}^{3} \mathrm{P}\right){ }^{2} \mathrm{P} 3 \mathrm{~d}^{1} \mathrm{D} 4 \mathrm{~s}^{2} \mathrm{D}$ & 267774 & 267762 & $2.29 \mathrm{e}-10$ & $2.26 \mathrm{e}-10$ \\
\hline 54 & $3 \mathrm{p}^{5}{ }^{2} \mathrm{P}_{3 / 2}^{\circ}$ & $0.48+0.343 \mathrm{~s}^{2} \mathrm{~S} 3 \mathrm{p}^{3}\left({ }_{3}^{2} \mathrm{D}\right){ }^{3} \mathrm{D} 3 \mathrm{~d}^{2} \mathrm{P}^{\circ}+0.063 \mathrm{~s}^{2} \mathrm{~S} 3 \mathrm{p}^{3}\left({ }_{1}^{2} \mathrm{P}\right){ }^{3} \mathrm{P} 3 \mathrm{~d}^{2} \mathrm{P}^{\circ}$ & 275778 & & $1.01 \mathrm{e}-09$ & $9.51 \mathrm{e}-10$ \\
\hline 55 & $3 \mathrm{p}^{5}{ }^{2} \mathrm{P}_{1 / 2}^{\mathrm{o} / 2}$ & $0.47+0.353 \mathrm{~s}^{2} \mathrm{~S} 3 \mathrm{p}^{3}\left({ }_{3}^{2} \mathrm{D}\right){ }^{3} \mathrm{D} 3 \mathrm{~d}^{2} \mathrm{P}^{\circ}+0.063 \mathrm{~s}^{2} \mathrm{~S} 3 \mathrm{p}^{3}\left({ }_{1}^{2} \mathrm{P}\right){ }^{3} \mathrm{P} 3 \mathrm{~d}^{2} \mathrm{P}^{\circ}$ & 277251 & & $1.04 \mathrm{e}-09$ & $9.78 \mathrm{e}-10$ \\
\hline
\end{tabular}

Notes. Energy levels are given relative to the ground state. The states 57 and 58 are indicated in bold. The labels for these levels were not assigned with largest contribution to the composition. 
Table A.3. continued.

\begin{tabular}{|c|c|c|c|c|c|c|}
\hline 56 & $3 \mathrm{~s}^{2} 3 \mathrm{p}^{2}\left({ }_{2}^{3} \mathrm{P}\right){ }^{3} \mathrm{P} 4 \mathrm{p}^{2} \mathrm{~S}_{1 / 2}^{\circ}$ & 0.92 & 280888 & & $9.69 \mathrm{e}-10$ & $9.64 \mathrm{e}-10$ \\
\hline 57 & $3{ }^{2} S 3 p^{3}\left({ }_{3}^{4} S\right){ }^{5} S 3 d^{4} D_{1 / 2}^{\circ}$ & $\mathbf{0 . 2 4}+0.453 \mathrm{~s}^{2} 3 \mathrm{p}^{2}\left({ }_{2}^{3} \mathrm{P}\right){ }^{3} \mathrm{P} 4 \mathrm{p}^{4} \mathrm{D}^{\circ}+0.203 \mathrm{~s}^{2} \mathrm{~S} 3 \mathrm{p}^{3}\left({ }_{3}^{2} \mathrm{D}\right){ }^{3} \mathrm{D} 3 \mathrm{~d}^{4} \mathrm{D}^{\circ}$ & 283959 & & $1.17 \mathrm{e}-09$ & $1.17 \mathrm{e}-09$ \\
\hline 58 & $3 \mathrm{~s}^{2} \mathrm{~S} 3 \mathrm{p}^{3}\left({ }_{3}^{4} \mathrm{~S}\right){ }^{5} \mathrm{~S} 3 \mathrm{~d}^{4} \mathrm{D}_{3 / 2}^{\circ}$ & $\mathbf{0 . 2 8}+0.363 \mathrm{~s}^{2} 3 \mathrm{p}^{2}\left({ }_{2}^{3} \mathrm{P}\right){ }^{3} \mathrm{P} 4 \mathrm{p}^{4} \mathrm{D}^{\circ}+0.253 \mathrm{~s}^{2} \mathrm{~S} 3 \mathrm{p}^{3}\left({ }_{3}^{2} \mathrm{D}\right){ }^{3} \mathrm{D} 3 \mathrm{~d}^{4} \mathrm{D}^{\circ}$ & 284080 & & $1.46 \mathrm{e}-09$ & $1.47 \mathrm{e}-09$ \\
\hline 59 & $3 s^{2} S 3 p^{3}\left({ }_{3}^{4} S\right){ }^{5} S 3 d^{4} D_{5 / 2}^{0 / 2}$ & $0.33+0.313 \mathrm{~s}^{2} \mathrm{~S} 3 \mathrm{p}^{3}\left({ }_{3}^{2} \mathrm{D}\right){ }^{3} \mathrm{D} 3 \mathrm{~d}^{4} \mathrm{D}^{\circ}+0.233 \mathrm{~s}^{2} 3 \mathrm{p}^{2}\left({ }_{2}^{3} \mathrm{P}\right){ }^{3} \mathrm{P} 4 \mathrm{p}^{4} \mathrm{D}^{\circ}$ & 284232 & & $2.15 \mathrm{e}-09$ & $2.16 \mathrm{e}-09$ \\
\hline 60 & $3{ }^{2} S 3 p^{3}\left({ }_{3}^{4} S\right){ }^{5} S 3 d^{4} D_{7 / 2}^{\circ / 2}$ & $0.37+0.363 \mathrm{~s}^{2} \mathrm{~S} 3 \mathrm{p}^{3}\left({ }_{3}^{2} \mathrm{D}\right){ }^{3} \mathrm{D} 3 \mathrm{~d}^{4} \mathrm{D}^{\circ}+0.143 \mathrm{~s}^{2} 3 \mathrm{p}^{2}\left({ }_{2}^{3} \mathrm{P}\right){ }^{3} \mathrm{P} 4 \mathrm{p}^{4} \mathrm{D}^{\circ}$ & 284368 & & $3.40 \mathrm{e}-09$ & $3.39 \mathrm{e}-09$ \\
\hline 61 & $3 \mathrm{~s}^{2} 3 \mathrm{p}^{2}\left({ }_{2}^{3} \mathrm{P}\right){ }^{3} \mathrm{P} 4 \mathrm{p}^{4} \mathrm{D}_{1 / 2}^{\circ}$ & $0.47+0.253 \mathrm{~s}^{2} \mathrm{~S} 3 \mathrm{p}^{3}\left({ }_{3}^{2} \mathrm{D}\right){ }^{3} \mathrm{D} 3 \mathrm{~d}^{4} \mathrm{D}^{\circ}+0.183 \mathrm{~s}^{2} \mathrm{~S} 3 \mathrm{p}^{3}\left({ }_{3}^{4} \mathrm{~S}\right){ }^{5} \mathrm{~S} 3 \mathrm{~d}^{4} \mathrm{D}^{\circ}$ & 286149 & 285956.2 & $9.93 e-10$ & $9.90 \mathrm{e}-10$ \\
\hline 62 & $3 \mathrm{~s}^{2} 3 \mathrm{p}^{2}\left({ }_{2}^{2} \mathrm{P}\right){ }^{3} \mathrm{P} 4 \mathrm{p}^{4} \mathrm{D}_{3 / 2}^{\circ}$ & $0.56+0.203 \mathrm{~s}^{2} \mathrm{~S} 3 \mathrm{p}^{3}\left({ }_{3}^{2} \mathrm{D}\right){ }^{3} \mathrm{D} 3 \mathrm{~d}^{4} \mathrm{D}^{\circ}+0.143 \mathrm{~s}^{2} \mathrm{~S} 3 \mathrm{p}^{3}\left({ }_{3}^{4} \mathrm{~S}\right){ }^{5} \mathrm{~S} 3 \mathrm{~d}^{4} \mathrm{D}^{\circ}$ & 286336 & 286224.5 & $8.45 \mathrm{e}-10$ & $8.43 e-10$ \\
\hline 63 & $3 \mathrm{~s}^{2} 3 \mathrm{p}^{2}\left({ }_{2}^{2} \mathrm{P}\right){ }^{3} \mathrm{P} 4 \mathrm{p}^{4} \mathrm{D}_{5 / 2}^{\circ}$ & $0.68+0.143 \mathrm{~s}^{2} \mathrm{~S} 3 \mathrm{p}^{3}\left({ }_{3}^{2} \mathrm{D}\right){ }^{3} \mathrm{D} 3 \mathrm{~d}^{4} \mathrm{D}^{\circ}+0.083 \mathrm{~s}^{2} \mathrm{~S} 3 \mathrm{p}^{3}\left({ }_{3}^{4} \mathrm{~S}\right){ }^{5} \mathrm{~S} 3 \mathrm{~d}^{4} \mathrm{D}^{\circ}$ & 286778 & 86747.8 & $7.14 \mathrm{e}-10$ & $7.14 \mathrm{e}-10$ \\
\hline 64 & $3 \mathrm{~s}^{2} 3 \mathrm{p}^{2}\left({ }_{2}^{3} \mathrm{P}\right){ }^{3} \mathrm{P} 4 \mathrm{p}^{4} \mathrm{D}_{7 / 2}^{\circ}$ & $0.79+0.093 \mathrm{~s}^{2} \mathrm{~S} 3 \mathrm{p}^{3}\left({ }_{3}^{2} \mathrm{D}\right){ }^{3} \mathrm{D} 3 \mathrm{~d}^{4} \mathrm{D}^{\circ}+0.043 \mathrm{~s}^{2} \mathrm{~S} 3 \mathrm{p}^{3}\left({ }_{3}^{4} \mathrm{~S}\right){ }^{5} \mathrm{~S} 3 \mathrm{~d}^{4} \mathrm{D}^{\circ}$ & 287525 & 287550.8 & $6.38 \mathrm{e}-10$ & $6.38 \mathrm{e}-10$ \\
\hline 65 & $3 \mathrm{~s}^{2} 3 \mathrm{p}^{2}\left({ }_{2}^{3} \mathrm{P}\right){ }^{3} \mathrm{P} 4 \mathrm{p}^{4} \mathrm{P}_{1 / 2}^{\mathrm{o}}$ & 0.92 & 289025 & 289122.3 & $5.42 \mathrm{e}-10$ & $5.43 \mathrm{e}-10$ \\
\hline 66 & $3 \mathrm{~s}^{2} 3 \mathrm{p}^{2}\left({ }_{2}^{3} \mathrm{P}\right){ }^{3} \mathrm{P} 4 \mathrm{p}^{4} \mathrm{P}_{3 / 2}^{\mathrm{o}}$ & $0.80+0.063 \mathrm{~s}^{2} 3 \mathrm{p}^{2}\left({ }_{2}^{3} \mathrm{P}\right){ }^{3} \mathrm{P} 4 \mathrm{p}^{2} \mathrm{D}^{\circ}+0.043 \mathrm{~s}^{2} 3 \mathrm{p}^{2}\left({ }_{2}^{3} \mathrm{P}\right){ }^{3} \mathrm{P} 4 \mathrm{p}^{4} \mathrm{~S}^{\circ}$ & 289132 & 289233.9 & $5.58 \mathrm{e}-10$ & $5.58 \mathrm{e}-10$ \\
\hline 67 & $3 \mathrm{~s}^{2} 3 \mathrm{p}^{2}\left({ }_{0}^{1} \mathrm{~s}\right){ }^{1} \mathrm{~S} 4 \mathrm{~s}^{2} \mathrm{~S}_{1 / 2}$ & $0.90+0.033 \mathrm{p}^{4}\left({ }_{0}^{1} \mathrm{~S}\right){ }^{1} \mathrm{~S} 4 \mathrm{~s}^{2} \mathrm{~S}$ & 289489 & & $2.27 \mathrm{e}-10$ & $2.25 \mathrm{e}-10$ \\
\hline 68 & $3 \mathrm{~s}^{2} 3 \mathrm{p}^{2}\left({ }_{2}^{3} \mathrm{P}\right){ }^{3} \mathrm{P} 4 \mathrm{p}^{4} \mathrm{P}_{5 / 2}^{\circ}$ & $0.78+0.103 \mathrm{~s}^{2} 3 \mathrm{p}^{2}\left({ }_{2}^{3} \mathrm{P}\right){ }^{3} \mathrm{P} 4 \mathrm{p}^{2} \mathrm{D}^{\circ}+0.043 \mathrm{~s}^{2} 3 \mathrm{p}^{2}\left({ }_{2}^{1} \mathrm{D}\right){ }^{1} \mathrm{D} 4 \mathrm{p}^{2} \mathrm{D}^{\circ}$ & 289727 & 289830.5 & $5.70 \mathrm{e}-10$ & $5.70 \mathrm{e}-10$ \\
\hline 69 & $3 \mathrm{~s}^{2} 3 \mathrm{p}^{2}\left({ }_{2}^{3} \mathrm{P}\right){ }^{3} \mathrm{P} 4 \mathrm{p}^{2} \mathrm{D}_{3 / 2}^{\circ}$ & $0.68+0.173 \mathrm{~s}^{2} 3 \mathrm{p}^{2}\left({ }_{2}^{1} \mathrm{D}\right){ }^{1} \mathrm{D} 4 \mathrm{p}^{2} \mathrm{D}^{\circ}+0.073 \mathrm{~s}^{2} 3 \mathrm{p}^{2}\left({ }_{2}^{3} \mathrm{P}\right){ }^{3} \mathrm{P} 4 \mathrm{p}^{4} \mathrm{P}^{\circ}$ & 290224 & 1.5 & $8.10 \mathrm{e}-10$ & $8.02 \mathrm{e}-10$ \\
\hline 70 & $3 s^{2} S 3 p^{3}\left({ }_{3}^{2} D\right){ }^{3} D 3 d^{4} F_{3 / 2}^{\circ}$ & $\left.0.70+0.153 \mathrm{~s}^{2} \mathrm{~S} 3 \mathrm{p}^{3}{ }_{1}^{2} \mathrm{P}\right){ }^{3} \mathrm{P} 3 \mathrm{~d}^{4} \mathrm{~F}^{\circ}+0.113 \mathrm{~s}^{2} 3 \mathrm{p}^{2} \mathrm{P} 3 \mathrm{~d}^{2}\left({ }_{2}^{3} \mathrm{~F}\right){ }^{4} \mathrm{~F}^{\circ}$ & 291322 & & $5.69 \mathrm{e}-09$ & $5.36 \mathrm{e}-09$ \\
\hline 71 & $3 s^{2} S 3 p^{3}\left({ }_{3}^{2} D\right){ }^{3} D 3 d^{4} F_{5 / 2}^{\circ / 2}$ & $0.69+0.143 \mathrm{~s}^{2} \mathrm{~S} 3 \mathrm{p}^{3}\left({ }_{1}^{2} \mathrm{P}\right){ }^{3} \mathrm{P} 3 \mathrm{~d}^{4} \mathrm{~F}^{\circ}+0.113 \mathrm{~s}^{2} 3 \mathrm{p}^{2} \mathrm{P} 3 \mathrm{~d}^{2}\left({ }_{2}^{3} \mathrm{~F}\right){ }^{4} \mathrm{~F}^{\circ}$ & 291519 & & $5.02 \mathrm{e}-09$ & $4.76 \mathrm{e}-09$ \\
\hline 72 & $3 \mathrm{~s}^{2} 3 \mathrm{p}^{2}\left({ }_{2}^{3} \mathrm{P}\right){ }^{3} \mathrm{P} 4 \mathrm{p}^{2} \mathrm{D}_{5 / 2}^{\circ}$ & $0.62+0.143 \mathrm{~s}^{2} 3 \mathrm{p}^{2}\left({ }_{2}^{1} \mathrm{D}\right){ }^{1} \mathrm{D} 4 \mathrm{p}^{2} \mathrm{D}^{\circ}+0.143 \mathrm{~s}^{2} 3 \mathrm{p}^{2}\left({ }_{2}^{3} \mathrm{P}\right){ }^{3} \mathrm{P} 4 \mathrm{p}^{4} \mathrm{P}^{\circ}$ & 291628 & 291663.1 & $7.95 \mathrm{e}-10$ & $7.88 \mathrm{e}-10$ \\
\hline 73 & $3 \mathrm{~s}^{2} 3 \mathrm{p}^{2}\left({ }_{2}^{3} \mathrm{P}\right){ }^{3} \mathrm{P} 4 \mathrm{p}^{4} \mathrm{~S}_{3 / 2}^{\circ}$ & $0.86+0.053 \mathrm{~s}^{2} 3 \mathrm{p}^{2}\left({ }_{2}^{3} \mathrm{P}\right){ }^{3} \mathrm{P} 4 \mathrm{p}^{4} \mathrm{P}^{\circ}+0.023 \mathrm{~s}^{2} \mathrm{~S} 3 \mathrm{p}^{3}\left({ }_{3}^{4} \mathrm{~S}\right){ }^{5} \mathrm{~S} 4 \mathrm{~s}^{4} \mathrm{~S}^{\circ}$ & 291667 & 291744 & $5.64 \mathrm{e}-10$ & $5.60 \mathrm{e}-10$ \\
\hline 74 & $3 s^{2} \mathrm{~S} 3 \mathrm{p}^{3}\left({ }_{3}^{2} \mathrm{D}\right){ }^{3} \mathrm{D} 3 \mathrm{~d}^{4} \mathrm{~F}_{7 / 2}^{\mathrm{o}}$ & $\left.0.71+0.143 \mathrm{~s}^{2} \mathrm{~S} 3 \mathrm{p}^{3}{ }_{1}^{2} \mathrm{P}\right){ }^{3} \mathrm{P} 3 \mathrm{~d}^{4} \mathrm{~F}^{\circ}+0.113 \mathrm{~s}^{2} 3 \mathrm{p}^{2} \mathrm{P} 3 \mathrm{~d}^{2}\left({ }_{2}^{3} \mathrm{~F}\right){ }^{4} \mathrm{~F}^{\circ}$ & 291804 & & $5.87 \mathrm{e}-09$ & $5.52 \mathrm{e}-09$ \\
\hline 75 & $3 s^{2} S 3 p^{3}\left({ }_{3}^{2} D\right){ }^{3} D 3 d^{4} F_{9 / 2}^{\circ}$ & $0.72+0.143 \mathrm{~s}^{2} \mathrm{~S} 3 \mathrm{p}^{3}\left({ }_{1}^{2} \mathrm{P}\right){ }^{3} \mathrm{P} 3 \mathrm{~d}^{4} \mathrm{~F}^{\circ}+0.113 \mathrm{~s}^{2} 3 \mathrm{p}^{2} \mathrm{P} 3 \mathrm{~d}^{2}\left({ }_{2}^{3} \mathrm{~F}\right){ }^{4} \mathrm{~F}^{\circ}$ & 292173 & & $6.02 \mathrm{e}-09$ & $5.66 \mathrm{e}-09$ \\
\hline 76 & $3 \mathrm{~s}^{2} 3 \mathrm{p}^{2}\left({ }_{2}^{3} \mathrm{P}\right){ }^{3} \mathrm{P} 4 \mathrm{p}^{2} \mathrm{P}_{1 / 2}^{\circ}$ & $0.75+0.143 \mathrm{~s}^{2} 3 \mathrm{p}^{2}\left({ }_{2}^{1} \mathrm{D}\right){ }^{1} \mathrm{D} 4 \mathrm{p}^{2} \mathrm{P}^{\circ}+0.023 \mathrm{~s}^{2} 3 \mathrm{p}^{2}\left({ }_{0}^{1} \mathrm{~S}\right){ }^{1} \mathrm{~S} 4 \mathrm{p}^{2} \mathrm{P}^{\circ}$ & 295743 & 295670.2 & $6.98 \mathrm{e}-10$ & $6.89 \mathrm{e}-10$ \\
\hline 77 & $3 \mathrm{~s}^{2} 3 \mathrm{p}^{2}\left({ }_{2}^{3} \mathrm{P}\right){ }^{3} \mathrm{P} 4 \mathrm{p}^{2} \mathrm{P}_{3 / 2}^{\circ}$ & $0.73+0.173 \mathrm{~s}^{2} 3 \mathrm{p}^{2}\left({ }_{2}^{1} \mathrm{D}\right){ }^{1} \mathrm{D} 4 \mathrm{p}^{2} \mathrm{P}^{\circ}$ & 295849 & 295802.2 & $7.03 e-10$ & $6.94 \mathrm{e}-10$ \\
\hline 78 & $3 \mathrm{~s}^{2} 3 \mathrm{p}^{2}\left({ }_{2}^{1} \mathrm{D}\right){ }^{1} \mathrm{D} 4 \mathrm{p}^{2} \mathrm{~F}_{5 / 2}^{\mathrm{o}}$ & $0.91+0.023 \mathrm{~s}^{2} \mathrm{~S} 3 \mathrm{p}^{2}\left({ }_{2}^{3} \mathrm{P}\right){ }^{2} \mathrm{P} 3 \mathrm{~d}^{1} \mathrm{D} 4 \mathrm{p}^{2} \mathrm{~F}^{\circ}$ & 303802 & 303665.4 & $6.34 \mathrm{e}-10$ & $6.32 \mathrm{e}-10$ \\
\hline 79 & $3 \mathrm{~s}^{2} 3 \mathrm{p}^{2}\left({ }_{2}^{1} \mathrm{D}\right){ }^{1} \mathrm{D} 4 \mathrm{p}^{2} \mathrm{~F}_{7 / 2}^{\mathrm{o} / 2}$ & $0.92+0.023 \mathrm{~s}^{2} \mathrm{~S} 3 \mathrm{p}^{2}\left({ }_{2}^{2} \mathrm{P}\right){ }^{2} \mathrm{P} 3 \mathrm{~d}^{1} \mathrm{D} 4 \mathrm{p}^{2} \mathrm{~F}^{\circ}$ & 304125 & 303989.1 & $6.29 \mathrm{e}-10$ & $6.28 \mathrm{e}-10$ \\
\hline 80 & $3 s^{2} S 3 p^{3}\left({ }_{3}^{2} D\right){ }^{3} D 3 d^{2} S_{1 / 2}^{o}$ & $83 \mathrm{~s}^{2} 3 \mathrm{p}^{2} \mathrm{P} 3 \mathrm{~d}^{2}\left({ }_{2}^{3} \mathrm{P}\right){ }^{2} \mathrm{~S}^{\circ}+0.173 \mathrm{~s}^{2} \mathrm{~S} 3 \mathrm{p}^{3}\left({ }_{3}^{2} \mathrm{D}\right){ }^{1} \mathrm{D} 3 \mathrm{~d}^{2} \mathrm{~S}^{\circ}$ & 304259 & & $2.52 \mathrm{e}-09$ & $2.40 \mathrm{e}-09$ \\
\hline 81 & $3 \mathrm{~s}^{2} 3 \mathrm{p}^{2}\left({ }_{2}^{1} \mathrm{D}\right){ }^{1} \mathrm{D} 4 \mathrm{p}^{2} \mathrm{D}_{5 / 2}^{\mathrm{1} / 2}$ & $0.71+0.183 \mathrm{~s}^{2} 3 \mathrm{p}^{2}\left({ }_{2}^{3} \mathrm{P}\right){ }^{3} \mathrm{P} 4 \mathrm{p}^{2} \mathrm{D}^{\circ}$ & 306032 & 305827.4 & $7.72 \mathrm{e}-10$ & $7.52 \mathrm{e}-10$ \\
\hline 82 & $3 \mathrm{~s}^{2} 3 \mathrm{p}^{2}\left({ }_{2}^{1} \mathrm{D}\right){ }^{1} \mathrm{D} 4 \mathrm{p}^{2} \mathrm{D}_{3 / 2}^{\circ / 2}$ & $0.72+0.173 \mathrm{~s}^{2} 3 \mathrm{p}^{2}\left({ }_{2}^{3} \mathrm{P}\right){ }^{3} \mathrm{P} 4 \mathrm{p}^{2} \mathrm{D}^{\circ}$ & 306112 & 305899.6 & $7.65 \mathrm{e}-10$ & $7.46 \mathrm{e}-10$ \\
\hline 83 & $3 s^{2} S 3 p^{3}\left({ }_{3}^{2} D\right){ }^{3} D 3 d^{4} G_{5 / 2}^{o}$ & $0.88+0.073 \mathrm{~s}^{2} 3 \mathrm{p}^{2} \mathrm{P} 3 \mathrm{~d}^{2}\left({ }_{2}^{3} \mathrm{~F}\right){ }^{4} \mathrm{G}^{\circ}$ & 306552 & & $2.17 \mathrm{e}-09$ & $2.04 \mathrm{e}-09$ \\
\hline 84 & $3 s^{2} S 3 p^{3}\left({ }_{3}^{2} D\right){ }^{3} D 3 d^{4} G_{7 / 2}^{\circ / 2}$ & $0.88+0.073 \mathrm{~s}^{2} 3 \mathrm{p}^{2} \mathrm{P} 3 \mathrm{~d}^{2}\left({ }_{2}^{2} \mathrm{~F}\right){ }^{4} \mathrm{G}^{\circ}$ & 306623 & & $2.19 \mathrm{e}-09$ & $2.06 \mathrm{e}-09$ \\
\hline 85 & $3 s^{2} S 3 p^{3}\left({ }_{3}^{2} D\right){ }^{3} D 3 d^{4} G_{9 / 2}^{o}$ & $0.88+0.073 \mathrm{~s}^{2} 3 \mathrm{p}^{2} \mathrm{P} 3 \mathrm{~d}^{2}\left({ }_{2}^{3} \mathrm{~F}\right){ }^{4} \mathrm{G}^{\circ}$ & 306732 & & $2.21 \mathrm{e}-09$ & $2.08 \mathrm{e}-09$ \\
\hline 86 & $3 \mathrm{~s}^{2} \mathrm{~S} 3 \mathrm{p}^{3}\left({ }_{3}^{2} \mathrm{D}\right){ }^{3} \mathrm{D} 3 \mathrm{~d}^{4} \mathrm{G}_{11 / 2}^{\mathrm{o}}$ & $0.88+0.073 \mathrm{~s}^{2} 3 \mathrm{p}^{2} \mathrm{P} 3 \mathrm{~d}^{2}\left({ }_{2}^{3} \mathrm{~F}\right)^{4} \mathrm{G}^{\circ}$ & 306871 & & $2.23 \mathrm{e}-09$ & $2.09 \mathrm{e}-09$ \\
\hline 87 & $3 \mathrm{~s}^{2} 3 \mathrm{p}^{2}\left({ }_{2}^{1} \mathrm{D}\right){ }^{1} \mathrm{D} 4 \mathrm{p}^{2} \mathrm{P}_{1 / 2}^{\circ}$ & $0.76+0.143 \mathrm{~s}^{2} 3 \mathrm{p}^{2}\left({ }_{2}^{3} \mathrm{P}\right){ }^{3} \mathrm{P} 4 \mathrm{p}^{2} \mathrm{P}^{\circ}$ & 312019 & & $4.92 \mathrm{e}-10$ & $4.85 \mathrm{e}-10$ \\
\hline 88 & $3 \mathrm{~s}^{2} 3 \mathrm{p}^{2}\left({ }_{2}^{1} \mathrm{D}\right){ }^{1} \mathrm{D} 4 \mathrm{p}^{2} \mathrm{P}_{3 / 2}^{\mathrm{o}}$ & $0.71+0.173 \mathrm{~s}^{2} 3 \mathrm{p}^{2}\left({ }_{2}^{3} \mathrm{P}\right){ }^{3} \mathrm{P} 4 \mathrm{p}^{2} \mathrm{P}^{\circ}$ & 312497 & & $4.95 \mathrm{e}-10$ & $4.88 \mathrm{e}-10$ \\
\hline 89 & $3 s^{2} \mathrm{~S} 3 \mathrm{p}^{3}\left({ }_{3}^{2} \mathrm{D}\right){ }^{3} \mathrm{D} 3 \mathrm{~d}^{2} \mathrm{G}_{7 / 2}^{\circ}$ & $0.80+0.153 \mathrm{~s}^{2} 3 \mathrm{p}^{2} \mathrm{P} 3 \mathrm{~d}^{2}\left({ }_{2}^{1} \mathrm{G}\right)^{2} \mathrm{G}^{\circ}$ & 317986 & & $5.06 \mathrm{e}-09$ & $4.74 \mathrm{e}-09$ \\
\hline 90 & $3 s^{2} S 3 p^{3}\left({ }_{3}^{2} D\right){ }^{3} D 3 d^{2} G_{9 / 2}^{o}$ & $0.80+0.143 \mathrm{~s}^{2} 3 \mathrm{p}^{2} \mathrm{P} 3 \mathrm{~d}^{2}\left({ }_{2}^{1} \mathrm{G}\right)^{2} \mathrm{G}^{\circ}$ & 318047 & & $5.29 \mathrm{e}-09$ & $4.93 e-09$ \\
\hline 91 & $3 \mathrm{~s}^{2} \mathrm{~S} 3 \mathrm{p}^{3}\left({ }_{1}^{2} \mathrm{P}\right){ }^{3} \mathrm{P} 3 \mathrm{~d}^{4} \mathrm{D}_{1 / 2}^{\mathrm{g} / 2}$ & $0.37+0.303 \mathrm{~s}^{2} \mathrm{~S} 3 \mathrm{p}^{3}\left({ }_{3}^{2} \mathrm{D}\right){ }^{3} \mathrm{D} 3 \mathrm{~d}^{4} \mathrm{D}^{\circ}+0.183 \mathrm{~s}^{2} \mathrm{~S} 3 \mathrm{p}^{3}\left({ }_{3}^{4} \mathrm{~S}\right){ }^{5} \mathrm{~S} 3 \mathrm{~d}^{4} \mathrm{D}^{\circ}$ & 324720 & & $3.76 \mathrm{e}-10$ & $3.72 \mathrm{e}-10$ \\
\hline 92 & $3 \mathrm{~s}^{2} \mathrm{~S} 3 \mathrm{p}^{3}\left({ }_{1}^{2} \mathrm{P}\right){ }^{3} \mathrm{P} 3 \mathrm{~d}^{4} \mathrm{D}_{3 / 2}^{\circ}$ & $0.35+0.293 \mathrm{~s}^{2} \mathrm{~S} 3 \mathrm{p}^{3}\left({ }_{3}^{2} \mathrm{D}\right){ }^{3} \mathrm{D} 3 \mathrm{~d}^{4} \mathrm{D}^{\circ}+0.183 \mathrm{~s}^{2} \mathrm{~S} 3 \mathrm{p}^{3}\left({ }_{3}^{4} \mathrm{~S}\right){ }^{5} \mathrm{~S} 3 \mathrm{~d}^{4} \mathrm{D}^{\circ}$ & 324958 & & $3.72 \mathrm{e}-10$ & $3.68 \mathrm{e}-10$ \\
\hline 93 & $3 \mathrm{~s}^{2} \mathrm{~S} 3 \mathrm{p}^{3}\left({ }_{1}^{2} \mathrm{P}\right){ }^{3} \mathrm{P} 3 \mathrm{~d}^{4} \mathrm{D}_{5 / 2}^{\circ}$ & $0.33+0.283 \mathrm{~s}^{2} \mathrm{~S} 3 \mathrm{p}^{3}\left({ }_{3}^{2} \mathrm{D}\right){ }^{3} \mathrm{D} 3 \mathrm{~d}^{4} \mathrm{D}^{\circ}+0.173 \mathrm{~s}^{2} \mathrm{~S} 3 \mathrm{p}^{3}\left({ }_{3}^{4} \mathrm{~S}\right){ }^{5} \mathrm{~S} 3 \mathrm{~d}^{4} \mathrm{D}^{\circ}$ & 325417 & & $3.66 \mathrm{e}-10$ & $3.61 \mathrm{e}-10$ \\
\hline 94 & $3 \mathrm{~s}^{2} \mathrm{~S} 3 \mathrm{p}^{3}\left({ }_{1}^{2} \mathrm{P}\right){ }^{3} \mathrm{P} 3 \mathrm{~d}^{4} \mathrm{P}_{1 / 2}^{\mathrm{o} / 2}$ & $0.73+0.123 \mathrm{~s}^{2} 3 \mathrm{p}^{2} \mathrm{P} 3 \mathrm{~d}^{2}\left({ }_{2}^{3} \mathrm{P}\right){ }^{4} \mathrm{P}^{\circ}+0.113 \mathrm{~s}^{2} \mathrm{~S} 3 \mathrm{p}^{3}\left({ }_{3}^{2} \mathrm{D}\right){ }^{3} \mathrm{D} 3 \mathrm{~d}^{4} \mathrm{P}^{\circ}$ & 326067 & & $7.89 \mathrm{e}-10$ & $7.72 \mathrm{e}-10$ \\
\hline 95 & $3 \mathrm{~s}^{2} \mathrm{~S} 3 \mathrm{p}^{3}\left({ }_{1}^{2} \mathrm{P}\right){ }^{3} \mathrm{P} 3 \mathrm{~d}^{4} \mathrm{D}_{7 / 2}^{\circ}$ & $0.36+0.303 \mathrm{~s}^{2} \mathrm{~S} 3 \mathrm{p}^{3}\left({ }_{3}^{2} \mathrm{D}\right){ }^{3} \mathrm{D} 3 \mathrm{~d}^{4} \mathrm{D}^{\circ}+0.193 \mathrm{~s}^{2} \mathrm{~S} 3 \mathrm{p}^{3}\left({ }_{3}^{4} \mathrm{~S}\right){ }^{5} \mathrm{~S} 3 \mathrm{~d}^{4} \mathrm{D}^{\circ}$ & 326249 & & $3.40 \mathrm{e}-10$ & $3.36 \mathrm{e}-10$ \\
\hline 96 & $3 \mathrm{~s}^{2} \mathrm{~S} 3 \mathrm{p}^{3}\left({ }_{1}^{2} \mathrm{P}\right){ }^{3} \mathrm{P} 3 \mathrm{~d}^{4} \mathrm{P}_{3 / 2}^{\circ}$ & $0.69+0.113 \mathrm{~s}^{2} 3 \mathrm{p}^{2} \mathrm{P} 3 \mathrm{~d}^{2}\left({ }_{2}^{3} \mathrm{P}\right){ }^{4} \mathrm{P}^{\circ}+0.083 \mathrm{~s}^{2} \mathrm{~S} 3 \mathrm{p}^{3}\left({ }_{3}^{2} \mathrm{D}\right){ }^{3} \mathrm{D} 3 \mathrm{~d}^{4} \mathrm{P}^{\circ}$ & 326277 & & $5.84 \mathrm{e}-10$ & $5.73 e-10$ \\
\hline 97 & $3 \mathrm{~s}^{2} \mathrm{~S} 3 \mathrm{p}^{3}\left({ }_{1}^{2} \mathrm{P}\right){ }^{3} \mathrm{P} 3 \mathrm{~d}^{4} \mathrm{P}_{5 / 2}^{\mathrm{o}}$ & $\left.0.48+0.143 \mathrm{~s}^{2} \mathrm{~S} 3 \mathrm{p}^{3}{ }_{1}^{2} \mathrm{P}\right){ }^{3} \mathrm{P} 3 \mathrm{~d}^{2} \mathrm{D}^{\circ}+0.083 \mathrm{~s}^{2} 3 \mathrm{p}^{2} \mathrm{P} 3 \mathrm{~d}^{2}\left({ }_{2}^{3} \mathrm{P}\right){ }^{4} \mathrm{P}^{\circ}$ & 326678 & & $5.25 \mathrm{e}-10$ & $5.16 \mathrm{e}-10$ \\
\hline 98 & $3 \mathrm{~s}^{2} \mathrm{~S} 3 \mathrm{p}^{3}\left({ }_{1}^{1} \mathrm{P}\right){ }^{3} \mathrm{P} 3 \mathrm{~d}^{2} \mathrm{D}_{3 / 2}^{\circ}$ & $0.41+0.133 \mathrm{~s}^{2} 3 \mathrm{p}^{2} \mathrm{P} 3 \mathrm{~d}^{2}\left({ }_{2}^{3} \mathrm{~F}\right)^{2} \mathrm{D}^{\circ}+0.113 \mathrm{~s}^{2} \mathrm{~S} 3 \mathrm{p}^{3}\left({ }_{3}^{2} \mathrm{D}\right){ }^{1} \mathrm{D} 3 \mathrm{~d}^{2} \mathrm{D}^{\circ}$ & 326881 & & $7.97 \mathrm{e}-10$ & $7.78 \mathrm{e}-10$ \\
\hline 99 & $3 s^{2} 3 p^{2}\left({ }_{0}^{1} S\right){ }^{1} S 4 p^{2} P_{1 / 2}^{o}$ & $0.87+0.033 \mathrm{p}^{4}\left({ }_{0}^{1} \mathrm{~S}\right){ }^{1} \mathrm{~S} 4 \mathrm{p}^{2} \mathrm{P}^{\circ}$ & 327235 & & $6.24 \mathrm{e}-10$ & $6.21 \mathrm{e}-10$ \\
\hline 100 & $3 s^{2} 3 p^{2}\left({ }_{0}^{1} S\right){ }^{1} S 4 p^{2} P_{3 / 2}^{\circ}$ & $0.87+0.033 \mathrm{p}^{4}\left({ }_{0}^{1} \mathrm{~S}\right){ }^{1} \mathrm{~S} 4 \mathrm{p}^{2} \mathrm{P}^{\circ}+0.023 \mathrm{~s}^{2} 3 \mathrm{p}^{2}\left({ }_{2}^{1} \mathrm{D}\right){ }^{1} \mathrm{D} 4 \mathrm{p}^{2} \mathrm{P}^{\circ}$ & 327351 & & $6.18 \mathrm{e}-10$ & $6.14 \mathrm{e}-10$ \\
\hline 101 & $3 \mathrm{~s}^{2} \mathrm{~S} 3 \mathrm{p}^{3}\left({ }_{1}^{2} \mathrm{P}\right){ }^{3} \mathrm{P} 3 \mathrm{~d}^{2} \mathrm{D}_{5 / 2}^{\circ}$ & $0.30+0.283 \mathrm{~s}^{2} \mathrm{~S} 3 \mathrm{p}^{3}\left({ }_{1}^{2} \mathrm{P}\right){ }^{3} \mathrm{P} 3 \mathrm{~d}^{4} \mathrm{P}^{\circ}+0.093 \mathrm{~s}^{2} 3 \mathrm{p}^{2} \mathrm{P} 3 \mathrm{~d}^{2}\left({ }_{2}^{3} \mathrm{~F}\right){ }^{2} \mathrm{D}^{\circ}$ & 327371 & & $5.88 \mathrm{e}-10$ & $5.76 \mathrm{e}-10$ \\
\hline 102 & $3 s^{2} \mathrm{~S} 3 \mathrm{p}^{3}\left({ }_{1}^{2} \mathrm{P}\right){ }^{3} \mathrm{P} 3 \mathrm{~d}^{4} \mathrm{~F}_{9 / 2}^{\mathrm{o}}$ & $0.77+0.153 \mathrm{~s}^{2} \mathrm{~S} 3 \mathrm{p}^{3}\left({ }_{3}^{2} \mathrm{D}\right){ }^{3} \mathrm{D} 3 \mathrm{~d}^{4} \mathrm{~F}^{\circ}+0.033 \mathrm{~s}^{2} 3 \mathrm{p}^{2}\left({ }_{2}^{3} \mathrm{P}\right){ }^{3} \mathrm{P} 4 \mathrm{f}^{4} \mathrm{~F}^{\circ}$ & 329302 & & $7.89 \mathrm{e}-10$ & $7.52 \mathrm{e}-10$ \\
\hline 103 & $3 s^{2} \mathrm{~S} 3 \mathrm{p}^{3}\left({ }_{1}^{2} \mathrm{P}\right){ }^{3} \mathrm{P} 3 \mathrm{~d}^{4} \mathrm{~F}_{7 / 2}^{\circ}$ & $0.76+0.163 \mathrm{~s}^{2} \mathrm{~S} 3 \mathrm{p}^{3}\left({ }_{3}^{2} \mathrm{D}\right){ }^{3} \mathrm{D} 3 \mathrm{~d}^{4} \mathrm{~F}^{\circ}+0.033 \mathrm{~s}^{2} 3 \mathrm{p}^{2}\left({ }_{2}^{3} \mathrm{P}\right){ }^{3} \mathrm{P} 4 \mathrm{f}^{4} \mathrm{~F}^{\circ}$ & 329535 & & $7.53 \mathrm{e}-10$ & $7.19 \mathrm{e}-10$ \\
\hline
\end{tabular}

